\title{
Review Article Solar Neutrinos
}

\author{
V. Antonelli, ${ }^{1}$ L. Miramonti, ${ }^{1}$ C. Peña Garay, ${ }^{2}$ and A. Serenelli ${ }^{3}$ \\ ${ }^{1}$ Dipartimento di Fisica, Universitá degli Studi di Milano and INFN Milano, Via Celoria 16, 20133 Milano, Italy \\ ${ }^{2}$ Instituto de Fisica Corpuscular, CSIC-UVEG, 46071 Valencia, Spain \\ ${ }^{3}$ Instituto de Ciencias del Espacio (CSIC-IEEC), Facultad de Ciencias, Campus UAB, 08193 Bellaterra, Spain
}

Correspondence should be addressed to V. Antonelli; vito.antonelli@mi.infn.it

Received 13 July 2012; Accepted 9 October 2012

Academic Editor: Arthur B. McDonald

Copyright (C) 2013 V. Antonelli et al. This is an open access article distributed under the Creative Commons Attribution License, which permits unrestricted use, distribution, and reproduction in any medium, provided the original work is properly cited.

\begin{abstract}
The study of solar neutrinos has given a fundamental contribution both to astroparticle and to elementary particle physics, offering an ideal test of solar models and offering at the same time relevant indications on the fundamental interactions among particles. After reviewing the striking results of the last two decades, which were determinant to solve the long standing solar neutrino puzzle and refine the Standard Solar Model, we focus our attention on the more recent results in this field and on the experiments presently running or planned for the near future. The main focus at the moment is to improve the knowledge of the mass and mixing pattern and especially to study in detail the lowest energy part of the spectrum, which represents most of the solar neutrino spectrum but is still a partially unexplored realm. We discuss this research project and the way in which present and future experiments could contribute to make the theoretical framework more complete and stable, understanding the origin of some "anomalies" that seem to emerge from the data and contributing to answer some present questions, like the exact mechanism of the vacuum to matter transition and the solution of the so-called solar metallicity problem.
\end{abstract}

\section{Motivations for the Solar Neutrino Study}

The analysis of neutrinos emitted in the fusion processes inside the Sun is one of most significant examples of the relevant role played by the study of neutrino properties in elementary particle physics and astrophysics and in creating a link between these two sectors [1-13]. The pioneering work in the sixties [14-16] had the main goal of understanding better the way in which our star shines and to test solar models. But the surprising result of an apparent deficit in the electron neutrino flux reaching the detector marked the raise of the socalled solar neutrino puzzle and opened a whole new field of research, that has been central in elementary particle physics for many decades.

The experimental results obtained using different techniques in more than thirty years [17] and the parallel theoretical advancements confirmed at the end the validity of Pontecorvo's revolutionary idea of neutrino oscillation $[18,19]$, proving in a crystal clear way that neutrinos are massive and oscillating particles. This is one of the first pieces of clear evidence of the need to go beyond the standard model of electroweak interactions and the attempt to accommodate the experimental results about neutrino masses and mixing is a test that every theory "beyond the Standard Model" has to pass. Therefore, it is clear why these results had a great impact on elementary particle physics and also on cosmological models. At the same time, the possibility of measuring directly at least some components of the solar neutrino spectrum and of recovering in an indirect way the value of total solar neutrino flux have been fundamental for the progressive refinement of the standard solar model (SSM), which evolved during these years and is now in a general good agreement with the solar neutrino experiments.

Despite the fundamental steps forward made in the last decades, many questions are still open about the real nature and the main properties of neutrinos and the exact mixing mechanism, for example are neutrinos Majorana or Dirac fermions, the determination of mass hierarchy and exact mass values, accurate determination of the mixing angles, and presence of $\mathrm{CP}$ violation. The solar neutrino experiments presently running or planned for the future can contribute to solve at least some of these puzzles. The new frontier in this 
field is the study of the low energy part of the solar neutrino spectrum, which represents the great majority of the spectrum and is still an almost unexplored realm. Some of the challenges ahead are reducing significantly the indetermination on $p e p$ and $\mathrm{CNO}$ neutrinos and attaching the $p p$ solar neutrino measurement. This would be essential to test the stability and consistency of the standard explanation of the oscillation mechanism, confirming or definitely disproving the presence of discrepancies between theory and experiments, which has lately stimulated a flourishing of models introducing the so-called "Non Standard Interactions" (Section 6.1). Once more, these results would be of great interest to improve the knowledge both of elementary particle properties and interactions and of the astrophysical models of the Sun. They could help also to discriminate between different versions of the solar models, for instance, for what concerns the so-called "solar abundance problem," and to deepen the comparison with the results coming from other studies of solar properties, for example, from helioseismology. These studies would of course imply a further improvement of the already known detection techniques and the introduction of new ones (see, for instance, Section 7). Also from this point of view, solar neutrino physics will continue to give a stimulating contribution both to elementary particle physics and to astrophysics.

In the present paper we are going to treat all of these topics, focusing our attention on the important advancements of the last years, on the main open questions, and the future perspectives of solar neutrino physics. In Section 2, the interested reader can find a short review of the history of the socalled "solar neutrino puzzle," from the the radiochemical experiments results up to the first data obtained by SNO and the reactor experiment KamLAND, that solved this puzzle. The following section is devoted to the standard solar model, its main input parameters (with the relative uncertainties) and predictions, the helioseismology, and the "metallicity problem." The other ingredient essential for the calculation of the expected neutrino signal, the neutrino flavor conversion probability (in vacuum and matter), is discussed in Section 4. In Section 5, we report and discuss the important advancements which took place after 2002: from the results of the different phases of SNO and Super-Kamiokande to the discussion of the impact on solar neutrino physics of the data obtained by the reactor experiment KamLAND and finally to the first real-time measurements of the low energy solar neutrinos performed by Borexino. The discussion on the present phenomenological situation is completed in Section 6, with a particular attention to three flavors and to free fluxes analyses. We close the paper turning our attention to the near and far future, discussing the experimental and the theoretical challenges in Sections 7, 8, and 9.

\section{Brief History and Solution of the Solar Neutrino Problem}

2.1. From Homestake to Super-Kamiokande. The first experiment built to detect solar neutrinos took place in the Homestake gold mine in South Dakota [14-16]. The detector consisted of a large tank containing 615 metric tons of liquid perchloroethylene, chosen because it is rich in chlorine and the experiment operated continuously from 1970 until 1994. Neutrinos were detected via the reaction:

$$
v_{e}+{ }^{37} \mathrm{Cl} \longrightarrow{ }^{37} \mathrm{Ar}+e^{-}
$$

The energy threshold of this reaction, $E_{\mathrm{th}}=814 \mathrm{keV}$, allowed the detection of ${ }^{7} \mathrm{Be}$ and ${ }^{8} \mathrm{~B}$ (and a small signal from the $\mathrm{CNO}$ and $p e p$ ) but not that of $p p$ neutrinos, because of their low maximal energy of $0.42 \mathrm{MeV}$. The radioactive ${ }^{37} \mathrm{Ar}$ isotopes decay by the electron capture with a $\tau_{1 / 2}$ of about 35 days into ${ }^{37} \mathrm{Cl}^{*}$ :

$$
{ }^{37} \mathrm{Ar}+e^{-} \longrightarrow{ }^{37} \mathrm{Cl}^{*}+v_{e}
$$

Once a month, after bubbling helium through the tank, the ${ }^{37} \mathrm{Ar}$ atoms were extracted and counted. The number of atoms created was only about 5 atoms of ${ }^{37}$ Ar per month in 615 metric tons $\mathrm{C}_{2} \mathrm{Cl}_{4}$. The number of detected neutrinos was lower (about 1/3) than the value expected by the Solar Standard Model. This discrepancy is the essence of the solar neutrino problem, which has been for many years an important puzzle among physicists.

There were three possible explanations to the solar neutrino problem. The first one was to consider that Homestake could be wrong, that is, the Homestake detector could be inefficient and, in this case, its reactions would not have been predicted correctly. After all, to detect a handful of atoms per week in more than 600 metric tons of material is not an easy task (the science that studies the interior of the Sun by looking at its vibration modes). The second one was to consider that the SSM was not correct, but as helioseismology started to provide independent tests of solar models, the SSM passed all tests. Indeed, nonstandard solar models constructed ad hoc to resolve the solar neutrino problem seemed very unlikely when scrutinized under the light of helioseismology. The third one, and the strangest hypothesis, was to consider that something happens to the neutrinos while traveling from the core of the Sun to the Earth.

The first real-time solar neutrino detector, Kamiokande, was built in Japan in 1982-1983 [20]. It consisted of a large water Čerenkov detector with a total mass of 3048 metric tons of pure water. In real-time neutrino experiments, scientists study the bluish light produced by the electrons scattered by an impinging neutrino according to the following equation:

$$
v_{x}+e^{-} \longrightarrow v_{x}+e^{-} \text {. }
$$

In the Kamiokande detector, light is recorded by 1000 photomultiplier tubes (PMT) and the energy threshold of the reaction is $E_{\mathrm{th}}=7.5 \mathrm{MeV}$; therefore, only ${ }^{8} \mathrm{~B}$ and hep neutrinos are detected (Here and in the rest of the paper, following the convention commonly adopted in the literature, we use the term "energy threshold" to indicate the lowest observable neutrino energy also for Čerenkov's detector experiments. However, it is important to bear in mind that for these experiments, differently from the radiochemical ones, there is not a real energy threshold for the reaction and the lowest limit on 
the detectable energy is, instead, mainly due to the difficulty of separating the signal from the radioactive background due to natural sources and impurities.). At the beginning of the 90s, a much larger version of the detector was built, Super-Kamiokande, where the active mass was more than 50000 metric tons of pure water viewed by about 11200 PMTs. In Super-Kamiokande, the energy threshold was lowered to $E_{\text {th }}=5.5 \mathrm{MeV}$ [21].

Radiochemical experiments integrate in time and in energy because they are slow and need time to produce measurable results. This causes the loss of information about single individual energy values. In real-time experiments, instead, it is possible to obtain single values and therefore a spectrum energy to distinguish the different neutrino contributions. Furthermore, given that the scattered electron maintains the same direction of the impinging neutrino, it is possible to infer the direction of the incoming neutrino and therefore to point at its source. This proved that the detected neutrinos actually came from the Sun. The number of detected neutrinos was about $1 / 2$ lower than the number of expected ones, aggravating the solar neutrino problem.

Until 1990, there were no observations of the initial reaction in the nuclear fusion chain, that is, the detection of $p p$ neutrinos, which are less model dependent and hence more significant to test the hypothesis that fusion of hydrogen powers the Sun. Two radiochemical experiments were built in order to detect solar $p p$ neutrinos, both employing the reaction

$$
v_{e}+{ }^{71} \mathrm{Ga} \longrightarrow{ }^{71} \mathrm{Ge}+e^{-},
$$

which has a threshold of $E_{\mathrm{th}}=233 \mathrm{keV}$.

In the Gallex experiment, located at the Gran Sasso underground laboratory in Italy, 30 metric tons of natural gallium were employed [22, 23], while in the Soviet-American experiment (SAGE), located in the Baksan underground laboratory, there were more than 50 metric tons of metallic gallium [24]. Calibration tests with an artificial neutrino source, ${ }^{51} \mathrm{Cr}$, confirmed the efficiency of both detectors. Once again, the measured neutrino signal was smaller than predicted by the SSM $(\approx 60 \%)$.

All experiments detected fewer neutrinos than expected from the SSM. Table 1 summarizes the ratios between the observed and the expected neutrino interaction rates for all the experiments before SNO.

\subsection{The Advent of SNO and KamLAND: The Solution of} the Solar Neutrino Problem. The real breakthrough in solar neutrino physics was due to the advent of the SNO (Sudbury Neutrino Observatory) experiment. It had the peculiarity to measure simultaneously, by means of a deuterium Čerenkov detector, three different interaction channels for neutrinos: the neutral current (NC: $\left.v_{X}+d \rightarrow v_{X}+p^{+}+n\right)$, receiving contributions from all active flavors, the elastic scattering (ES: $v_{X}+e^{-} \rightarrow v_{X}+e^{-}$), and the charged current (CC: $v_{e}+d \rightarrow$ $\left.e^{-}+p^{+}+p^{+}\right)$, that is, sensitive only to electronic neutrinos. In this way, it has been possible to prove in a clear and direct way that the measured total neutrino flux was in a very good agreement with the SSM predictions, but only a fraction of
TABLE 1: Ratios of the observed versus expected neutrino rates in the four solar neutrino experiments (before SNO, see later).

\begin{tabular}{ll}
\hline Homestake & $0.34 \pm 0.03$ \\
Super-K & $0.46 \pm 0.02$ \\
SAGE & $0.59 \pm 0.06$ \\
Gallex and GNO & $0.58 \pm 0.05$ \\
\hline
\end{tabular}

these neutrinos had conserved its flavor during their way from the production point in the Sun to the detector.

The first SNO data [25], including elastic scattering and charged current analysis, published in 2001, confirmed the results obtained by previous solar neutrino experiments, mainly by Super-Kamiokande [26], providing a significant evidence (at the $3.3 \sigma$ level) of the presence of a nonelectronic active neutrino component in the solar flux. For the first time, it was possible to indicate the large mixing angle (LMA) as the preferred solution of the solar neutrino puzzle, even if different alternative possibilities (and in particular the low probability, low mass - LOW- solution) were still surviving $[27,28]$. In the following years, the SNO experiment measured also the neutral current channel, using different techniques. The data of these different "phases" of the experiment are usually reported as SNO I [29], SNO II [30] (characterized by the addition of salt to improve the efficiency of neutral current detection) and SNO III [31] (with the use of helium chamber proportional counters).

The year 2002 is very often denoted as the "annus mirabilis" of solar neutrino physics: in April the first SNO results including neutral current detection [29,32] marked a turning point in the history of the solar neutrino problem, in October the Nobel prize for physics was awarded to Davis [33] and Koshiba (for their pioneering work on the detection of cosmic neutrinos), and in December of the same year the first results of the Kamiokande Liquid scintillator antineutrino detector (KamLAND) [34] offered the first clear terrestrial confirmation of the validity of the oscillation solution to the solar neutrino problem.

The total ${ }^{8} \mathrm{~B}$ neutrino flux, $\phi_{\mathrm{NC}}=5.09_{-0.43}^{+0.44}(\text { stat })_{-0.43}^{+0.46} \times$ (syst) $\times 10^{6} \mathrm{~cm}^{-2} \mathrm{~s}^{-1}$, measured by SNO with neutral currents was in a very good agreement with the SSM [35]. Assuming the standard shape for the component of the solar neutrino flux (undistorted spectrum hypothesis), the SNO collaboration recovered also a value of the nonelectronic component of the flux which was $5.3 \sigma$ different from zero, providing a direct proof of the validity of the oscillation hypothesis. These data were also decisive to indicate the LMA region as the solution to the solar neutrino puzzle.

Looking at the oscillation probability, it is apparent that the reactor experiments that run before KamLAND, and used neutrino energy beams of the order of the $\mathrm{MeV}$ with a baseline of the order of $1 \mathrm{~km}$, could test only values of $\Delta m^{2}$ above $10^{-3} \mathrm{eV}^{2}$ (For instance, in a simple 2-flavor analysis, the flavor transition probability is given by the expression $P_{12}=\sin ^{2}\left(2 \theta_{12}\right) \sin ^{2}\left(\Delta m_{12}^{2}\left(\mathrm{eV}^{2}\right) L(\mathrm{~km}) / 4 E(\mathrm{GeV})\right)$, where $\theta_{12}$ is the mixing angle between the two flavors, $\Delta m_{i j}^{2} \equiv m_{1}^{2}-m_{2}^{2}$ the difference of the masses squared, $L$ the distance traveled, 
and $E$ the neutrino energy.). The KamLAND experiment, instead, with an average baseline of about $180 \mathrm{~km}$, was ideal to probe the LMA region, which corresponds to values of $\Delta m^{2}$ of the order $10^{-5}-10^{-4} \mathrm{eV}^{2}$ [36-38]. The KamLAND experiment studied the ratio of the number of inverse $\beta$ decay events (due to reactor $\bar{v}_{e}$ with an energy threshold of $3.4 \mathrm{MeV}$ ) to the expected number of events without disappearance and also the spectrum shape [34]. The observed deficit of events was inconsistent with the expected rate in absence of oscillation at the $99.95 \%$ confidence level.

Since one would expect a negligible reduction of the $\bar{v}_{e}$ flux from the SMA, LOW, and vacuum solar neutrino solutions, the LMA was the only oscillation solution compatible with KamLAND results and CPT invariance. This evidence was further reinforced by the data published by the collaboration in the following years (with greater statistical precisions and reduced systematic errors), which showed also a spectral distortion in a very good agreement with the oscillation solution [39-41]. KamLAND data also restricted the allowed LMA region in a significant way. The preferred values for $\Delta m_{12}^{2}$ and $\theta_{12}$ are slightly higher than the ones corresponding to the best fit solution of the solar neutrino experiments, but this small tension can be explained by taking into account the experimental uncertainties. Moreover, the difference on the $\Delta m_{12}^{2}$ parameter has been reduced by the more recent solar neutrino data.

\section{Standard Solar Model}

SSMs have to be understood, primarily, as a framework within which solar models can be constructed and clear predictions can be made with respect to the properties of the solar interior, including the production of solar neutrinos. The defining characteristics are simple: the SSM is the result of the evolution of a $1 \mathrm{M}_{\odot}$ star since its formation and the evolutionary models have to include the physical ingredients considered standard in stellar structure and evolution models (here, standard also implies trying to keep to a minimum the number of free tunable parameters-knobs-in the model). SSMs are therefore progressively refined as our understanding of stellar physics progresses.

In practice, an SSM is constructed as follows. An initial chemically homogeneous model of a $1 \mathrm{M}_{\odot}$ stellar model on the premain sequence is constructed with a composition determined by a guess (educated one) for the initial mass fractions of hydrogen $X_{\text {ini }}$, helium $Y_{\text {ini }}$, and metals $Z_{\text {ini }}\left(X_{\text {ini }}+\right.$ $\left.Y_{\text {ini }}+Z_{\text {ini }}=1\right)$; additionally, a third free parameter has to be specified, the mixing length parameter $\alpha_{\mathrm{MLT}}$ of convection. This model is then evolved up to the solar system age $\tau_{\odot}=$ $4.57 \mathrm{Gyr}[42,43]$. At this age, the model is required to match the present-day solar luminosity $L_{\odot}$ and radius $R_{\odot}$, as well as the surface metal-to-hydrogen abundance ratio $(Z / X)_{\odot}$. The initial and final surface metal-to-hydrogen ratios differ by about $10 \%$ to $15 \%$ due to the effects of gravitational settling. In general, the SSM constructed with the first set of guesses for $\alpha_{\mathrm{MLT}}, Y_{\text {ini }}$, and $Z_{\text {ini }}$ will not lead to a satisfactory agreement with the surface constraints, and an iterative procedure is used to refine the free parameters until the right surface conditions are achieved at $\tau_{\odot}$. In general, surface conditions are matched to one part in $10^{5}$ or $10^{6}$ within two or three iterations. It is important to keep in mind that the SSM is not just a snapshot aimed at representing the present-day structure of the Sun, but actually the result of taking into account all its previous history. There are alternative ways to construct a model of the present-day solar structure using, for example, helioseismic constraints. This kind of models is constructed "ad-hoc" to match helioseismic data and is, therefore, a limited predictive power.

The internal structure of an SSM depends on the values adopted for the three constraints mentioned above and, of course, on the physical inputs of the models such as the radiative opacities, cross-sections of nuclear reactions, and others. Next, we describe the changes/updates that have occurred during the last decade that impact predictions of solar models.

\subsection{Input Physics and Parameters}

3.1.1. Solar Surface Composition. The constraint imposed by the surface metallicity of the Sun or, more precisely, the surface metal-to-hydrogen ratio $(Z / X)_{\odot}$ is critical in the construction of solar models. The reason is that, aside from the 10 to $15 \%$ change in this value due to the action of gravitational settling, $(Z / X)_{\odot}$ determines almost directly the metallicity of solar models. As for any other star, the metal content in the Sun has a fundamental role in its structure through its contribution to the radiative opacity $\kappa$, which determines, in turn, the temperature gradient in the radiative solar interior. It is important, in fact, that the abundance of individual metals are accurately determined, because different elements contribute to the radiative opacities in different regions of the Sun.

The abundance of metals in the solar surface has to be determined or inferred from a variety of sources: photospheric abundances from solar spectra, chemical analysis of primitive meteorites, emission lines from the solar corona, and composition of the solar wind [44]. While meteoritic abundances are the most precisely determined, at $2 / 3$ of the solar metallicity is composed by the volatile elements $\mathrm{C}, \mathrm{N}$, and $\mathrm{O}$ and can only be determined from the analysis of the solar spectrum.

Over the last decade, the development of three-dimensional radiation hydrodynamic (3D RHD) models of the solar atmosphere has prompted a thorough revision of the solar composition determined from the solar spectrum. These 3D RHD models of the solar atmosphere capture the dynamics of convection and its interaction with the radiation field and are able to reproduce features such as the solar granulation pattern, observed limb-darkening, and asymmetries in the shapes of spectral lines [45]. The structure of the solar model atmospheres derived by different groups are nicely consistent with each other, adding to the credibility of the models. Newly derived spectroscopic abundances rely on the $3 \mathrm{D}$ atmosphere model or more appropriately on a one-dimensional model obtained from a suitably averaged 3D model, as the background on top of which detailed radiative transfer and line formation calculations are performed a posteriori. It is this 
second step that leads, finally, to the determination of the abundances of the different elements. The most thorough and consistent determination of the solar photospheric abundances based on 3D model atmospheres has been presented by Asplund and collaborators [46, 47], although the revision on key elements like oxygen was initially published already in 2001 [48]. In addition to using 3D RHD atmosphere models, nonlocal thermodynamic equilibrium has been taken into account when computing the line formation for some key elements such as $\mathrm{C}, \mathrm{N}$, and O. Also, and this is of particular importance for oxygen, blends in the solar spectrum that had been previously unnoticed were identified and taken into account in the determination of abundances. The most relevant result in the context of solar models and neutrinos is that abundances of CNO elements (also Ne, but this is mostly because its abundance ratio to oxygen is assumed fixed) have been revised down by $30 \%$ to $40 \%$. Combining the abundance of all metals, the present-day metal-to-hydrogen ratio that has been obtained is $(Z / X)_{\odot}=0.0178$ [46]. This represents a large decrease in comparison with previously accepted values, 0.0245 [49] and 0.0229 [50], that have been widely used in solar modeling. We note, however, that results by Asplund have not been unchallenged. In fact, also based on 3D RHD model solar atmospheres, larger $\mathrm{CNO}$ abundances have been derived [51] to yield $(Z / X)_{\odot}=0.0209$, much closer to older determinations. Discrepancies between authors seem to have their origin at the preferred set of spectral lines each group uses and on using either a spectral synthesis or equivalent width techniques to determine the final abundances.

In the last decade, there have been two flavors in SSM calculations. In one case, a high solar metallicity from older determinations $[49,50]$ is adopted; we will generically refer to these models as high- $Z$ solar models. In the other case, a low $(Z / X)_{\odot}[46,47]$ is taken from and we refer to these, not surprisingly, as the low-Z solar models. Differences in the structure of high $-Z$ and low $-Z$ models are readily noticeable in quantities such as the internal sound speed and density profiles, the depth of the solar convective envelope, and the surface helium abundance among others. The deficit that low$Z$ models have in matching helioseismic constraints has been named the solar abundance problem in the literature, in clear analogy to the solar neutrino problem. We discuss it in some detail in Section 3.2.

3.1.2. Radiative Opacities. The most widely used calculations of atomic radiative opacities, appropriate for solar interiors, are those from OPAL [52]. However, the opacity project (OP) released in 2005 a completely independent set of atomic radiative opacities for stellar interiors [53]. In the case of the solar radiative interior, differences between OPAL and OP Rosseland mean opacities are of the order of a few percent, with OP being larger by about $3 \%$ at the base of the convective zone and $1 \%$ to $2 \%$ smaller in the central regions (see Figure 7 in [53]). At low temperatures neither OP nor OPAL atomic opacities are adequate, because the possible formation of molecules have to be taken into account and, therefore, they have to be complemented by low-temperature opacities [54]. However, due to the relatively high solar temperature, their influence in the properties of solar models is rather limited.
3.1.3. Nuclear Reactions Cross-Sections. Experimental and theoretical work on the determination of nuclear crosssections have been very active fields with a strong impact on solar model predictions of solar neutrino fluxes (A nonresonant charged-particle induced reaction cross-section can be written as $\sigma(E)=(S(E) / E) \exp [-2 \pi \eta(E)]$ where $\eta(E)=$ $Z_{1} Z_{2} \alpha / v$ is the Sommerfeld parameters, $v=\sqrt{(2 E / \mu), \alpha \text { the }}$ fine structure constant in natural units, and $\mu$ the reduced mass of the interacting nuclei. The nuclear physics is isolated in $S(E)$, the astrophysical or $S$-factor, a slowly varying function of energy that can be more accurately extrapolated from experimental data down to the energy of the Gamow peak.). Recently, a set of recommended rates and uncertainties, expressed through the $S$-factor, for all the reactions both in the pp-chains and CNO-bicycle that are relevant to solar modeling and neutrino production, has been published (Solar Fusion II, [55], hereafter SFII). The results presented in SFII reflect the progress made in laboratory and theoretical nuclear astrophysics over the last decade, since the publication of the seminal Solar Fusion I (SFI) article [56]. Unfortunately, for reasons of space, here we cannot review in detail every reaction. Instead, we provide in Table 2 the standard $S$-factors at zero energy, $S(0)$, and the uncertainties recommended in SFII for the most relevant reactions. For comparison, with results from SFI are also shown. The impact of changes in key reactions on the production of neutrino fluxes is discussed in Section 3.3. The reader is referred to the SFII paper and references therein for details on the experimental and theoretical developments in nuclear astrophysics related to the Sun during the last decade.

3.2. Solar Models: Helioseismology. Helioseismology, the study of the natural oscillations of the Sun, provides a unique tool to determine the structure of the solar interior. The $90 \mathrm{~s}$ witnessed a rapid development of helioseismic observations and analysis techniques, which led, in very few years, to an accurate characterization of the solar interior [57]. The agreement between SSMs and helioseismic inferences of the solar structure $[35,58]$ provided a strong support to the accuracy with which SSMs could predict the ${ }^{8} \mathrm{~B}$ neutrino flux and, therefore, a strong indication, before Kamland and SNO results found evidence of neutrino flavor oscillations, that the solution to the solar neutrino problem had to be found in the realm of particle physics.

In the context of the present paper, the most relevant results from helioseismology are the following (The Sun is characterized by an outer region where energy is transported by convection. The boundary between this region, located at $R_{\mathrm{CZ}}$, and the radiative interior can be accurately located by helioseismology because the discontinuity in the slope of the temperature gradient across this boundary leaves its imprint in the solar sound speed profile. The depth of the envelope can be located by helioseismology because properties of solar oscillations are sensitive to the derivative of the sound speed as a function of depth.). The depth of the convective envelope is $R_{\mathrm{C} Z}=0.713 \pm 0.001 R_{\odot}$ [59] and the surface helium abundance $Y_{S}=0.2485 \pm 0.0034$ [60]. The sound speed differences between the Sun and a reference solar model can 
TABLE 2: Standard astrophysical factors and uncertainties for key nuclear reactions in the $p p$-chains and CNO-bicycle. SFII represents the state-of-the-art [55]; SFI [56] shows, for comparison, the situation around 1998.

\begin{tabular}{lccc}
\hline & Reaction & SFII & SFI \\
& & $S(0)[\mathrm{keV} \mathrm{b}]$ & $S(0)[\mathrm{keV} \mathrm{b}]$ \\
\hline $\mathrm{S}_{11}$ & $p\left(p, e^{+} v_{e}\right) d$ & $4.01 \times 10^{-22}(1 \pm 0.010)$ & $4.00 \times 10^{-22}(1 \pm 0.005)$ \\
$\mathrm{S}_{33}$ & ${ }^{3} \mathrm{He}\left({ }^{3} \mathrm{He}, 2 p\right)^{4} \mathrm{He}$ & $5.21 \times 10^{3}(1 \pm 0.052)$ & $5.4 \times 10^{3}(1 \pm 0.074)$ \\
$\mathrm{S}_{34}$ & ${ }^{3} \mathrm{He}\left({ }^{4} \mathrm{He}, \gamma\right)^{7} \mathrm{Be}$ & $5.6 \times 10^{-1}(1 \pm 0.054)$ & $5.3 \times 10^{-1}(1 \pm 0.094)$ \\
$\mathrm{S}_{\text {hep }}$ & ${ }^{3} \mathrm{He}\left(p, e^{+} v_{e}\right)^{4} \mathrm{He}$ & $8.6 \times 10^{-20}(1 \pm 0.30)$ & $2.3 \times 10^{-20}$ \\
$\mathrm{~S}_{17}$ & ${ }^{7} \mathrm{Be}(p, \gamma)^{8} \mathrm{~B}$ & $2.08 \times 10^{-2}(1 \pm 0.077)$ & $1.9 \times 10^{-2}\left(1_{-0.10}^{+0.20}\right)$ \\
$\mathrm{S}_{1,14}$ & ${ }^{14} \mathrm{~N}(p, \gamma)^{15} \mathrm{O}$ & $1.66(1 \pm 0.072)$ & $3.5\left(1_{-0.46}^{+0.11}\right)$ \\
\hline
\end{tabular}

be obtained by inversion from the oscillation frequencies with a formal error of a few parts per $10^{-4}$ for most of the solar interior $0.07 \lesssim R / R_{\odot} \lesssim 0.95[61,62]$. Most recently, using a time series 4752 days long from the Birmingham Solar Oscillation Network, improved results on the sound speed in the solar core have been obtained [63]. The density profile can also be determined from the inversion of frequencies, but with worse precision than for the sound speed, and we therefore assign to it a secondary role in constraining the solar structure.

As mentioned previously, metals determine to a large extent the radiative opacity in the solar interior and, in this way, define the temperature stratification from below of the convective envelope inwards, to the solar center. At the base of the convective zone, for example, metals are responsible for about $70 \%$ of the total radiative opacity with $\mathrm{O}, \mathrm{Fe}$, and $\mathrm{Ne}$ being the main contributors. In the solar core, where light metals are completely ionized, the contribution from $\mathrm{Fe}$ and, to a lesser extent $\mathrm{Ni}, \mathrm{Si}$, and $\mathrm{S}$, is still above $30 \%$. In view of this, it is not surprising that the low $\mathrm{CNO}$ and $\mathrm{Ne}$ abundances determined from 3D model atmospheres have a strong impact on the structure of the solar interior.

It has been clear since initial works where low-Z SSMs were presented that low $(Z / X)_{\odot}$ values posed a problem, later named the solar abundance problem, for solar modeling [60, 67-69]. In short, all helioseismic predictions of these models are in disagreement with observations. On the other hand, high-Z SSMs have consistently reproduced earlier success [58]. The solar abundance problem represents the incompatibility between the best solar atmosphere and interior models available [70]. In this paper, we will base the presentation and discussion of results on the most up-to-date standard solar models that we identify as SFII-GS98 and SFII-AGSS09 [64], representative of high-Z and low-Z SSM families defined in Section 3.1, respectively. With the exception made on small quantitative variations, results based on these models are extensible to results for all SSMs available in the literature corresponding to each of the two families.

The most important characteristics of the SFII-GS98 and SFII-AGSS09 models are summarized in Table 3. Helioseismic constraints are also included for comparison when appropriate. The disagreement between SFII-AGSS09 and helioseismic data is evident in the surface metallicity and helium abundances, $Z_{S}$ and $Y_{S}$, and in the depth of the convective envelope $R_{\mathrm{CZ}}$. A similar conclusion could be drawn from comparing $Y_{\text {ini }}$; however, the determination of the solar value of $Y_{\text {ini }}$ [71] depends strongly on the seismic value for $Y_{S}$ and, therefore, it is not an independent constraint. When model uncertainties are included, the discrepancy between SFII-AGSS09 and seismic results are, for each of the quantities mentioned above, of the order 3 to $4-\sigma$ [72]. On the contrary, the SFII-GS98 model performs very well, within $1-\sigma$, when model uncertainties are accounted for.

Very explicit manifestations of the solar abundance problem are shown in the plots in Figure 1, where degradation in the sound speed and density profiles found in low-Z SSMs are clearly evident. Particularly the peak in the sound speed profile differences found right below the convective zone is 4 times larger in the low- $Z$ SFII-AGSS09 than in the high- $Z$ SFII-GS98 model. The reason is the wrong location of $R_{\mathrm{CZ}}$ in the model, caused by the lower opacity which, in turn, is due to the low abundance of metals. The density profile also shows very large discrepancies, but they are less telling. Density inversions include as a constraint the known value of the solar mass and for this reason small differences in the core, where density is large, translate into the large difference seen in the outer envelope. The average rms in the sound speed and density differences, $\langle\delta c / c\rangle$ and $\langle\delta \rho / \rho\rangle$, also show that low-Z models are about 4 times worse than high $-Z$ models.

Low-degree helioseismology provides useful information about the solar innermost regions. Specific combinations of mode frequencies enhance the signal that the structure of the solar core imprints on the oscillation pattern [73]. This has been used to determine the mean molecular weight averaged over the innermost $20 \%$ solar core [66], $\left\langle\mu_{C}\right\rangle$ in Table 3 . Comparison with SSMs results shows that $\left\langle\mu_{C}\right\rangle$ is too low in low$Z$ models as a result of the lower helium abundance $\left(Y_{C}\right)$. This is due to the lower temperature in the solar core and the constraint imposed by the solar luminosity. The decreased nuclear energy production originated by a smaller core temperature has to be compensated by an increased hydrogen mass fraction, therefore leading to a lower molecular weight. It is interesting to note this puts a stringent constraint in the amount of rotational mixing that can take place in the solar core if the low- $Z$ abundances are correct, since any mixing would lower the molecular weight even more, by bringing fresh hydrogen from outer regions, and make the agreement with helioseismic data worse.

The current situation regarding SSMs and their performance against helioseismic inferences on the solar structure can be summarized as follows. SSMs that use solar abundances derived from $1 \mathrm{D}$ model atmospheres $[49,50]$, that 
TABLE 3: Main characteristics of SSMs representative of high-Z (GS98) and low-Z (AGSS09) solar compositions. Models have been computed including the most up-to-date input physics [64]. Helioseismic constraints are given when available. See text for details.

\begin{tabular}{|c|c|c|c|}
\hline & SFII-GS98 & SFII-AGSS09 & Helioseismology \\
\hline$\overline{(Z / X)_{\odot}}$ & 0.0229 & 0.0178 & - \\
\hline$Z_{S}$ & 0.0170 & 0.0134 & $0.0172 \pm 0.002[65]$ \\
\hline$Y_{S}$ & 0.2429 & 0.2319 & $0.2485 \pm 0.0034[60]$ \\
\hline$R_{\mathrm{CZ}} / R_{\odot}$ & 0.7124 & 0.7231 & $0.713 \pm 0.001[59]$ \\
\hline$\langle\delta c / c\rangle$ & 0.0009 & 0.0037 & - \\
\hline$\langle\delta \rho / \rho\rangle$ & 0.011 & 0.040 & - \\
\hline$Z_{C}$ & 0.0200 & 0.0159 & - \\
\hline$Y_{C}$ & 0.6333 & 0.6222 & - \\
\hline$\left\langle\mu_{C}\right\rangle$ & 0.7200 & 0.7136 & $0.7225 \pm 0.0014[66]$ \\
\hline$Z_{\text {ini }}$ & 0.0187 & 0.0149 & - \\
\hline$Y_{\text {ini }}$ & 0.2724 & 0.2620 & 一 \\
\hline
\end{tabular}
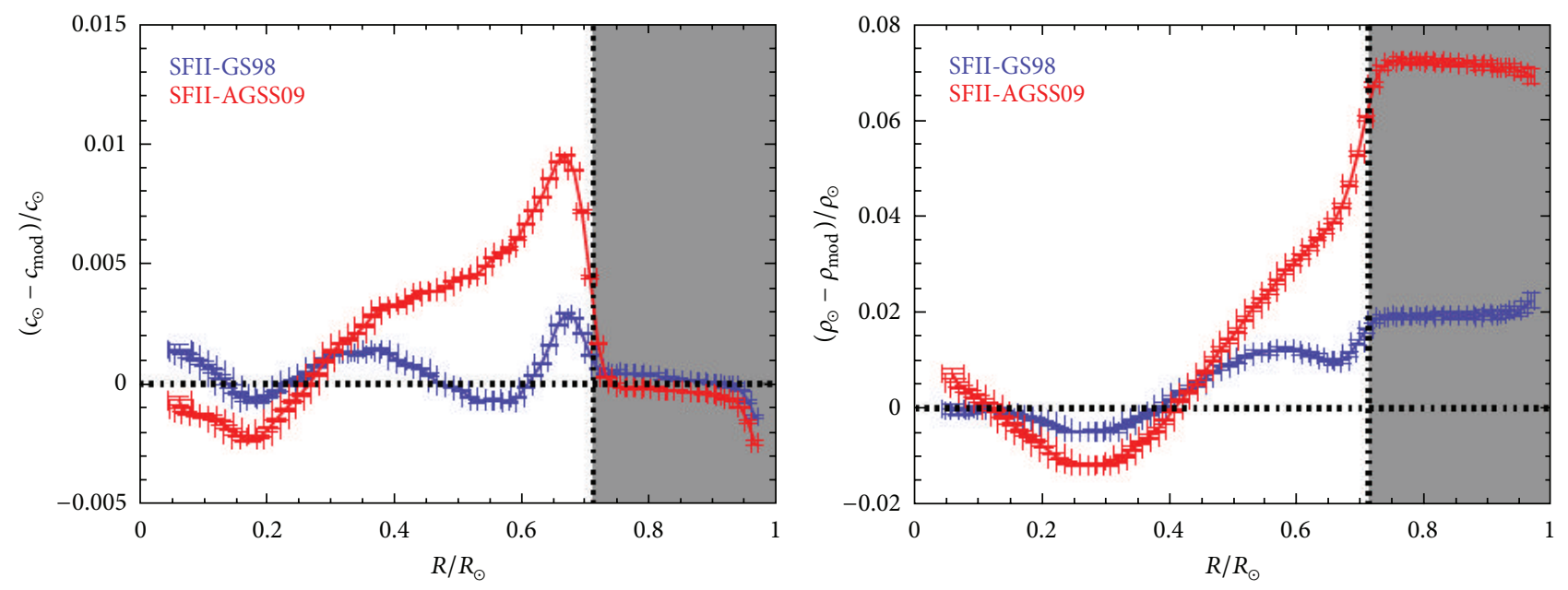

FIGURE 1: Sound speed and density relative differences between solar models and the Sun as determined from helioseismic inversions [72]. The convective envelope is depicted by the grey area.

is, high- $Z$ models, reproduce overall the most important seismic constraints. Improvements in the input physics, for example, radiative opacities and nuclear reaction rates, that have occurred over the last 10 years introduce only small changes to the solar structure as seen by helioseismology. On the other hand, the solar abundance problem arises if the solar surface composition used to construct SSMs are derived from the most sophisticated 3D RHD solar model atmospheres. The family of low-Z SSMs does not match any helioseismic constraint.

Have we reached the limit where the paradigm of the SSM is not good enough as a model of the solar interior? Are the 3D-based determinations of solar abundances systematically underestimating the metallicity of the solar surface? Does the microscopic input physics in solar models, for example, radiative opacities, need to be thoroughly revised? It is not possible to advance answers to these questions, but solar neutrino experiments can play an important role in guiding the research towards the solution of the solar abundance problem. In the next section, we discuss the current status on the theoretical predictions of solar neutrino fluxes and the prospects of using solar neutrinos to constraint the properties of the solar core.

\subsection{Solar Models: Neutrino Fluxes}

3.3.1. Production. Based on theoretical arguments and indirect evidence, it has long been believed that the source of energy of the Sun is the conversion of protons into helium, $4 \mathrm{p} \rightarrow{ }^{4} \mathrm{He}+2 e^{+}+2 v_{e}+\gamma$. The original quest for solar neutrinos was indeed the search for the experimental confirmation of this hypothesis (Under peculiar conditions reached in advanced phases of stellar evolution, hydrogen can be converted into helium by other cycles like the NaMg-cycle. While important for nucleosynthesis or intermediate mass elements, these processes are not energetically relevant.). In more detail, hydrogen burning in the Sun (and in all other hydrogen-burning stars) takes place either through the ppchains or the CNO-bicycle $[43,75]$. Proton fusion through the $\mathrm{pp}$-chains is a primary process because only protons need to be present in the star. On the contrary, the CNO-bicycle is secondary because the proton fusion relies on, and is regulated by, the abundance of $\mathrm{C}, \mathrm{N}$, and $\mathrm{O}$ which act as catalyzers. This qualitative difference is very important, since it renders neutrino fluxes from the CNO-bicyle a very good diagnostic tool to study properties of the solar core, particularly its composition, as it will be discussed below. A general discussion 
TABLE 4: SSM predictions for solar neutrino fluxes (second and third columns) and solar neutrino fluxes (fourth column) inferred from all available neutrino data. Units are, in $\mathrm{cm}^{-2} \mathrm{~s}^{-1}$, as usual: $10^{10}(p p), 10^{9}\left({ }^{7} \mathrm{Be}\right), 10^{8}\left(p e p,{ }^{13} \mathrm{~N},{ }^{15} \mathrm{O}\right) 10^{6}\left({ }^{8} \mathrm{~B}\right.$, $\left.{ }^{17} \mathrm{~F}\right)$, and $10^{3}($ hep). Note that the limit on the fluxes relative to the $\mathrm{CNO}$ cycle do not contain yet the information coming from recent Borexino result [74]. A revised analysis including these data is in progress.

\begin{tabular}{lcccc}
\hline Flux & SFII-GS98 & SFII-AGSS09 & Solar & BP04 \\
\hline$p p$ & $5.98(1 \pm 0.006)$ & $6.03(1 \pm 0.006)$ & $6.05\left(1_{-0.011}^{+0.003}\right)$ & $5.94(1 \pm 0.01)$ \\
$p e p$ & $1.44(1 \pm 0.012)$ & $1.47(1 \pm 0.012)$ & $1.46\left(1_{-0.014}^{+0.010}\right)$ & $18\left(1_{-0.5}^{+0.4}\right)$ \\
$h e p$ & $8.04(1 \pm 0.30)$ & $8.31(1 \pm 0.30)$ & $4.82\left(1_{-0.04}^{+0.05}\right)$ & $7.8(1 \pm 0.16)$ \\
${ }^{7} \mathrm{Be}$ & $5.00(1 \pm 0.07)$ & $4.56(1 \pm 0.07)$ & $5.00(1 \pm 0.03)$ & $4.86(1 \pm 0.12)$ \\
${ }^{8} \mathrm{~B}$ & $5.58(1 \pm 0.13)$ & $4.59(1 \pm 0.13)$ & $\leq 6.7$ & $5.79(1 \pm 0.23)$ \\
${ }^{13} \mathrm{~N}$ & $2.96(1 \pm 0.15)$ & $2.17(1 \pm 0.13)$ & $\leq 3.2$ & $5.71(1 \pm 0.36)$ \\
${ }^{15} \mathrm{O}$ & $2.23(1 \pm 0.16)$ & $1.56(1 \pm 0.15)$ & $\leq 5.9$ & $5.03(1 \pm 0.41)$ \\
${ }^{17} \mathrm{~F}$ & $5.52(1 \pm 0.18)$ & $3.40(1 \pm 0.16)$ & - & $5.91(1 \pm 0.44)$ \\
\hline$\chi^{2} / P^{\text {agr }}$ & $3.5 / 90 \%$ & $3.4 / 90 \%$ & & - \\
\hline \multicolumn{7}{c}{} & & &
\end{tabular}

on the production of solar neutrinos is out of the scope of the present paper, but can be found elsewhere [43].

SSM calculations of neutrino fluxes have been affected by developments in the input physics discussed in previous sections. The two areas that have the strongest impact on the neutrino fluxes predicted by models are changes in nuclear cross-sections and the new solar composition. In Table 4, we list the results for neutrino fluxes for the up-to-date SSMs SFII-GS98 and SFII-AGSS09. For comparison, we include, in the last column, results from the BP04 SSM [76].

The most striking difference is the large reduction in the ${ }^{13} \mathrm{~N}$ and ${ }^{15} \mathrm{O}$ fluxes between the SFII-GS98 and BP04 models, which use the same solar composition. This reduction comes as a result of the new determination of $S_{1,14}$, mostly by the LUNA experiment $[77,78]$, that has halved its value with respect to previous results (Table 2). If correct, the new expectation value of the combined ${ }^{13} \mathrm{~N}+{ }^{15} \mathrm{O}$ fluxes poses an even more challenging task for neutrino experiments to detect CNO fluxes. By comparing fluxes in Table 4 for models computed with the same solar composition (SFII-GS98 and BP04), it can be seen that in terms of flux values, those associated with the pp-chains have not changed much since 2004, despite improvements in the input physics entering solar model calculations. Few percent changes are present and are the result of changes in the nuclear cross-sections discussed before and also of the new OP radiative opacities. This is an encouraging situation; it implies that neutrino fluxes are robust predictions of solar models and, as experimental data on solar neutrinos accumulate, it will be possible to start fulfilling the initial goal posed by Davis and Bahcall: to use solar neutrinos to learn about the solar interior.

In Figure 2, we show the distribution of the solar neutrino fluxes as a function of solar radius. Together with the electron density profile, provided also by solar models (and neutron density profiles for sterile neutrino studies), these quantities are of fundamental importance for neutrino oscillation studies. It is worth noting that the ${ }^{13} \mathrm{~N}$ flux has two components. The larger one is associated with the operation in quasi-steady state of the $\mathrm{CN}$-cycle in the innermost solar core $\left(R<0.1 R_{\odot}\right)$, and for this reason coincides with the production region of the ${ }^{15} \mathrm{O}$ flux (Figure 2(b), blue and black curves, resp.). This component of the ${ }^{13} \mathrm{~N}$ flux, as well as the total ${ }^{15} \mathrm{O}$ flux, is linearly dependent on $\mathrm{S}_{1,14}$. The additional component of the ${ }^{13} \mathrm{~N}$ flux comes from the residual burning of ${ }^{12} \mathrm{C}$ by the reactions ${ }^{12} \mathrm{C}(p, \gamma){ }^{13} \mathrm{~N}\left(\beta^{+}\right){ }^{13} \mathrm{C}$ at temperatures not high enough to close the $\mathrm{CN}$-cycle with a proton capture on ${ }^{14} \mathrm{~N}$. This component is completely independent of $\mathrm{S}_{1,14}$. The careful reader will notice that the ratio of ${ }^{13} \mathrm{~N}$ and ${ }^{15} \mathrm{O}$ fluxes is different in the SFII-GS98 and BP04 models, despite having the same solar composition. Whereas the added ${ }^{13} \mathrm{~N}+{ }^{15} \mathrm{O}$ neutrino flux is linearly proportional to the $\mathrm{C}+\mathrm{N}$ abundance in the solar core and also linearly proportional to $\mathrm{S}_{1,14}$, this degeneracy can be broken, at least theoretically, if the two fluxes can be experimentally isolated from one another.

The impact of the low-Z solar composition on the production of solar neutrinos can be grasped by comparing results of models SFII-GS98 and SFII-AGSS09 shown in Table 4. As stated before, metals shape the solar structure through the radiative opacity. The lower abundance of metals in the AGSS09 composition is responsible for a reduction of the temperature in the solar core of about $1 \%$. Because of the extreme temperature sensitivity of some of the neutrino fluxes this is enough to produce large changes in the total fluxes. The most extreme case is, of course, ${ }^{8} \mathrm{~B}$, with the SFIIAGSS09 value being $\sim 20 \%$ smaller. For ${ }^{7} \mathrm{Be}$ the reduction is of $\sim 9 \%$.

Given the small uncertainties in the experimental determination of these fluxes, it would be tempting to think these neutrino fluxes have the potential to discriminate between the two flavors of solar composition and contribute, in this way, to the solution of the solar abundance problem. As can be seen in Table 4, unfortunately, the ${ }^{7} \mathrm{Be}$ and ${ }^{8} \mathrm{~B}$ fluxes determined from experiments lie almost right in between the high- $Z$ and low-Z models.

In any case, since it is known that low- $Z$ solar models do not reproduce well the solar structure as discussed in the previous section, it is dangerous to extract conclusions from comparing neutrino fluxes of this model to experimental results. Regardless of what the solution to the solar abundance problem is, since it will modify the solar interior structure, it will also change the expected values for the neutrino fluxes. In this regard, CNO fluxes are particularly interesting. Although 


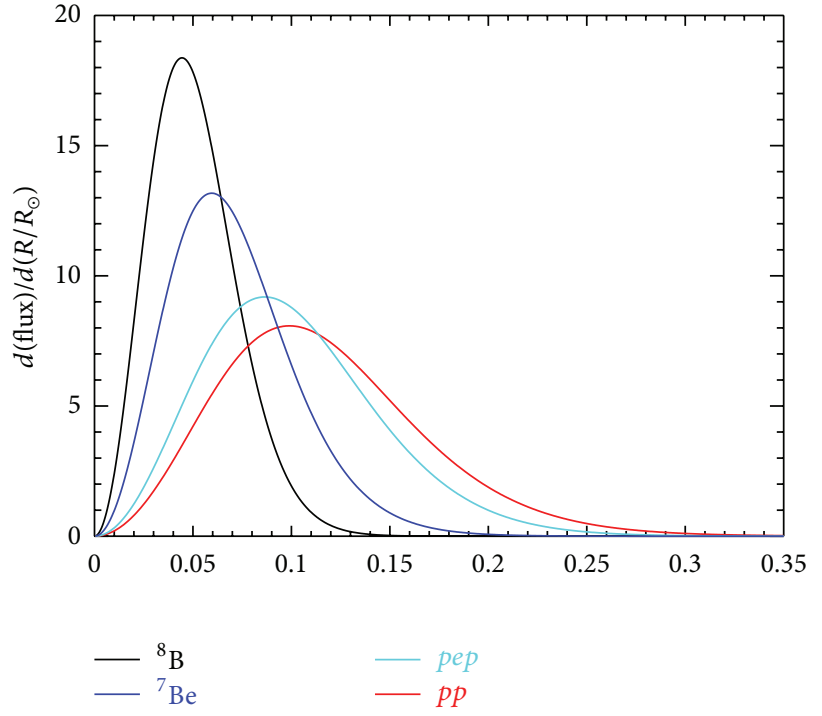

(a)

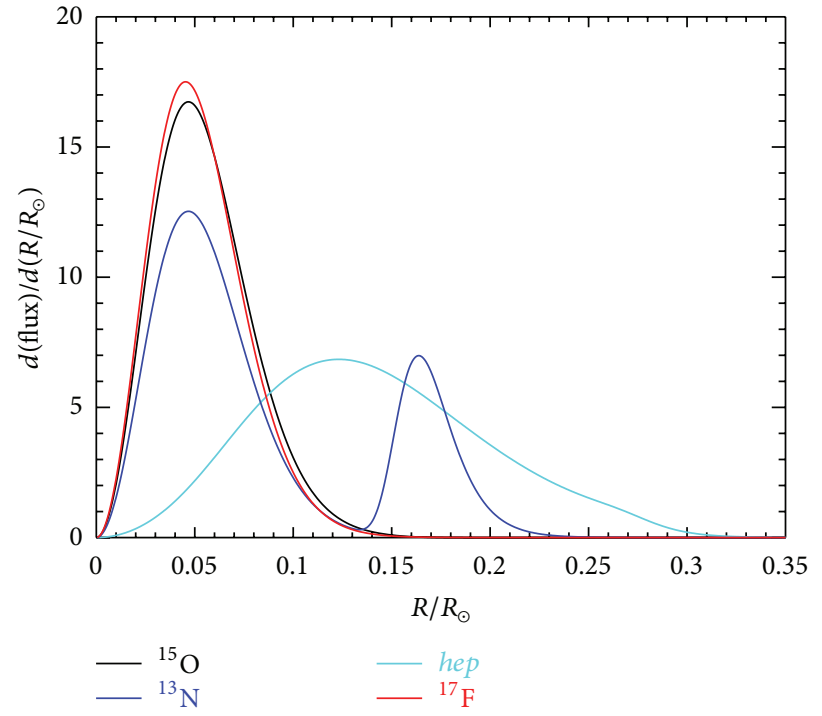

(b)

FIGURE 2: Normalized production profiles of solar neutrinos as a function of solar radius.

they are of course affected by temperature variations to a comparable degree as the ${ }^{8} \mathrm{~B}$ flux is, they carry an extra linear dependence on the solar composition that is not related to temperature variations (The ${ }^{17} \mathrm{~F}$ flux is linearly dependent on $\mathrm{O}$, but unfortunately the flux is too low to be detectable with current experimental capabilities.). Of particular interest is the linear dependence of the ${ }^{13} \mathrm{~N}$ and ${ }^{15} \mathrm{O}$ fluxes on the combined $\mathrm{C}+\mathrm{N}$ abundance. It is this dependence that enhances their capability as a diagnostic tool. In fact, differences between SFII-GS98 and SFII-AGSS09 models for these two fluxes are of the order of 30\% (taking SFII-GS98 as reference) and, what is more important, a large contribution to these differences does not have an origin on temperature differences between the models.

The last row in Table 4 shows the results of an $\chi^{2}$ test for the two models against the solar fluxes also shown in the table. It is clear that both SSMs give a very good agreement with current data. We emphasize again, however, that the four fluxes that are currently well determined from data and the luminosity constraint, depending on the solar composition only in an indirect manner. Experimental determination of the combined ${ }^{13} \mathrm{~N}+{ }^{15} \mathrm{O}$ flux will therefore provide qualitatively new information on the solar structure and composition. In fact, one can take advantage of the similar response to temperature variations that $\mathrm{CNO}$ fluxes and the ${ }^{8} \mathrm{~B}$ flux has. This has been exploited [79] to develop a very simple method to determine the solar core $\mathrm{C}+\mathrm{N}$ abundance that minimizes environmental uncertainties in solar models (i.e., sources of uncertainty that affect the solar core temperature). The idea is simple: the temperature dependences are cancelled out by using an appropriate ratio between the ${ }^{8} \mathrm{~B}$ and the combined ${ }^{13} \mathrm{~N}+{ }^{15} \mathrm{O}$ fluxes where SSM fluxes only act as normalization values and the overall scale is determined by an actual ${ }^{8} \mathrm{~B}$ flux measurement. The only additional requirement is that a measurement of the combined ${ }^{13} \mathrm{~N}+{ }^{15} \mathrm{O}$ flux becomes available. The current upper limit on this combined flux from Borexino [74] places an upper limit on the $\mathrm{C}+\mathrm{N}$ central mass fraction of $X_{\mathrm{C}+\mathrm{N}}<0.072$. Results for the SFII-GS98 and SFIIAGSS09 models are $X_{\mathrm{C}+\mathrm{N}}=0.048$ and 0.039 , respectively.

3.3.2. Uncertainties. Uncertainties in the model predictions of solar neutrino fluxes are given in Table 4. For deriving the total uncertainty basically two approaches can be used. On one hand, all contributions of uncertainty can be treated simultaneously by doing a Monte Carlo simulation [80]. The advantage is that intrinsic nonlinearities are captured in the total error. The disadvantage is that individual contributions to the total uncertainty are hardwired in the final result and cannot be disentangled. Fortunately, for the current level of uncertainties entering SSM calculations, nonlinearities seem to be negligible and the total uncertainty in neutrino fluxes can be obtained (adding quadratically) from individual contributions. To compute the latter, the expansion of fluxes as a product of power laws in the input parameters [43] around central values is a widely used, practical, insightful, and accurate approach. Uncertainties in the model fluxes listed in Table 4 have been obtained in this way.

The most important change introduced in the estimation of uncertainties is related to the treatment of the solar composition. Up until the BP04 model [76], the uncertainty in the solar composition was taken into account by considering variations of the total solar metallicity (to be more precise, changes in the $(Z / X)_{\odot}$ value used to construct SSMs). This leads to an overestimation of the neutrino uncertainties. The reason is that metals dominating the error budget in $(Z / X)_{\odot}$ $(\mathrm{C}, \mathrm{N}, \mathrm{O}$, and $\mathrm{Ne}$ ) have, at most, a moderate impact on the neutrino fluxes because of their small contribution to the radiative opacity, and therefore a rather small impact on temperature, in the region where most neutrinos are produced. On the other hand, elements such as Fe, S, and Si are 
second-order in determining $(Z / X)_{\odot}$ but play a fundamental role as sources of opacity in the solar core. It is important, therefore, to treat metal uncertainties individually [81]. Of course, in the case of the CNO fluxes the situation is different because CNO elements catalyze the CNO-bicycle and this overimposes an almost linear dependence of the ${ }^{13} \mathrm{~N}$ and ${ }^{15} \mathrm{O}$ on the $\mathrm{C}+\mathrm{N}$ content of the solar and a similar dependence of ${ }^{17} \mathrm{~F}$ on the $\mathrm{O}$ abundance. The uncertainties in the neutrino fluxes given for the SFII-GS98 and SFII-AGSS09 SSMs have been computed using the uncertainties for each relevant element given in the original publications $[46,50]$. As a result, for either family of solar models, that is, high- $Z$ or low- $Z$ models, the solar composition is not the dominant source of uncertainty for any of the fluxes of the pp-chains. In the case of the CNO fluxes, the linear dependence mentioned above is the dominant source of uncertainty: the combined $\mathrm{C}+\mathrm{N}$ abundance contributes to a $12 \%$ uncertainty for both the ${ }^{13} \mathrm{~N}$ and the ${ }^{15} \mathrm{O}$ fluxes, and the $\mathrm{O}$ abundance to $15 \%$ in the ${ }^{17} \mathrm{~F}$ flux.

In the case of the noncomposition uncertainties, the situation has improved in some cases thanks to more precise measurements of nuclear reaction rates. This is the case, in particular, for the ${ }^{3} \mathrm{He}\left({ }^{4} \mathrm{He}, \gamma\right)^{7} \mathrm{Be}$ reaction, which now contributes only $4.7 \%$ and $4.5 \%$ of the total uncertainty in the ${ }^{7} \mathrm{Be}$ and ${ }^{8} \mathrm{~B}$ fluxes, respectively. For comparison, the analogous contributions in the BP04 model were $8.0 \%$ and $7.5 \%$ [76]. Significant progress has also been achieved regarding ${ }^{14} \mathrm{~N}(p, \gamma){ }^{15} \mathrm{O}$, which now introduces uncertainties of only $5 \%$ and $7 \%$ in the ${ }^{13} \mathrm{~N}$ and ${ }^{15} \mathrm{O}$ fluxes, half the amount it did in 2004. An important contribution to the uncertainty in the ${ }^{8} \mathrm{~B}$ flux now comes from ${ }^{7} \mathrm{Be}(p, \gamma)^{8} \mathrm{~B}$ because the uncertainty of this reaction has been revised upwards [55]. Even if the uncertainty in this rate is now smaller than in SFI (see Table 2), it is larger than that used for the BP04 model, which was taken considering only one experimental result for this reaction.

While progress has been done in some cases, others have not seen much development, particularly diffusion and the delicate issue of radiative opacities. In Table 5, we give the individual contributions to flux uncertainties for the most relevant sources. The reader can compare directly to the situation in 2004 [76].

\section{Neutrino Flavor Conversion in Vacuum and Matter}

Neutrino flavor conversion has been reviewed by Yu. Smirnov in this volume and we refer the reader for a detailed physics discussion and references to his article. Here we just summarize the basic features and formulae of flavor conversion relevant to solar neutrinos.

We consider mixing of the three flavor neutrinos. The description of flavor conversion of solar neutrinos traveling through a medium is simplified because (a) the hierarchy in mass splittings determined by solar and atmospheric data leads to a reduction of the three neutrino flavor conversion to an effective two-flavor problem and (b) the neutrino parameters, the mixings, and solar mass splitting lead to adiabatic flavor conversion in solar matter and to cancel the interference term by averaging out. Therefore, the physics of the flavor conversion of solar neutrinos is described by simple expressions with a very good accuracy. In practice, the survival probability is computed numerically to correctly include the number density of scatterers along the trajectory of neutrinos from the production to the detection and to average over the neutrino production region. In solar neutrino flavor conversion, $v_{\mu}$ and $v_{\tau}$ are indistinguishable and therefore the survival probability of electron neutrinos is the only function needed to describe the flavor composition of the solar neutrino flux.

Solar neutrino survival or appearance probabilities can be expressed in terms of three oscillation parameters (that can be called the solar oscillation parameters), namely, the mixing angles between the first neutrino mass eigenstate and the two other active mass eigenstates $\left(\theta_{12}\right.$ and $\left.\theta_{13}\right)$ and the difference of the squares of the first and second generation mass eigenvalues $\left(\Delta m_{21}^{2}\right)$. The survival probability in the absence of Earth-matter effects, that is, during the day, is well described by

$$
P_{e e}^{D}=\cos ^{4} \theta_{13}\left(\frac{1}{2}+\frac{1}{2} \cdot \cos 2 \theta_{S} \cdot \cos 2 \theta_{12}\right)+\sin ^{4} \theta_{13} .
$$

Here, $\theta_{S}$ is the mixing angle at the production point inside the Sun:

$$
\cos 2 \theta_{S} \equiv \cos 2 \theta_{m}\left(\rho_{S}\right),
$$

where $\theta_{m}(\rho)$ is the mixing angle in matter of density $\rho_{S}$,

$$
\cos 2 \theta_{S}=\frac{\cos 2 \theta_{12}-\xi_{S}}{\left(1-2 \xi_{S} \cos 2 \theta_{12}+\xi_{S}^{2}\right)^{1 / 2}} .
$$

In (7), $\xi_{S}$ is defined as the ratio of the neutrino oscillation length in vacuum, $l_{v}$, to the refraction length in matter, $l_{0}$ :

$$
\begin{aligned}
\xi_{S} \equiv \frac{l_{v}}{l_{0}} & =\frac{2 \sqrt{2} G_{F} \rho_{S} Y_{e} \cos ^{2} \theta_{13}}{m_{N}} \frac{E}{\Delta m^{2}} \\
& =0.203 \times \cos ^{2} \theta_{13}\left(\frac{E}{1 \mathrm{MeV}}\right)\left(\frac{\rho_{S} Y_{e}}{100 \mathrm{~g} \mathrm{~cm}^{-3}}\right),
\end{aligned}
$$

where

$$
l_{v} \equiv \frac{4 \pi E}{\Delta m^{2}}, \quad l_{0} \equiv \frac{2 \pi m_{N}}{\sqrt{2} G_{F} \rho_{S} Y_{e} \cos ^{2} \theta_{13}} .
$$

In (8) and (9), $\rho_{S}$ is the solar matter density, $Y_{e S}$ is the number of electrons per nucleon, and $m_{N}$ is the nucleon mass. The electron solar density and neutrino production distribution of the neutrino fluxes are derived from solar models as discussed in the previous section. In the last line in (8), we have used the best fit values of the global analysis $\Delta m^{2}=7.5 \times$ $10^{-5} \mathrm{eV}^{2}$. The ratio of the parameter $\rho_{S}$ to $\cos 2 \theta_{12}$ separates the region where the flavor conversion corresponds to vacuum averaged oscillations from the one of matter dominated conversion. 
TABle 5: Percentage contribution of selected individual sources of uncertainty to the neutrino fluxes.

\begin{tabular}{|c|c|c|c|c|c|c|c|}
\hline & $\mathrm{S}_{11}$ & $\mathrm{~S}_{33}$ & $\mathrm{~S}_{34}$ & $\mathrm{~S}_{17}$ & $S_{1,14}$ & Opac & Diff \\
\hline$p p$ & 0.1 & 0.1 & 0.3 & 0.0 & 0.0 & 0.2 & 0.2 \\
\hline pep & 0.2 & 0.2 & 0.5 & 0.0 & 0.0 & 0.7 & 0.2 \\
\hline hep & 0.1 & 2.3 & 0.4 & 0.0 & 0.0 & 1.0 & 0.5 \\
\hline${ }^{7} \mathrm{Be}$ & 1.1 & 2.2 & 4.7 & 0.0 & 0.0 & 3.2 & 1.9 \\
\hline${ }^{8} \mathrm{~B}$ & 2.7 & 2.1 & 4.5 & 7.7 & 0.0 & 6.9 & 4.0 \\
\hline${ }^{13} \mathrm{~N}$ & 2.1 & 0.1 & 0.3 & 0.0 & 5.1 & 3.6 & 4.9 \\
\hline${ }^{15} \mathrm{O}$ & 2.9 & 0.1 & 0.2 & 0.0 & 7.2 & 5.2 & 5.7 \\
\hline${ }^{17} \mathrm{~F}$ & 3.1 & 0.1 & 0.2 & 0.0 & 0.0 & 5.8 & 6.0 \\
\hline
\end{tabular}

The $v_{e}$ survival probability at night during which solar neutrinos pass through the Earth can be written as

$$
P_{e e}^{N}=P_{e e}^{D}-\cos 2 \theta_{S} \cos ^{2} \theta_{13}\left\langle f_{\text {reg }}\right\rangle_{\text {zenith }}
$$

where $P_{e e}^{D}$ is the one given in (5). $f_{\text {reg }}$ denotes the regeneration effect in the Earth and is given as $f_{\text {reg }}=P_{2 e}-\sin ^{2} \theta_{12} \cos ^{2} \theta_{13}$, where $P_{2 e}$ is the transition probability of the second mass eigenstate to $v_{e}$. Under the constant density approximation in the Earth, $f_{\text {reg }}$ is given by

$$
\begin{aligned}
f_{\text {reg }}= & \xi_{E} \cos ^{2} \theta_{13} \sin ^{2} 2 \theta_{E} \\
& \times \sin ^{2}\left[a_{E} \cos ^{2} \theta_{13}\left(1-2 \xi_{E}^{-1} \cos ^{2} \theta_{12}+\xi_{E}^{-2}\right)^{1 / 2}\left(\frac{L}{2}\right)\right],
\end{aligned}
$$

for passage of distance $L$, where we have introduced $a_{E} \equiv$ $\sqrt{2} G_{F} n_{e}^{\text {Earth }}=\sqrt{2} G_{F} \rho_{E} Y_{e E} / m_{N}$.

In (11), $\theta_{E}$ and $\xi_{E}$ stand for the mixing angle and the $\xi$ parameter (see (8)) with matter density $\rho_{E}$ in the Earth. Within the range of neutrino parameters allowed by the solar neutrino data, the oscillatory term averages to $1 / 2$ in a good approximation when integrated over zenith angle. Then, the equation simplifies to

$$
\left\langle f_{\text {reg }}\right\rangle_{\text {zenith }}=\frac{1}{2} \cos ^{2} \theta_{13} \xi_{E} \sin ^{2} 2 \theta_{E} .
$$

At $E=7 \mathrm{MeV}$, which is a typical energy for ${ }^{8} \mathrm{~B}$ neutrinos, $\xi_{E}=$ $3.98 \times 10^{-2}$ and $\sin 2 \theta_{E}=0.940$ for the average density $\bar{\rho}_{E}=$ $5.6 \mathrm{~g} / \mathrm{cm}^{3}$ and the electron fraction $Y_{e E}=0.5$ in the Earth. Then, $\left\langle f_{\text {reg }}\right\rangle_{\text {zenith }}$ is given as $\left\langle f_{\text {reg }}\right\rangle_{\text {zenith }}=1.76 \times 10^{-2}$ for the best fit neutrino parameters. This result is in a reasonable agreement with the computed Earth-matter factor using the best estimates on the Earth-matter density.

\section{Recent Solar Neutrino Measurements}

5.1. The SNO and SK Legacy. After the results and analyses from 2002, it was clear that the LMA oscillation was the right solution of the long standing solar neutrino puzzle [82-93], but the activity of the SNO and SK experiments continued in the following years. The data obtained from these experiments were very important in making the LMA solution more robust and in improving the accuracy and precision of the mixing parameters determination.

The so-called SNO II experiment began in June of 2001 with the addition of $2000 \mathrm{~kg}$ of $\mathrm{NaCl}$ to the 1000 metric tons of $\mathrm{D}_{2} \mathrm{O}$ and ended in October 2003 when the $\mathrm{NaCl}$ was removed. The addition of salt significantly increased SNO's efficiency (by a factor $\sim 3$ with respect to the pure $\mathrm{D}_{2} \mathrm{O}$ phase) in the detection of neutrons produced in the neutral current (NC) disintegration of deuterons by solar neutrinos and, by enhancing the energy of the $\gamma$-ray coming from neutron capture, allowed a more precise measurement of this interaction channel, well above the low-energy radioactive background. Moreover, the isotropy of the multiple $\gamma$-ray emission by neutron capture on ${ }^{35} \mathrm{Cl}$ is different from the one of the Črenkov light emitted by the single electron of the charged current interaction; therefore, by studying the event isotropy, it has been possible to separate the neutral from the charged current events without any additional assumption on the neutrino energy spectrum. The salt phase results have been reported in two main publications. In [30], referring to the first 254 live days, a global analysis including all the solar and reactor neutrino results rejected the maximal mixing hypothesis at a $5.4 \sigma$ level and gave a value of the ${ }^{8} \mathrm{~B}$ neutrino flux in agreement with previous measurements and with SSMs. These results were essentially confirmed (even if with a small shift towards larger values of the mixing angle) by the second publication [94], which included the full data of the salt phase (391 live days), analyzed in terms of the CC spectra (starting from $5.5 \mathrm{MeV}$ kinetic energy), and $\mathrm{NC}$ and ES integrated fluxes separately for day and night. The day-night asymmetry in the neutral current rate, which would be an indication of oscillation to sterile neutrinos or nonstandard interaction with matter in the earth, came out to be consistent with zero.

This result confirmed also the outcome of the study performed for elastic scattering (ES) interaction above $5 \mathrm{MeV}$ by the Super-Kamiokande collaboration [95]. The full SK-I low energy data, corresponding to 1496 live days until July 2001, were investigated analyzing the time variations of the ES rates and fitting them to the variations expected from active two neutrino oscillations. In this kind of study, the zenith angle of the solar neutrinos arriving on the detector is associated to the time variable and the full data are divided in two different time samples from which the day and night rates (D) and $(\mathrm{N})$ are derived. The day-night asymmetry turned out to be $A_{\mathrm{DN}}=2(\mathrm{D}-\mathrm{N}) /(\mathrm{D}+\mathrm{N})=-0.021 \pm 0.020$ (stat. $)_{-0.012}^{+0.013}$ (syst.), 
which is consistent with zero within $0.9 \sigma$. This value was in a good agreement also with the LMA oscillation solution, which (for the best fit parameter) predicted [95] $A_{\mathrm{DN}}=$ $-0.018 \pm 0.016$ (stat.) ${ }_{-0.012}^{+0.013}$ (syst.). The impact of Earth-matter effects on solar neutrino oscillations is an important topic, which has been widely studied in the literature with different techniques (analytical and semianalytical studies and fully numerical analyses) [96-109].

The SK analysis $[95,110]$ also showed that the energy spectrum of the recoiling electron was consistent with an undistorted solar ${ }^{8} \mathrm{~B}$ neutrino spectrum and did not find any anomalous periodic time variation of the rates, apart from the expected seasonal variation due to the Earth's orbit eccentricity. The SK best fit point was in quite a good agreement with the SNO results, even if SK would favor slightly larger values of $\tan ^{2} \theta$. A SNO-only analysis gave the following best fit parameters [94]: $\Delta m_{12}^{2}=5.0 \times 10^{-5} \mathrm{eV}^{2}, \tan ^{2} \theta_{12}=0.45$. Including all the other solar neutrino and the KamLAND results, the best fit was obtained for $\Delta m_{12}^{2}=8.0_{-0.4}^{+0.6} \times 10^{-5} \mathrm{eV}^{2}$, $\tan ^{2} \theta_{12}=0.452_{-0.070}^{+0.088}$. The effect of KamLAND data was mainly to increase the value of $\Delta m^{2}$ and to restrict the allowed region in the mixing parameter plane. The main difference of the global analysis done with the SNO salt phase data with respect to previous studies was the possibility to exclude at 95\% CL the secondary region at even larger values of the mass differences (the so-called LMA II solution, with $\Delta m_{12}^{2}>$ $\left.10^{-4} \mathrm{eV}^{2}\right)$.

In the third SNO phase (November 2004-November 2006), the neutral current signal neutrons were mainly detected by means of an array of ${ }^{3} \mathrm{He}$ proportional counters deployed in the $\mathrm{D}_{2} \mathrm{O}$ and looking at the gas ionization induced by neutron capture on ${ }^{3} \mathrm{He}$. In this way, the fluxes correlation was reduced and the accuracy in the mixing angle determination was improved. The total active ${ }^{8} \mathrm{~B}$ neutrino flux was found [31] to be $5.54_{-0.31}^{+0.33}$ (stat. $)_{-0.34}^{+0.36}$ (syst.) $\times$ $10^{6} \mathrm{~cm}^{-2} \mathrm{~s}^{-1}$, in agreement with previous measurements and SSMs. The ratio of the ${ }^{8} \mathrm{~B}$ neutrino flux measured with $\mathrm{CC}$ and NC reaction was $\Phi_{\mathrm{CC}}^{\mathrm{SNO}} / \Phi_{\mathrm{NC}}^{\mathrm{SNO}}=0.301 \pm 0.033$. The global solar neutrino experiment analysis included, in this case, also the first results coming from the Borexino experiment [111], that we discuss in Section 5.3. The best fit point moved to $\Delta m_{12}^{2}=4.90 \times 10^{-5} \mathrm{eV}^{2}, \tan ^{2} \theta_{12}=0.437$ and the uncertainty in the mixing parameter plane was still quite large. Adding the KamLAND data, the allowed region was significantly restricted (mainly for $\Delta m^{2}$ ) and the marginalized $1 \sigma$ regions were $\Delta m_{12}^{2}=7.59_{-0.21}^{+0.19} \times 10^{-5} \mathrm{eV}^{2}, \tan ^{2} \theta_{12}=0.469_{-0.041}^{+0.047}$.

A subsequent joint reanalysis of SNO I and SNO II data, known as LETA (low energy threshold analysis) [112], succeeded, with improved calibration and analysis techniques, in lowering the energy threshold, with respect to previous analyses [94, 113], down to an effective electron kinetic energy of $T_{\text {eff }}=3.5 \mathrm{MeV}$. The main effect was to increase the statistics of CC and ES and, above all, of NC events and to increase significantly the precision on both the total ${ }^{8} \mathrm{~B}$ neutrino flux and the neutrino mixing parameters. The value for the total ${ }^{8} \mathrm{~B}$ neutrino flux extracted from neutral current was $\Phi_{\mathrm{NC}}=5.14_{-0.20}^{+0.21} \times 10^{6} \mathrm{~cm}^{-2} \mathrm{~s}^{-1}$, where the error, obtained by summing in quadrature the statistic and systematic contributions, was reduced by more than a factor of two with respect to previous publications. For SNO data alone (LETA plus SNO III), the best fit point moved to the LOW region of parameter space, but the significance level was very similar to the one of the usual LMA solution. A global fit, including all the solar and the KamLAND data, essentially confirmed, instead, the previous results [31] for $\Delta m_{12}^{2}$ and it made possible a further improvement in the angle determination, giving, in a 2-flavor analysis, $\tan ^{2} \theta_{12}=0.457_{-0.028}^{+0.041}$.

In the last five years, also the Super-Kamiokande collaboration presented new analyses, including the data of the different working phases of this experiment: Super-Kamiokande II [114] (from December 2002 to October 2005) and Super-Kamiokande III (from July 2006 to August 2008) [115]. Due to the 2001 accident, which damaged some of the photomultiplier tubes, the detector sensitivity was reduced with respect to SK-I and therefore it was important to improve the methods adopted for data collection (particularly for vertex event reconstruction, angular resolution, and background reduction) and analysis. In this way, during the 548 days of SK-III a $2.1 \%$ systematic uncertainty on the total flux (corresponding roughly to two-thirds of the SK-I value) was reached. The second and third Super-Kamiokande phases essentially confirmed the SK-I results, for what concerns the absence of significant spectral distortion, the total ${ }^{8} \mathrm{~B}$ measured flux and the day-night asymmetry.

Since September 2008, Super-Kamiokande is running with modernized data acquisition system (DAQ) and electronics, which allow a wider dynamic range in the measured charge and is read out via Ethernet. This phase of the experiment is denoted as Super-Kamiokande IV [116]. Thanks to the fast DAQ every hit can be recorded and the resulting data stream analyzed by an online computer system that finds timing coincidences which are saved as triggers. As a consequence, Super-Kamiokande's energy threshold is now only limited by computing speed and the event reconstruction. The present event reconstruction is able to reconstruct electrons with a total energy of $3 \mathrm{MeV}$ or more. The computing speed limits the energy threshold to $4.2 \mathrm{MeV}$ which is just below the threshold of Super-Kamiokande I and III $(4.5 \mathrm{MeV})$. The same water flow techniques developed during Super-Kamiokande III result in an observed solar neutrino elastic scattering peak between 4 and $4.5 \mathrm{MeV}$ total recoil electron energy. Special techniques are developed to discriminate the signal from the background, taking advantage from the fact that the background is mainly due to $\beta$ emission from ${ }^{214} \mathrm{Bi}$ and it is characterized by a larger Coulomb multiple scattering. This makes possible a reduction of about $10-15 \%$ of the statistical uncertainty and this method can also be applied to previous phases of the experiment. The additional systematic uncertainty of this method is under investigation.

\subsection{The Impact of KamLAND Results on Solar Neutrino} Physics. Even if it is based on the analysis of a reactor antineutrino beam, the KamLAND experiment played a fundamental role in the solution of the long standing solar 
neutrino puzzle. In fact, the first KamLAND data [34] were determinant, in conjunction with the previous solar neutrino experiments (and mainly with SNO) and assuming CPT invariance, to prove the validity of the oscillation hypothesis and to select the LMA solution as the correct one.

Between March 2002 and January 2004, a new set of data were collected and the KamLAND collaboration performed a study including also a reanalysis of the previous data. During the 2002-2004 campaign, important upgrades were done both on the central detector (increasing the photocatode coverage and improving the energy resolution) and in the analysis techniques (reduction of the background with better techniques in the event selection cuts based on the time, position, and geometry of the events). The number of antineutrino events above $2.6 \mathrm{MeV}$ expected in absence of antineutrino disappearance was $365.2 \pm 23.7$ (syst) and the 258 observed events corresponded to a $\bar{v}_{e}$ survival probability equal to $0.658 \pm 0.044$ (stat.) \pm 0.047 (syst.). The energy spectrum analysis was in disagreement with the no oscillation hypothesis at $99.6 \%$ statistical significance. In [39], the KamLAND collaboration, looking at the $L_{0} / E$ spectrum dependence (where $L_{0}$ is the source-detector distance and $E$ the $\bar{v}_{e}$ energy), performed also an interesting study of other alternative hypotheses (like decoherence and decay) for neutrino disappearance. The oscillation hypothesis offered by far the best explanation of the spectrum shape, as one can see from Figure 3.

As shown in Figure 4(a), the best fit obtained from the data analysis was in the so-called LMAI region (with values of $\Delta m_{12}^{2}$ around $8 \cdot 10^{-5} \mathrm{eV}^{2}$ ) and the alternative solution at higher $\Delta m_{12}^{2}$ (around $2 \cdot 10^{-4} \mathrm{eV}^{2}$ ) was strongly disfavoured, at $98 \% \mathrm{CL}$, mainly due to the spectrum distortions. The KamLAND data alone were not sufficient to solve completely the ambiguity on the mixing angle values and to exclude maximal mixing. However, including in the analysis also the results coming from solar neutrino experiments, the allowed values of the angle were significantly restricted (see Figure 4(b)) and the two-flavor combined analysis gave $\Delta m_{12}^{2}=7.9_{-0.5}^{+0.6} \cdot 10^{-5} \mathrm{eV}^{2}, \tan ^{2} \theta_{12}=0.40_{-0.07}^{+0.10}$ at a $1 \sigma$ level.

The next KamLAND analysis [40] included also, in addition to the one of $[34,39]$, the new data collected up to May 2007. The increase in data collection was significant (also thanks to enlarging the radius of the fiducial volume from 5.5 to $6 \mathrm{~m}$ ) and there was a reduction of systematic uncertainties, in the number of target protons and the background. The total uncertainty on $\Delta m_{21}^{2}$ was around $2 \%$, mainly due to the distortion of the energy scale in the detector. The total uncertainty, $4.1 \%$, on the expected event rate was due to different sources (above all the definition of the detector fiducial volume and energy threshold, the $\bar{v}_{e}$ spectra, and the reactor power) and it affected primarily the mixing angle determination. The different background sources were studied and reduced further. The most important one was the ${ }^{13} \mathrm{C}(\alpha, n){ }^{16} \mathrm{O}$ reaction made possible by the $\alpha$ decay of ${ }^{210} \mathrm{Po}$ (a daughter of ${ }^{222} \mathrm{Rn}$ ) introduced in the liquid scintillator during the construction, which produces neutrons with energies up to 7.3 MeV.

The results of the statistical analysis are reported in Figure 5, taken from [40]. The allowed oscillation parameter

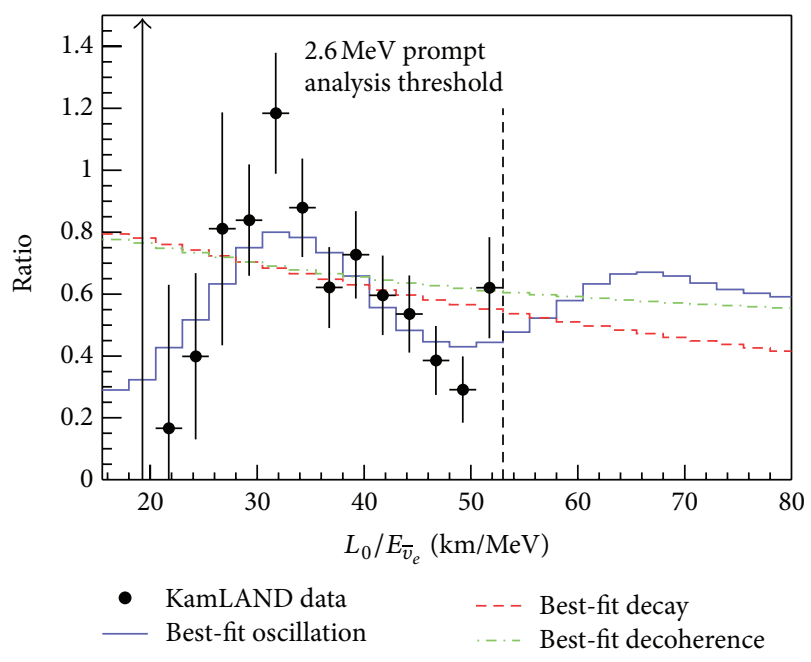

Figure 3: Ratio of the observed $\bar{v}_{e}$ spectrum to the expectation for nooscillation versus $L_{0} / E$. The curves show the expectation for the best-fit oscillation, best-fit decay, and best-fit decoherence models, taking into account the individual time-dependent flux variations of all reactors and detector effects. Taken from [39].

values were $\Delta m_{21}^{2}=7.58_{-0.13}^{+0.14}$ (stat. $)_{-0.15}^{+0.15}$ (syst.) $\cdot 10^{-5} \mathrm{eV}^{2}$ for the mass eigenvalues and $\tan ^{2} \theta_{12}=0.56_{-0.07}^{+0.10}$ (stat. $)_{-0.06}^{+0.10}$ (syst.), for $\tan ^{2} \theta_{12}<1$ and the no oscillation hypothesis was excluded at $5 \sigma$. The extension to the three-neutrino oscillation analysis had the main effect to enlarge the uncertainty on $\theta_{12}$, leaving $\Delta m_{12}^{2}$ substantially unchanged. Figure 5 , taken from [40], shows that the effect of the inclusion in the analysis of the data from SNO [94] and previous solar neutrino experiments was essential to reduce the interval of allowed $\theta_{12}$ values and also to move the best-fit point towards slightly lower values of the mixing angle.

Figure 6 (taken from [40]) illustrates, instead, the $\bar{v}_{e}$ survival probability, as a function of the ratio $L_{0} / E$ between the average baseline and the antineutrino energies. One can notice that the observed spectrum (after subtraction of background and geoneutrino signals), reproduces correctly the general shape of the expected oscillation cycle, with a slight excess of low energy antineutrinos, that could be interpreted as geoneutrinos.

\subsection{Toward the Sub-MeV Analysis: The Borexino Detector} and Its Measurements. In the last decade, significant steps forward have been done in the knowledge of solar neutrino properties, thanks mainly to the results obtained by the kiloton scale Čerenkov detectors (SK and SNO) and by advent of the reactor neutrino experiment KamLAND. However, these experiments investigated only the energy part of solar neutrino spectrum above $5 \mathrm{MeV}$, which represents a small fraction of the full spectrum. The single components of the neutrino spectrum cannot be determined by such techniques at low energies and, therefore, up to the last four years, low energy neutrinos had been observed only via radiochemical methods. A significant change took place with the advent of Borexino, a real-time experiment which opened the way to 


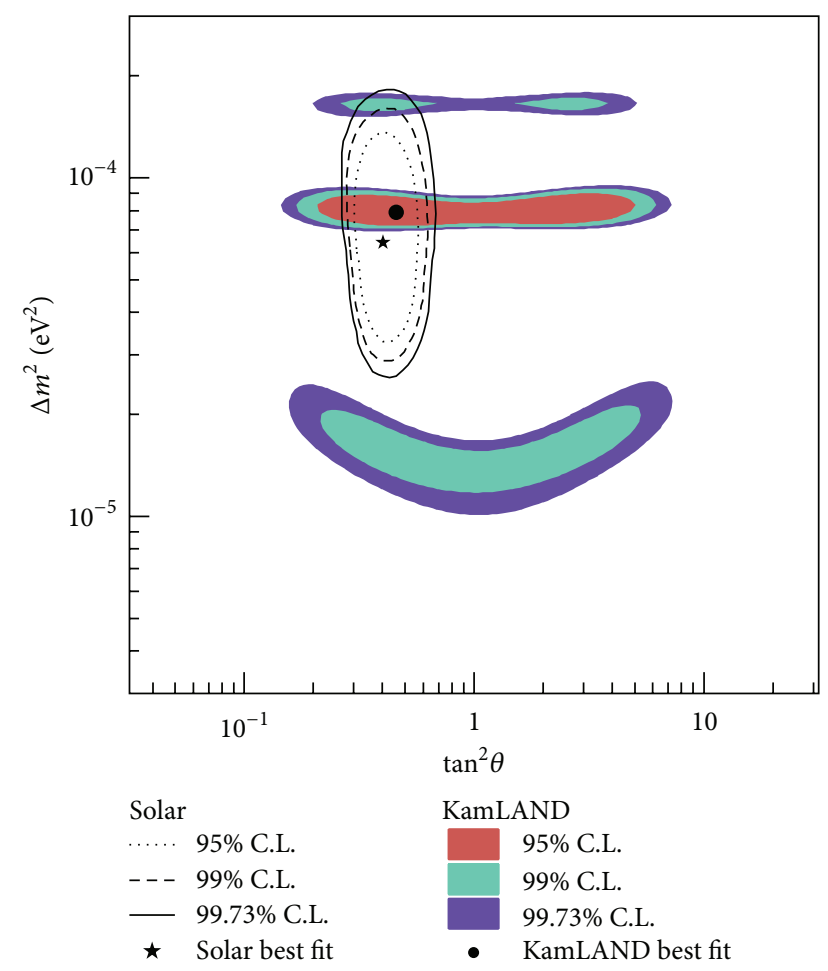

(a)

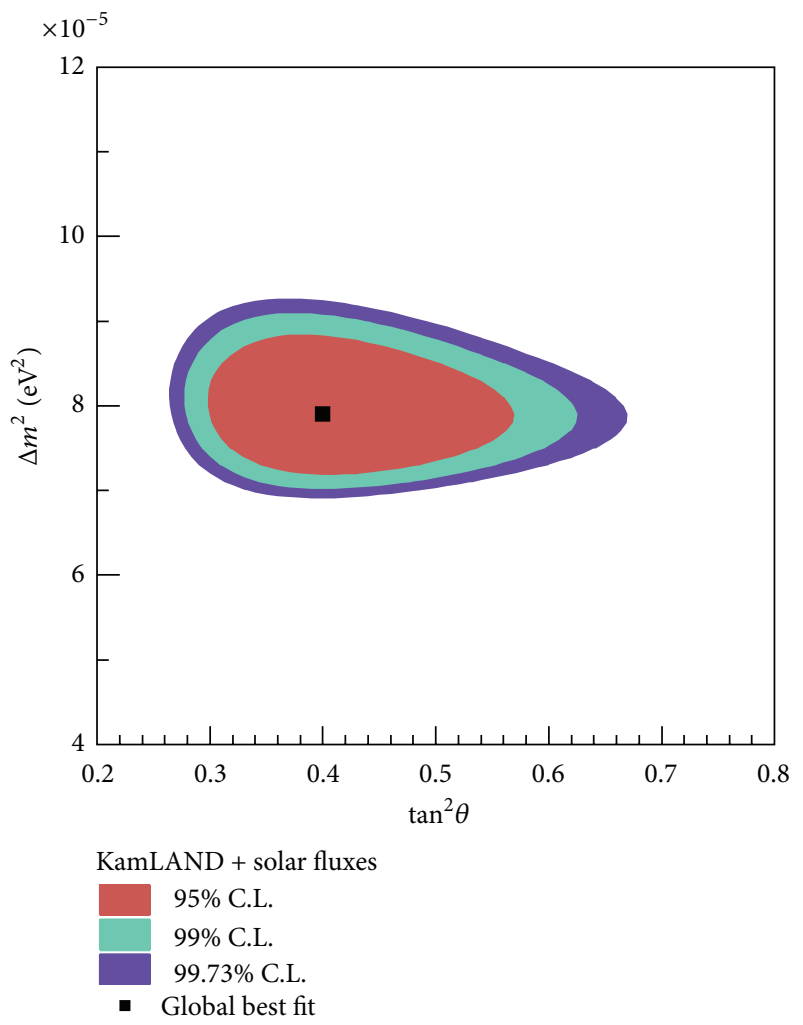

(b)

FIGURE 4: (a) Allowed region of the neutrino oscillation parameter from KamLAND antineutrino data (colored regions) and solar neutrino experiments (lines) [30]. (b) the result of a combined twoneutrino oscillation analysis of KamLAND and the observed solar neutrino fluxes under the assumption of CPT invariance. Taken from [39]. the investigation of the sub- $\mathrm{MeV}$ region and isolated for the first time the neutrinos corresponding to the monochromatic beryllium line.

5.3.1. The Borexino Detector. Borexino is an ultrahigh radiopure large volume liquid scintillator detector (using pseudocumene-PC (1,2,4-trimethylbenzene)-as aromatic scintillation solvent, and PPO (2,5-diphenyloxazole) as solute at a concentration of $1.5 \mathrm{~g} / \mathrm{L}$ ) located underground at the italian Gran Sasso National Laboratories (LNGS), under about $1400 \mathrm{~m}$ of rock (3800 mwe) [117]. The employment of a liquid scintillator as target mass assures a light production sufficient to observe low energy neutrino events via elastic scattering by electrons. This reaction is sensitive to all neutrino flavors, through the neutral current interaction, but the cross-section for $v_{e}$ is larger than $v_{\mu}$ and $v_{\tau}$ by a factor of 5-6, due to the combination of charged and neutral currents. The main goal of Borexino is the measurement of the monoenergetic $(0.862 \mathrm{MeV})^{7} \mathrm{Be}$ neutrinos, which have the basic signature of the Compton-like edge of the recoil electrons at $665 \mathrm{keV}$ (see Figure 7).

The high light yield typical of a liquid scintillator makes it possible to reach a low energy threshold, a good energy resolution of about $5 \%$ at $1 \mathrm{MeV}$, and a pulse shape discrimination between $\alpha$ and $\beta$ decays. On the other hand, no directionality is possible and it is also not possible to distinguish neutrino scattered electrons from electrons due to natural radioactivity. For this reason, an extremely low level of radioactive contamination is compulsory and this has been one of the main tasks and technological achievements of the experiment. The background due to the presence of $\beta$ decay of ${ }^{14} \mathrm{C}\left(\beta_{\text {end-point }} 156 \mathrm{keV}\right)$, intrinsic to the scintillator, limits neutrino observation to energies above $200 \mathrm{keV}$. Techniques for the scintillator purification are based mainly on methods developed and tested in earlier studies with the counting test facility (CTF), a 4-ton prototype of Borexino which demonstrated for the first time the feasibility of achieving the low backgrounds needed to detect solar neutrinos in a largescale scintillator [120-122]. For Borexino, a larger purification plant was developed similar to the CTF system, but with several improved features including the use of high vacuum and precision cleaning techniques.

The design of Borexino is based on the principle of graded shielding (onion-like structure-see Figure 8).

The scintillator ( $\approx 300$ tons) is contained in a thin nylon inner vessel (IV), of radius $4.25 \mathrm{~m}$, at the center of a set of concentric shells of increasing radiopurity and it is surrounded by an outer vessel (OV), filled with $\mathrm{PC}$ and $5.0 \mathrm{~g} / \mathrm{L}$ DMP (dimethyl phthalate), a material which is able to quench the residual scintillation of PC and acts as a passive shield against radon and other background contaminations originating from the external parts. A third more external vessel is composed of a stainless steel sphere (SSS), enclosing the passive shield (PC-DMP), and the entire detector is contained in a dome-shape structure $16.9 \mathrm{~m}$ high with a radius of $9 \mathrm{~m}$, filled with ultrapure water, denominated water tank (WT). The scintillation light is recorded by 22128 -inches photomultipliers distributed on the inner part of the SSS [123, 124]; 


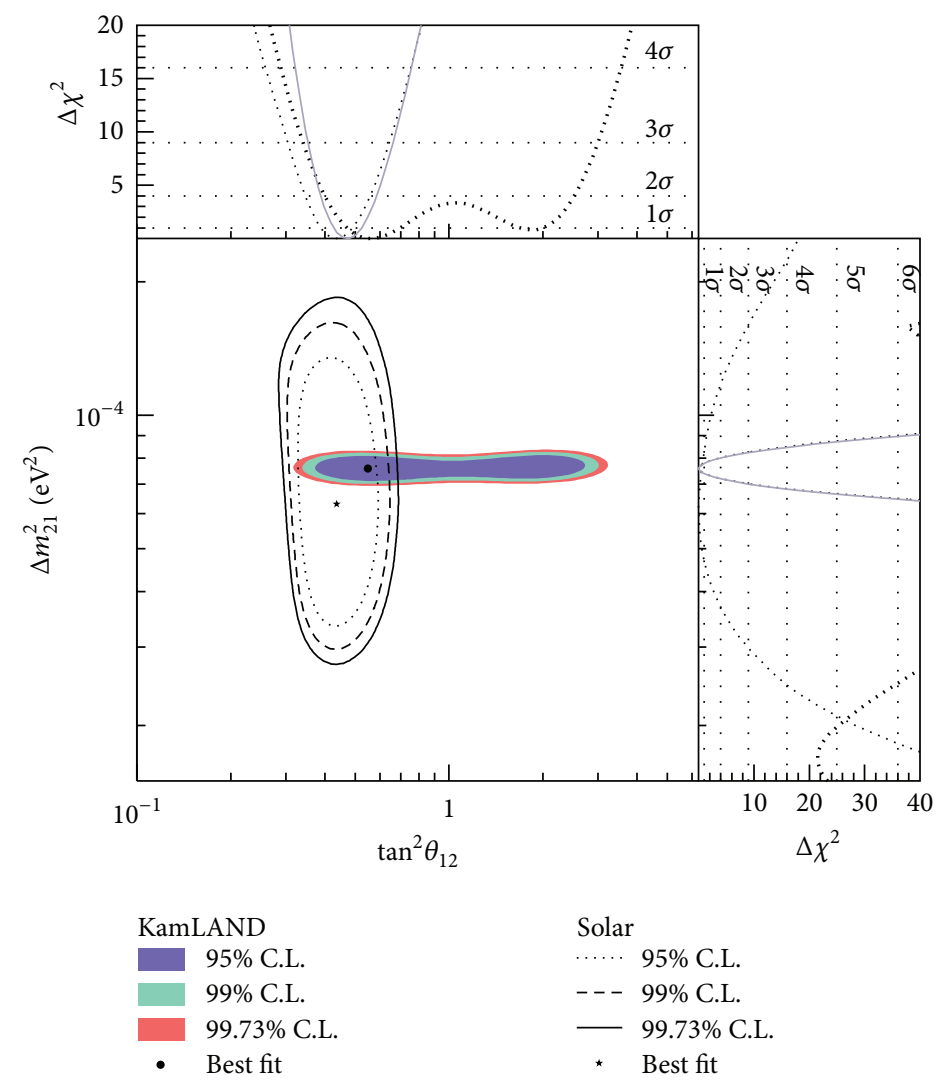

FiguRE 5: Allowed region for neutrino oscillation parameters from KamLAND and solar neutrino experiments. The side-panels show the $\Delta \chi^{2}$-profiles for KamLAND (dashed) and solar experiments (dotted) individually, as well as the combination of the two (solid). Taken from [40].

1828 of them are equipped with aluminum light concentrators designed to increase the light collection efficiency [125]. Čerenkov's light and residual background scintillation in the buffer are thus reduced. The others 384 photomultipliers without concentrators are used to study this background and to identify muons that cross the buffer and not the inner vessel. The water tank is equipped with 208 8-inches photomultipliers and acts as a Čerenkov muon detector. Although the muon flux is reduced by a six-order of magnitude by the 3800 mwe depth of the Gran Sasso Laboratory is still significant $\left(1.1 \mu \mathrm{m}^{-2} \mathrm{~h}^{-1}\right)$. An additional reduction, of the order of about $10^{4}$, has been necessary; for more details see [126].

In order to remove contaminants from dust (U, Th, K), air $\left({ }^{39} \mathrm{Ar},{ }^{85} \mathrm{Kr}\right)$, and cosmogenically produced isotopes $\left({ }^{7} \mathrm{Be}\right)$, different purification techniques were applied, such as distillation, water extraction, nitrogen stripping, and ultrafine filtration. The pseudocumene was distilled in-line during the detector filling at $80 \mathrm{mbar}$ and at a temperature of about $90-$ $95^{\circ} \mathrm{C}$. Distilled pseudocumene was stripped in an $8 \mathrm{~m}$ high (15 cm in diameter) packed column with specially prepared ultralow Ar/Kr nitrogen (0.005 ppm Ar and 0.06 ppt Kr, see [127]). Position reconstruction of the events, as obtained from the photomultipliers timing data via a time-of-flight algorithm, allowed to define a fiducial spherical volume, corresponding approximately to $1 / 3$ (i.e., about 100 tons) of the scintillator volume in order to reject external $\gamma$ background. The others $2 / 3$ of the scintillator act as an active shield.

5.3.2. The Measurement of the ${ }^{7}$ Be Line. The Borexino collaboration started taking data in May 2007 and after only 3 months (47.4 live days) it was able to extract the ${ }^{7} \mathrm{Be}$ signal from the background. The best value estimate for the rate was $47 \pm 7$ (stat.) \pm 12 (syst.) counts/(day 100 ton), where the systematic error is mainly due to the fiducial mass determination [119]. An update of the ${ }^{7} \mathrm{Be}$ signal was reported after 9 months from an analysis of 192 live days (from May 16 2007 to April 12 2008), corresponding to 41.3 ton $\cdot$ yr fiducial exposure to solar neutrinos.

The severe cuts that had to be passed by the events in order to be selected and enter the analysis were mainly designed to avoid pile up of multiple events and reject the events originated by muons and their daughters and the ones due to radon daughters preceding the $\alpha-\beta$ Bi-Po delayed coincidences. Moreover, severe cuts (radial and based on the $z$-coordinates) were finalized to reduce the external $\gamma$ background. The remaining fiducial mass was of about 79 metric tons. Important background sources were the fast coincidence decays from the ${ }^{238} \mathrm{U}$ chain (contamination level of $(1.6 \pm 0.1) 10^{-17} \mathrm{~g} / \mathrm{g}$ ) and the ${ }^{232} \mathrm{Th}$ chain (contamination 


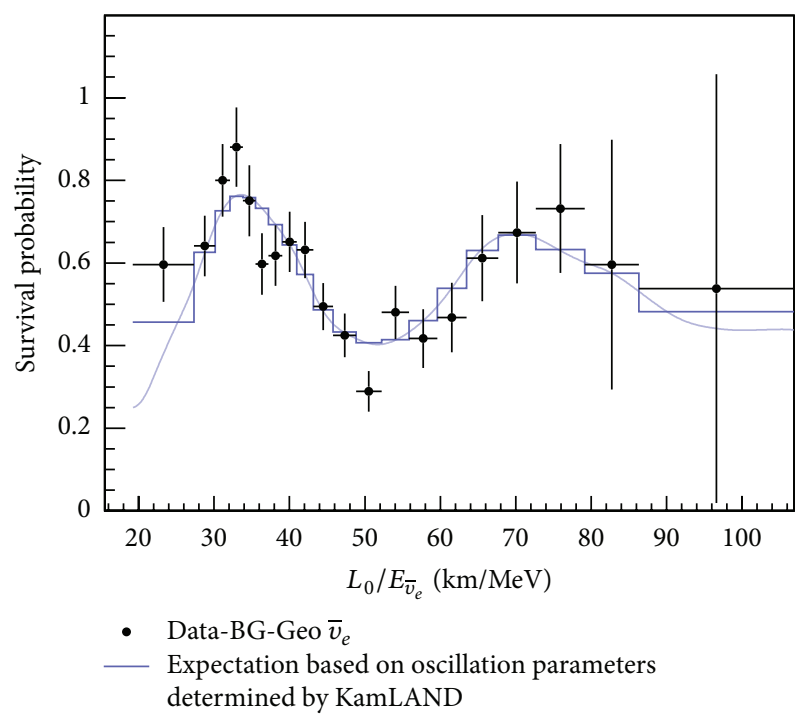

FIGURE 6: Ratio of the background and geo-neutrino-subtracted $\bar{v}_{e}$ spectrum to the expectation for no oscillation as a function of $L_{0} / E . L_{0}$ is the effective baseline taken as a flux-weighted average $\left(L_{0}=180 \mathrm{~km}\right)$. The energy bins are equal probability bins of the best fit including all backgrounds. The histogram and curve show the expectation accounting for the distances to the individual reactors, time-dependent flux variations, and efficiencies. The error bars are statistical only and do not include, for example, correlated systematic uncertainties in the energy scale. Taken from [40].

level of $\left.(6.8 \pm 1.5) 10^{-18} \mathrm{~g} / \mathrm{g}\right)$ and the ${ }^{85} \mathrm{Kr}$ contained in the scintillator that produces the rare decay sequence ${ }^{85} \mathrm{Kr} \rightarrow$ ${ }^{85 \mathrm{~m}} \mathrm{Rb}+e^{-}+\bar{v}_{e},{ }^{85 \mathrm{~m}} \mathrm{Rb} \rightarrow{ }^{85} \mathrm{Rb}+\gamma$. The total estimated systematic error was $8.5 \%$ [111], mainly determined by two sources, introducing an uncertainty of $6 \%$ each: the total uncertainty on the fiducial mass and the one on the response function. The best value for the interaction rate of the $0.862 \mathrm{MeV}^{7} \mathrm{Be}$ solar neutrinos was $49 \pm 3$ (stat.) \pm 4 (syst.) counts/(day $\cdot 100$ ton). This result excludes at the $4 \sigma \mathrm{CL}$ the no oscillation hypothesis for ${ }^{7} \mathrm{Be}$ solar neutrinos, which in the high metallicity SSM $[118,128]$ would imply $74 \pm 4$ counts/ (day 100 ton). The Borexino result is, instead, in a very good agreement with the predictions of the LMA oscillation solution: $48 \pm 4$ counts/(day. 100 ton).

In order to reduce the systematic uncertainties and to tune the reconstruction algorithm and Monte Carlo simulations, a calibration campaign was performed in 2009 introducing inside the Borexino detector several internal radio sources $\alpha$ 's, $\beta$ 's, $\gamma$ 's, and neutrons, at different energies and in hundreds of different positions, which were determined with a precision better than $2 \mathrm{~cm}$. The previous systematic error on ${ }^{7} \mathrm{Be}$ solar neutrino flux was estimated to be [111] at the level of $6 \%$ for both the fiducial volume and the energy scale (when thermal neutrons are captured by protons a 2.2 $\mathrm{MeV} \gamma$-ray is generated.) In the calibration campaign, the detector energy response was studied with eight $\gamma$ sources and $\mathrm{Am}-\mathrm{Be}$ neutron source and comparing the calibration data and Monte Carlo simulations at different energies within the solar neutrinos energy region. The energy scale uncertainty,

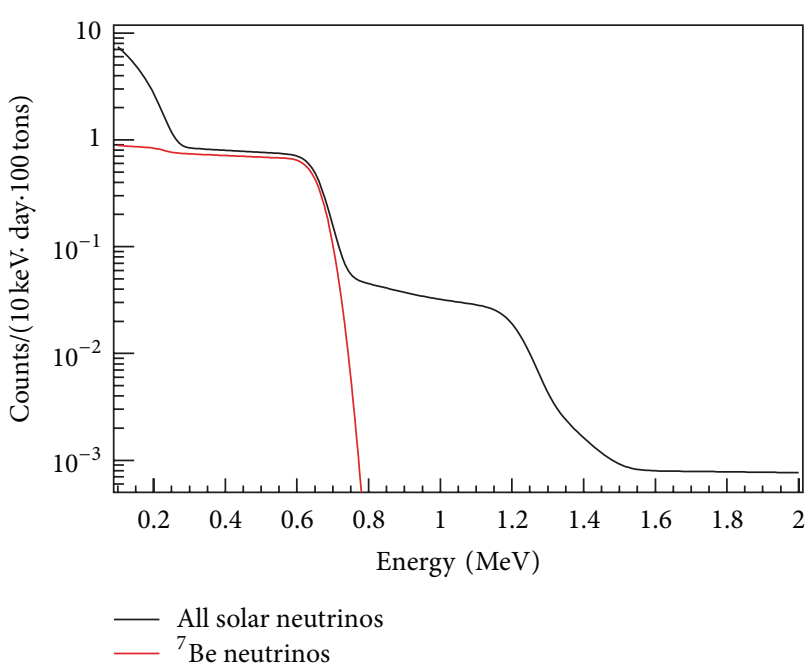

FIGURE 7: Neutrino spectra expected in Borexino (accounting for the detector's energy resolution). The upper line represents the neutrino signal rate in Borexino according to the most recent predictions of the standard solar model [118] including neutrino oscillations with the LMA-MSW parameters. The lower line illustrates the contribution due to ${ }^{7} \mathrm{Be}$ neutrinos. The $p p$ neutrinos contribute to the spectrum below $0.3 \mathrm{MeV}$ and the edge at $1.2 \mathrm{MeV}$ is due to pep neutrinos (from [119]).

obtained with these studies, was determined to be less than $1.5 \%$.

The inaccuracy of the position (reduced by means of studies with $\alpha$ and $\beta$ events) was less than $3 \mathrm{~cm}$, equivalent to a systematic error of $1.3 \%$ for the overall fiducial volume in the ${ }^{7} \mathrm{Be}$ solar neutrino energy region. The analyzed data set run from May 2007 to May 2010, with a fiducial exposure equivalent to 153.6 ton $\cdot$ year. In order to extract the ${ }^{7} \mathrm{Be}$ solar neutrino signal, the spectral fit was applied assuming all the intrinsic background components such as ${ }^{85} \mathrm{Kr},{ }^{210} \mathrm{Bi},{ }^{14} \mathrm{C}$, and ${ }^{11} \mathrm{C}$. The ${ }^{7} \mathrm{Be}$ solar neutrino rate was evaluated to be $46.0 \pm 1.5$ (stat) ${ }_{-1.6}^{+1.5}$ (syst) counts/day 100 ton [129]. Thanks to the calibration campaign, the systematic error was reduced to $2.7 \%$ and the total uncertainty to $4.3 \%$.

5.4. The pep and CNO Neutrinos Measurement in Borexino. In the SSM, due to the solar luminosity constraint and their intimate link to the $p p$ neutrinos $[43,56]$, the monoenergetic $1.44 \mathrm{MeV}$ pep neutrinos have one of the smallest uncertainties (1.2\%) [64]. For this reason, after the $p p$ neutrinos, they constitute the ideal probe to test SSM hypotheses. On the other hand, the detection of neutrinos within the CNObicycle is central to probe the solar core metallicity and contribute in this way to the solution of the solar metallicity problem $[64,130]$. Also, they are believed to fuel massive stars with mass greater than $\sim 1.2 \mathrm{M}_{\odot}$ during the main sequence evolution and also stars with lower masses in more advanced stages of evolution. The energy spectrum of neutrinos from the CNO-bicycle is the result of three continuous spectra with end point energies of $1.19 \mathrm{MeV}\left({ }^{13} \mathrm{~N}\right), 1.73 \mathrm{MeV}\left({ }^{15} \mathrm{O}\right)$, and 


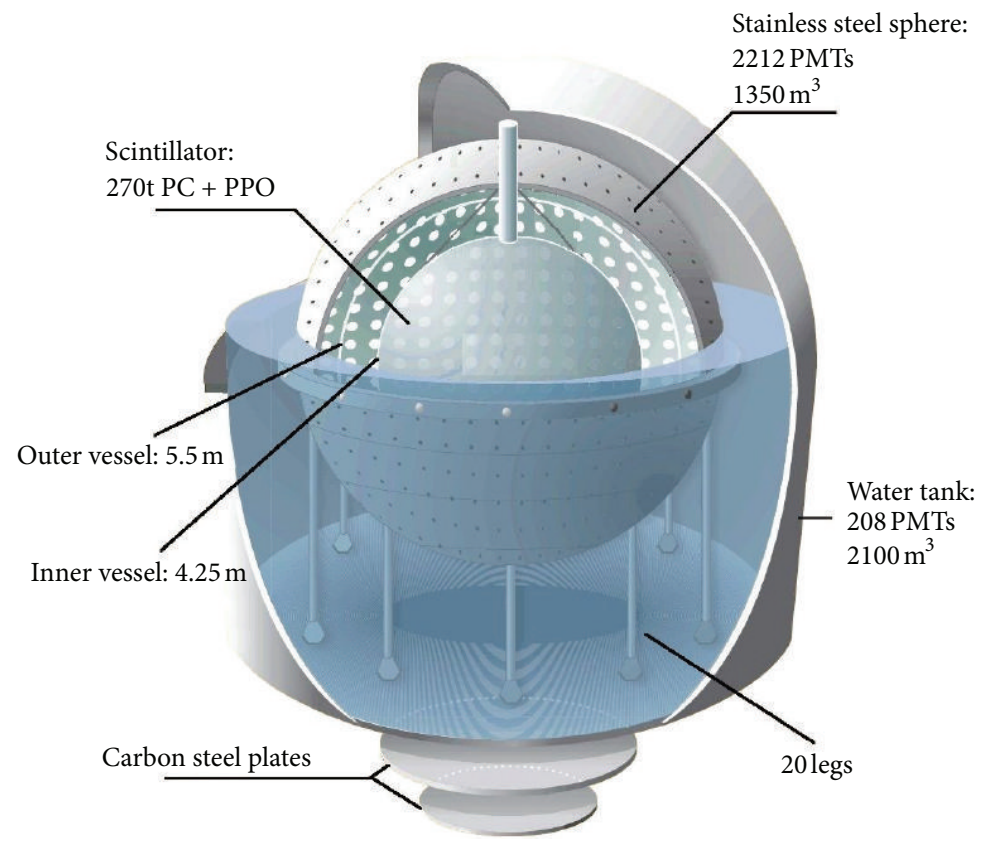

FIGURE 8: Schematic view of the Borexino detector.

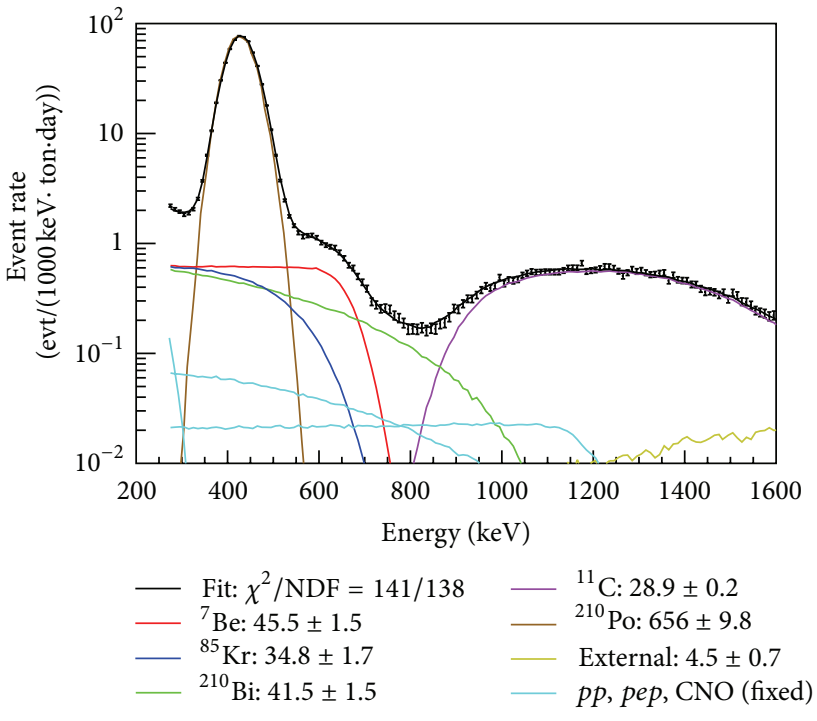

(a)

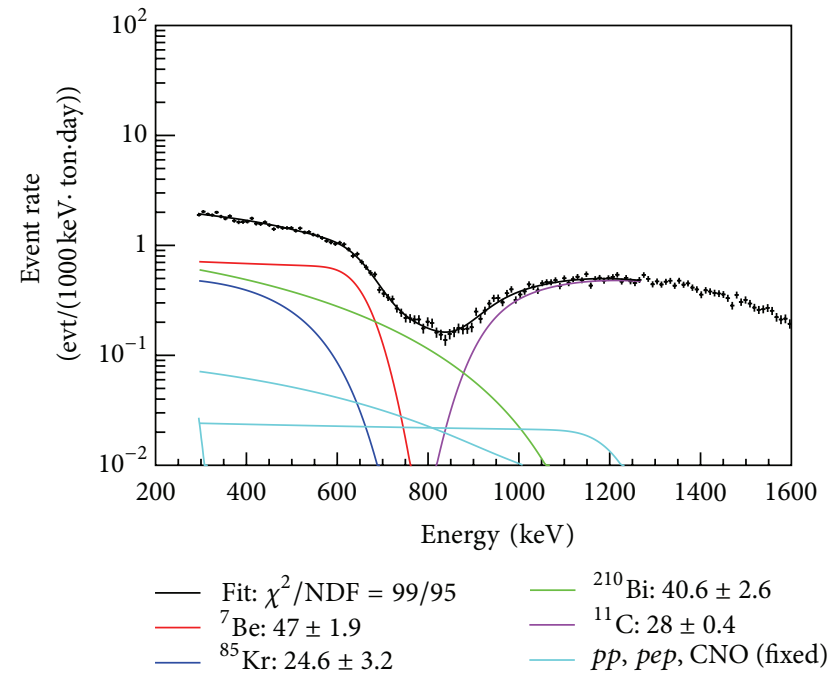

(b)

FIGURE 9: Examples of fitted spectra; the fit results in the legends have units [counts/(day·100 ton)]. (a): A Monte Carlo based fit over the energy region $270-1600 \mathrm{keV}$ to a spectrum from which some, but not all, of the $\alpha$ events have been removed using a PSA cut, and in which the event energies were estimated using the number of photons detected by the PMT array. (b): An analytic fit over the 290-1270 keV energy region to a spectrum obtained with statistical $\alpha$ subtraction and in which the event energies were estimated using the total charge collected by the PMT array. In all cases the fitted event rates refer to the total rate of each species, independently from the fit energy window (from [129]).

1.74 MeV $\left({ }^{17} \mathrm{~F}\right)$. Despite their relevance, until 2011 no pep and $\mathrm{CNO}$ neutrinos had been detected directly.

The electron recoil energy spectrum from pep neutrino interactions in Borexino is a Compton-like shoulder with end point of 1.22 MeV, as one can see from Figures 9 and 10, showing the pep and CNO contribution in Borexino.
As already mentioned, very low background levels [111, 119] are required to detect ${ }^{7} \mathrm{Be}$ neutrinos; the detection of pep and CNO neutrinos is even more challenging, as their expected interaction rates are $\sim 10$ times lower. The expected rate is on the order of a few counts per day in a 100ton target. To detect pep and CNO neutrinos, the Borexino 


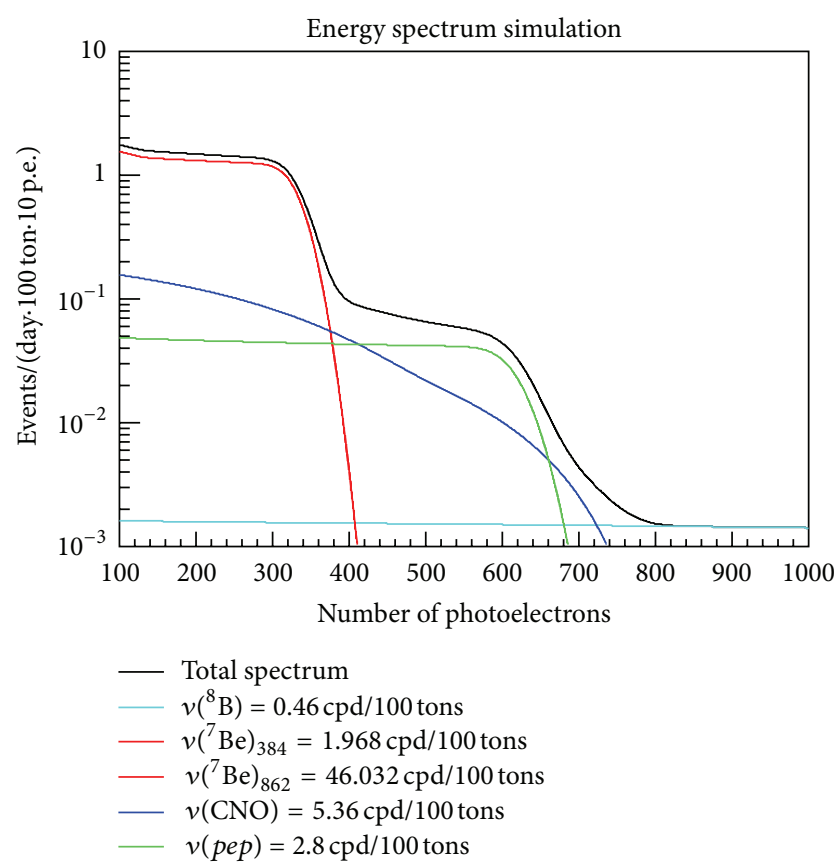

FIGURE 10: The neutrino-induced electron recoil spectra expected in Borexino. The total rates are those predicted by the latest $h i g h-Z$ solar model [64]. The pep and CNO neutrinos recoil spectra with end points in the region $1.2-1.5 \mathrm{MeV}$ are shown. Also the ${ }^{7} \mathrm{Be}$ neutrinos (measured in [129]), with a count rate about 10 times larger, are shown for comparison. Note that the variable on the $x$-axis is not directly the energy value. Taken from [131].

collaboration adopted a novel analysis procedure to suppress the dominant background in the $1-2 \mathrm{MeV}$ energy range, due to the cosmogenic $\beta^{+}$-emitter ${ }^{11} \mathrm{C}$ produced within the scintillator by muon interactions with ${ }^{12} \mathrm{C}$ nuclei. The muon flux crossing the Borexino detector, $\sim 4300 \mu /$ day, yields a ${ }^{11} \mathrm{C}$ production rate of $\sim 27$ counts/(day $\cdot 100$ ton) (In 95\% of the cases at least one free neutron is spalled in the ${ }^{11} \mathrm{C}$ production process [132], and then captured in the scintillator with a mean time of $255 \mu$ s [133].). This background can be reduced by performing a space and time veto following coincidences between signals from the muons and the cosmogenic neutrons $[134,135]$, discarding exposure that is more likely to contain ${ }^{11} \mathrm{C}$ due to the correlation between the parent muon, the neutron, and the subsequent ${ }^{11} \mathrm{C}$ decay (the three-fold coincidence, TFC). The TFC technique is based on the reconstructed track of the muon and the reconstructed position of the neutron-capture $\gamma$-ray [133]. The criteria of rejection were applied to obtain the best compromise between ${ }^{11} \mathrm{C}$ rejection and preservation of fiducial exposure, resulting in a ${ }^{11} \mathrm{C}$ rate of $(2.5 \pm 0.3)$ count per day and $(9 \pm 1) \%$ of the original rate, while preserving $48.5 \%$ of the initial exposure.

Figure 11 shows the resulting spectrum obtained with data collected between January 2008 and May 2010, corresponding to a fiducial exposure of 20409 ton- day [74]. Despite the TFC veto, the number of ${ }^{11} \mathrm{C}$ surviving events still constituted a significant background.

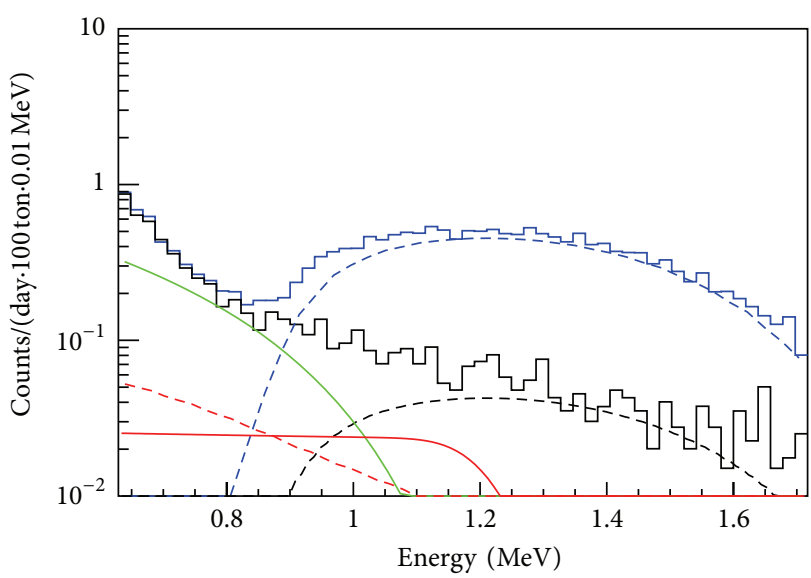

$$
\begin{aligned}
& - \text { Spectrum of events in FV }-{ }^{210} \mathrm{Bi} \text { rate }=55 \\
& \text { _- Spectrum after TFC veto _ - }{ }^{11} \mathrm{C} \text { rate }=2.5 \\
& --{ }^{11} \mathrm{C} \text { rate }=27 \quad---\mathrm{CNO} v \text { limit }=7.9 \\
& \text { pep } v \text { rate }=3.1 \quad \text { (95\% C.L.) }
\end{aligned}
$$

FIGURE 11: Energy spectra of the events in the FV before and after the TFC veto is applied. The solid and dashed blue lines show the data and estimated ${ }^{11} \mathrm{C}$ rate before any veto is applied. The solid black line shows the data after the procedure, in which the ${ }^{11} \mathrm{C}$ contribution (dashed) has been greatly suppressed. The next largest background, ${ }^{210} \mathrm{Bi}$, and the electron recoil spectra of the best estimate of the pep neutrino rate and of the upper limit of CNO neutrino rate are shown for reference. Rate values in the legend are quoted in counts/(day 100 ton) from [74].

To discriminate ${ }^{11} \mathrm{C} \beta^{+}$decays from neutrino-induced $e^{-}$ recoils and $\beta^{-}$decays, the pulse shape differences between $e^{-}$ and $e^{+}$interactions in organic liquid scintillators $[136,137]$ were exploited. In fact a small difference in the time distribution of the scintillation signal arises from the finite lifetime of orthopositronium as well as from the presence of annihilation $\gamma$-rays, which present a distributed, multisite event topology and a larger average ionization density than electron interactions. The Borexino collaboration employed an optimized pulse shape parameter using a boosted-decision-tree algorithm [138] and trained with a TFC-selected set of ${ }^{11} \mathrm{C}$ events $\left(e^{+}\right)$and ${ }^{214} \mathrm{Bi}$ events $\left(e^{-}\right)$selected by the fast ${ }^{214} \mathrm{Bi}-$ ${ }^{214}$ Po $\alpha$ - $\beta$ decay sequence (less than $2.8 \mathrm{~m}$ from the detector center and with a vertical position relative to the detector center between $-1.8 \mathrm{~m}$ and $2.2 \mathrm{~m}$.) In a work published in 2012 [74], the Borexino collaboration presented the results of an analysis based on a binned likelihood multivariate fit performed on the energy, pulse shape, and spatial distributions of selected scintillation events whose reconstructed position is within the fiducial volume.

The energy spectra and spatial distribution of the external $\gamma$-ray backgrounds have been obtained from a full, Geant4based Monte Carlo simulation and validated with calibration data from a high-activity ${ }^{228} \mathrm{Th}$ source [139] deployed in the outermost buffer region, outside the active volume. $\alpha$ events were removed from the energy spectrum by the statistical subtraction method [119]. In the energy region of interest of the fit procedure all background species whose rates were 


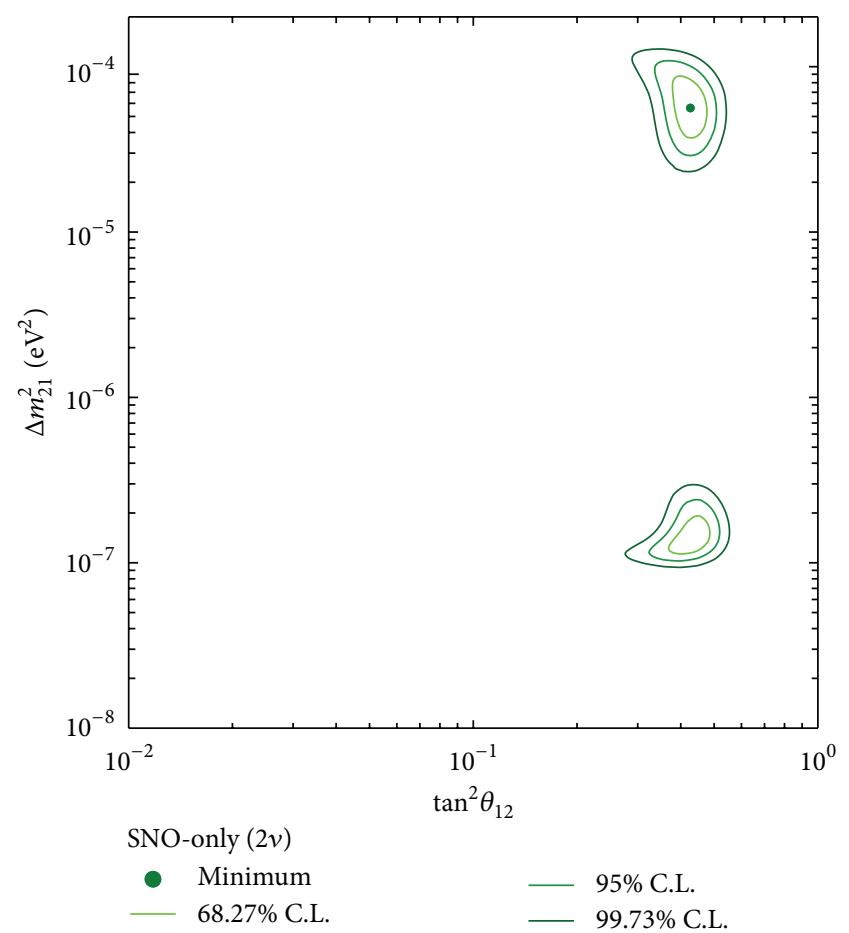

FIGURE 12: Two-flavor neutrino oscillation analysis contour using only SNO data (taken from [147]).

estimated to be less than $5 \%$ of the predicted rate from pep neutrinos have been excluded (Electron recoils from ${ }^{7} \mathrm{Be}$, pep, and $\mathrm{CNO}$ solar neutrinos, internal radioactive backgrounds ${ }^{210} \mathrm{Bi},{ }^{11} \mathrm{C},{ }^{10} \mathrm{C},{ }^{6} \mathrm{He},{ }^{40} \mathrm{~K},{ }^{85} \mathrm{Kr}$, and ${ }^{234 \mathrm{~m}} \mathrm{~Pa}$, and external $\gamma$-rays from ${ }^{208} \mathrm{Tl},{ }^{214} \mathrm{Bi}$, and ${ }^{40} \mathrm{~K}$.). All rates were constrained to positive values and thirteen species were left free in the fit. The rate of the radon daughter ${ }^{214} \mathrm{~Pb}$ was fixed using the measured rate of ${ }^{214} \mathrm{Bi}^{2}{ }^{214} \mathrm{Po}$ delayed coincidence events. The contribution from $P p$ solar neutrinos was fixed to the SSM assuming MSW-LMA [140-145] with $\tan ^{2} \theta_{12}=$ $0.47_{-0.04}^{+0.05}, \Delta m_{12}^{2}=(7.6 \pm 0.2) \cdot 10^{-5} \mathrm{eV}^{2}[146]$, and the contribution from ${ }^{8} \mathrm{~B}$ neutrinos to the rate from the measured flux $[112,147]$.

In Table 6, the results for the pep and CNO neutrino interaction rates are shown. The absence of a pep neutrino signal was rejected at $98 \% \mathrm{CL}$. Concerning the CNO neutrinos flux, its electron-recoil spectrum is similar to the spectral shape of ${ }^{210} \mathrm{Bi}$, but the last one is about 10 times greater; therefore, it has only been possible to provide an upper limit on the CNO neutrino interaction rate. The 95\% CL limit reported in Table 6 has been obtained from a likelihood ratio test with the $p p$ neutrino rate fixed to the SSM prediction [64] under the assumption of MSW-LMA, (2.80 \pm 0.04$)$ counts/(day · 100 ton).

\section{Phenomenological Analysis}

6.1. Status of the Determination of the Mixing Parameters in a 3-Flavor Analysis. Recently, the SNO collaboration performed a combined analysis of all the three working phases

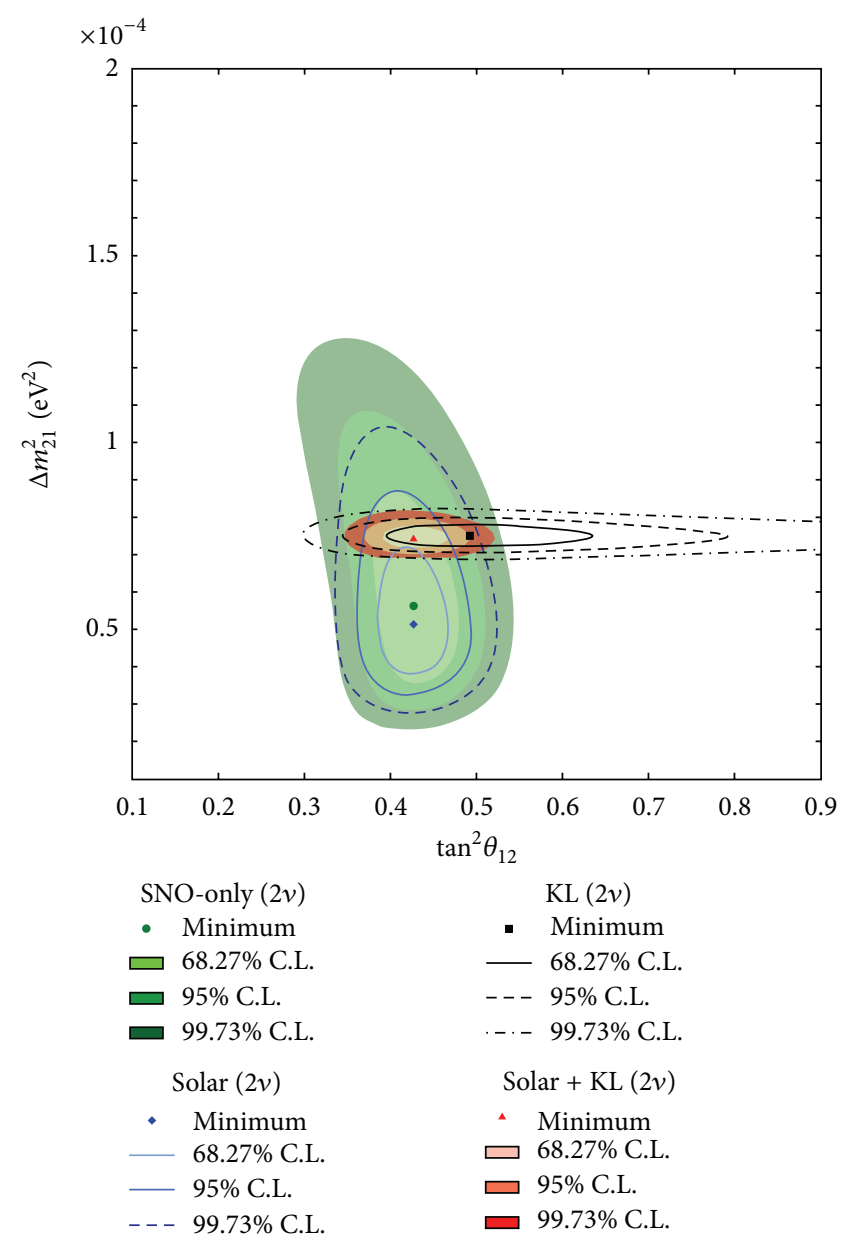

FIGURE 13: Two-flavor neutrino oscillation analysis contour using both solar neutrino and KamLAND results (taken from [147]).

of the experiment [147] based on a fit to Monte Carlo derived probability density functions (PDFs) for each of the possible signals and backgrounds, and also introduced a new way to parametrize the ${ }^{8} \mathrm{~B}$ neutrino signal. Figure 12 , reporting the results of the two-flavour (with the assumption $\theta_{13}=0$ ) SNO only analysis, shows the further improvement in the mixing parameters accuracy, but, at the same time, it confirms that the $\mathrm{SNO}$ results alone would not be sufficient to completely exclude the LOW solution.

This ambiguity was definitely removed, as shown in Figure 13 , by including in the analysis the results of all previous solar neutrino experiments [110, 114, 115, 150-153], the ${ }^{7} \mathrm{Be}$ solar neutrino rate measured by Borexino [129], the ${ }^{8} \mathrm{~B}$ neutrino spectra [154], and the KamLAND data [41] (The KamLAND data were obtained in a completely independent experiment and, therefore, the corresponding $\chi^{2}$ values, as functions of the mixing parameters, were directly summed to the $\chi^{2}$ values computed by direct solar neutrino analysis.).

The higher values of $\Delta m_{12}^{2}$ in the LMA region were excluded, together with the full LOW solution, thanks mainly to the large discrimination power of KamLAND. This experiment, however, did not contribute significantly to improve the mixing angle determination and the accuracy on this 

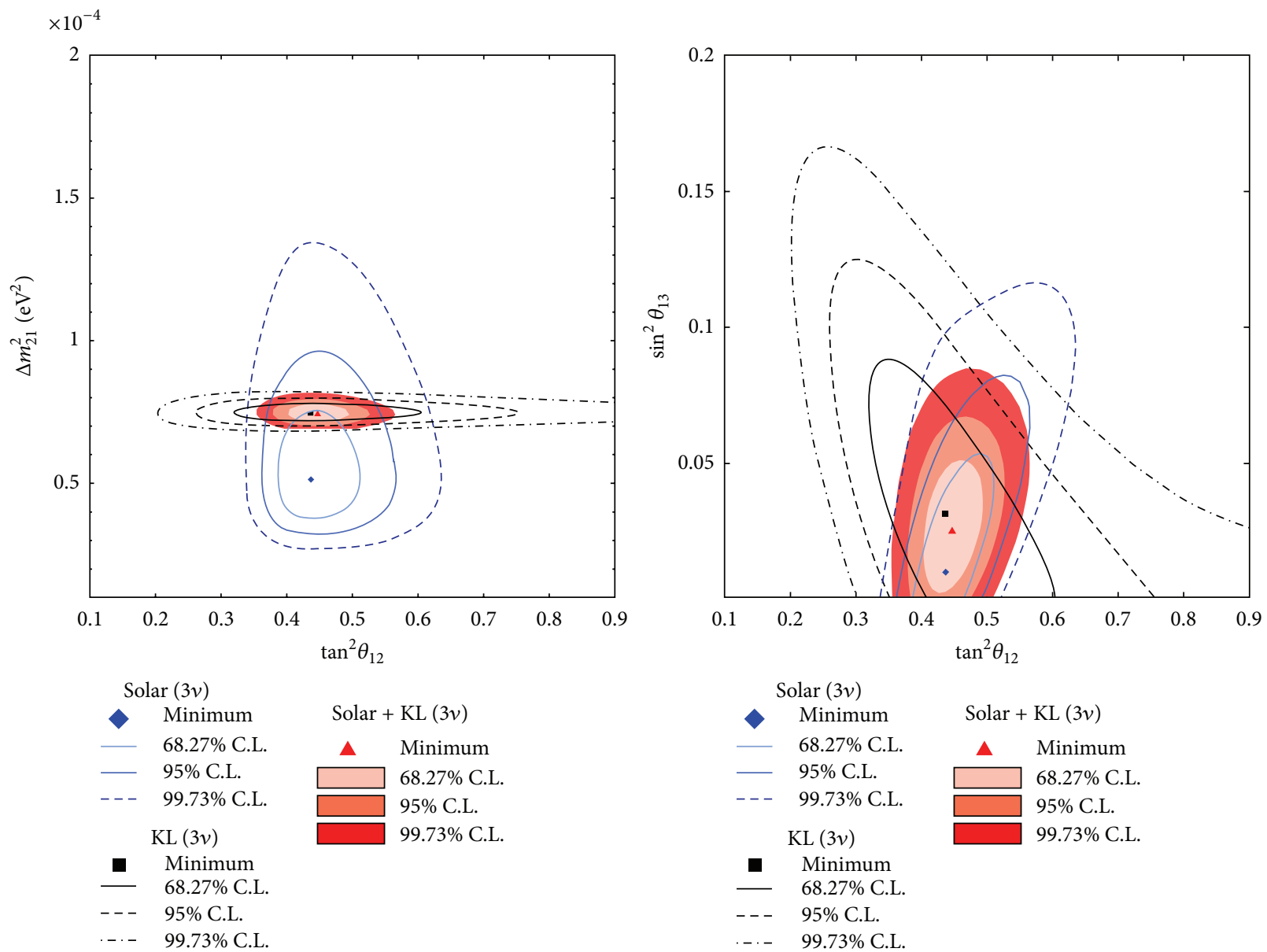

FIGURE 14: Three-flavor neutrino oscillation analysis contour using both solar neutrino and KamLAND results. Taken from [147].

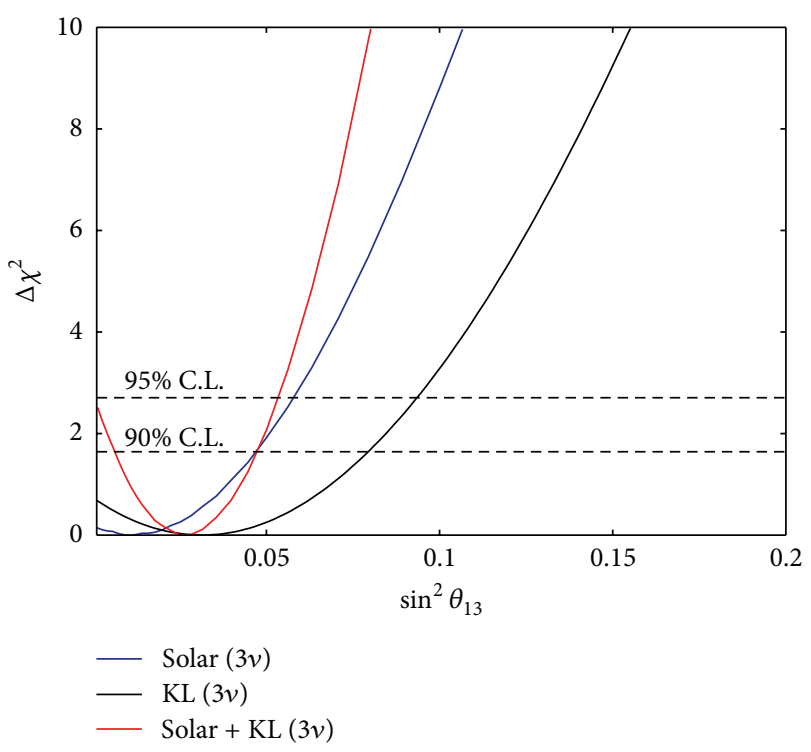

FIGURE 15: Projections of the three-flavor neutrino oscillation parameters. The horizontal lines represent the $\Delta \chi^{2}$ for a particular confidence level. Taken from [147]. parameter remained quite high. The results of the two-flavor analysis are reported in Table 7 (taken from [147]).

The slight tension between the solar neutrino experiments and KamLAND was significantly reduced by extending the analysis to the 3 flavor oscillation case as shown in Figure 14, from which it is clear that the best global fit is obtained for values of $\theta_{13}$ different from zero.

A detailed analysis of the $\chi^{2}$ behavior proved also that the combination of solar experiments and KamLAND enables the significant improve of the discriminating power on the $\theta_{13}$ mixing parameter (see Figure 15 and Table 8 ).

The indication in favor of $\theta_{13}$ being different from zero was in agreement with the recent results from the longbaseline experiments T2 K [155] and MINOS [156], and with the combined analysis performed in [157], including also the atmospheric neutrino and the CHOOZ [158] data. Moreover, the validity of this hint has been corroborated by the data obtained this year by the short baseline neutrino reactor experiments [159-162], which established that $\theta_{13}>0$ at about $5 \sigma$ (and even more in the Daya Bay case [163]). These experiments found values of $\sin ^{2} \theta_{13}$ centered between 0.020 and 0.030 , very promising results for future experiments 
TABLE 6: Best estimates for the pep and CNO solar neutrino interaction rates. For the results in the last two columns both statistical and systematic uncertainties are considered. Total fluxes have been obtained assuming MSW-LMA and using the scattering cross-sections from $[146,148,149]$ and a scintillator $e^{-}$density of $(3.307 \pm 0.003) \cdot 10^{29} \operatorname{ton}^{-1}$. The last column gives the ratio between our measurement and the high-Z (GS98) SSM [64]. Table taken from [74].

\begin{tabular}{lccc}
\hline$v$ & $\begin{array}{c}\text { Interaction rate } \\
[\text { counts/(day } \cdot 100 \text { ton })]\end{array}$ & $\begin{array}{c}\text { Solar- } v \text { flux } \\
{\left[10^{8} \mathrm{~cm}^{-2} \mathrm{~s}^{-1}\right]}\end{array}$ & $\begin{array}{c}\text { Data/SSM } \\
\text { ratio }\end{array}$ \\
\hline pep & $3.1 \pm 0.6_{\text {stat }} \pm 0.3_{\text {syst }}$ & $1.6 \pm 0.3$ & $1.1 \pm 0.2$ \\
CNO & $<7.9\left(<7.1_{\text {stat only }}\right)$ & $<7.7$ & $<1.5$ \\
\hline
\end{tabular}

TABLE 7: Best-fit neutrino oscillation parameters from a two-flavor neutrino oscillation analysis. Uncertainties listed are $1 \sigma$ after the $\chi^{2}$ was minimized with respect to all other parameters (taken from [147]).

\begin{tabular}{lccr}
\hline Analysis & $\tan ^{2} \theta_{12}$ & $\Delta m_{21}^{2}\left[\mathrm{eV}^{2}\right]$ & $\chi^{2} / \mathrm{NDF}$ \\
\hline SNO only (LMA) & $0.427_{-0.029}^{+0.033}$ & $5.62_{-1.36}^{+1.92} \times 10^{-5}$ & $1.39 / 3$ \\
SNO only (LOW) & $0.427_{-0.035}^{+0.043}$ & $1.35_{-0.14}^{+0.35} \times 10^{-7}$ & $1.41 / 3$ \\
Solar & $0.427_{-0.028}^{+0.028}$ & $5.13_{-0.96}^{+1.29} \times 10^{-5}$ & $108.07 / 129$ \\
Solar + KamLAND & $0.427_{-0.024}^{+0.027}$ & $7.46_{-0.19}^{+0.20} \times 10^{-5}$ & \\
\hline
\end{tabular}

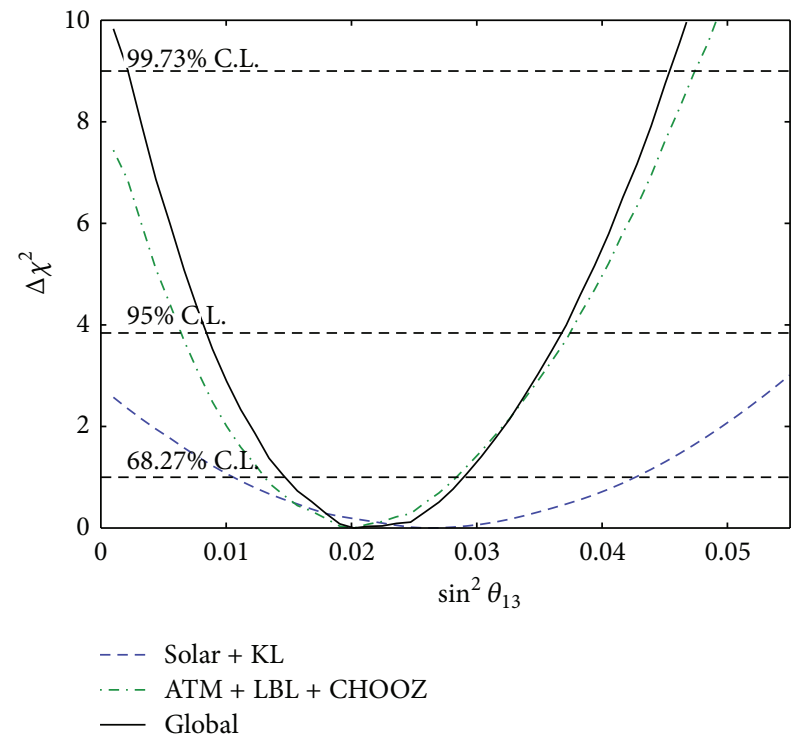

FIGURE 16: Projection over $\sin ^{2} \theta_{13}$ combining the projections obtained by analyzing data from all neutrino sources. The data from atmospheric, short-baseline experiments, and long-baseline experiments $(\mathrm{ATM}+\mathrm{LBL}+\mathrm{CHOOZ})$ was determined from Figure 2 (left panel) in [157] which already includes the latest T2 K [155] and MINOS [156] results.

looking for leptonic CP violation [164]. The impact and the possible consequences of these recent results have been discussed, among the others, in the following papers [164167]. The different accuracy that can be reached in the determination of the mixing angle between the first and third generation, according to the different kind of neutrino experiments included in the analysis, is represented in Figure 16.

The combined analysis of the different SNO phases was also very useful to obtain a precise determination of the ${ }^{8} \mathrm{~B}$ solar neutrino flux, $\Phi_{8} \mathrm{~B}=5.25 \pm 0.16(\text { stat })_{-0.13}^{+0.11}($ syst $) \times$

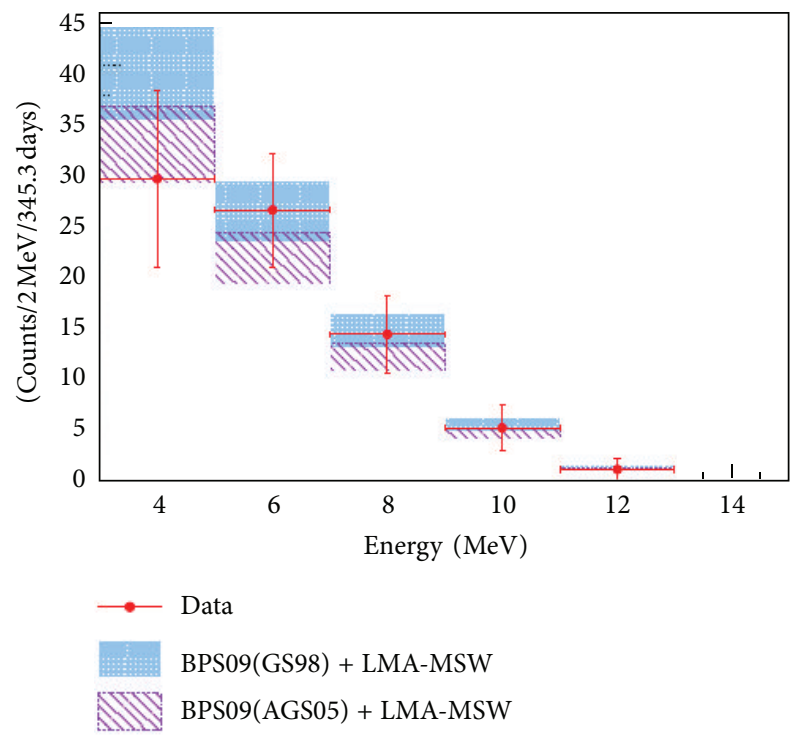

FIGURE 17: In this picture, taken from [154], the final energy spectrum derived from Borexino's analysis (red points) is represented and compared with the Monte Carlo predictions (blue and purple bands) obtained using the MSW-LMA neutrino oscillation model and the standard solar models in the versions corresponding, respectively, to the high metallicity (BPS09(GS98)) and the low metallicity (BPS09 (AGS05)) cases.

$10^{6} \mathrm{~cm}^{-2} \mathrm{~s}^{-1}$, with an important reduction of the systematic uncertainty. This result was consistent with, but more precise than, both the high-Z BPS09 (GS), $\Phi=(5.88 \pm 0.65) \times$ $10^{6} \mathrm{~cm}^{-2} \mathrm{~s}^{-1}$, and low-Z BPS09(AGSS09), $\Phi=(4.85 \pm 0.58) \times$ $10^{6} \mathrm{~cm}^{-2} \mathrm{~s}^{-1}$, and solar model predictions [72].

The combination of the LETA analysis by the SNO collaboration [112] and of the Borexino measurements [154] made possible a detailed study of the low energy part of the ${ }^{8} \mathrm{~B}$ solar neutrino spectrum. Even if characterized by a larger uncertainty (mainly due to a more limited statistics), Borexino 
TABLE 8: Best-fit neutrino oscillation parameters from a three-flavor neutrino oscillation analysis. Uncertainties listed are $\pm 1 \sigma$ after the $\chi^{2}$ was minimized with respect to all other parameters. The global analysis includes Solar + KL + ATM + LBL + CHOOZ.

\begin{tabular}{lccc}
\hline Analysis & $\tan ^{2} \theta_{12}^{2}$ & $\Delta m_{12}^{2}\left[\mathrm{eV}^{2}\right]$ & $\sin ^{2} \theta_{13} \times 10^{-2}$ \\
\hline Solar & $0.436_{-0.036}^{+0.048}$ & $5.13_{-0.98}^{+1.49} \times 10^{-5}$ & $<5.8(95 \%$ C.L.) \\
Solar + KL & $0.446_{-0.029}^{+0.030}$ & $7.41_{-0.19}^{+0.21} \times 10^{-5}$ & $2.5_{-1.5}^{+1.8}$ \\
& & & $<5.3(95 \%$ C.L.) \\
Global & & & $2.02_{-0.55}^{+0.88}$ \\
\hline
\end{tabular}

data confirm the LETA indication of low energy data points lower than the theoretical expectations based on matter enhanced oscillation and solar models as shown in Figure 17 (taken from [154]). These results agreed also with the SuperKamiokande observation [110] of flat spectrum, consistent with the undistorted spectrum hypothesis. The emergence of this slight tension between theory and experiments seems to indicate the presence of new subdominant effects and also suggests the possibility of nonstandard neutrino interactions (like those studied in [168]) or the mixing with a very light sterile neutrino [169]. Future solar neutrino experiments, like $\mathrm{SNO}+$, could shed more light on this subject, by performing precision measurements of lower energies solar neutrinos (like the pep neutrinos).

6.2. Free Flux Analyses. The increasing data of solar neutrinos allow to independently test the astrophysics of the solar interior and the physics of neutrino propagation. The analysis discussed in previous sections can be modified by also varying the solar neutrino fluxes in order to accommodate all neutrino data, while all the functional dependences are maintained as predicted by the standard model dependences. A key step in this kind of analysis is the imposition of the luminosity constraint $[170,171]$, which implements in a global way for the Sun the constraint of conservation of energy for nuclear fusion among light elements. Each neutrino flux is associated with a specific amount of energy released to the star and therefore a particular linear combination of the solar neutrino fluxes is equal to the solar luminosity (in appropriate units). One can write the luminosity constraint as

$$
\frac{L_{\odot}}{4 \pi(\text { A.U. })^{2}}=\sum_{i} \alpha_{i} \Phi_{i},
$$

where $L_{\odot}$ is the solar luminosity measured at the Earth's surface, $1 \mathrm{AU}$ is the average Earth-Sun distance, and the coefficient $\alpha_{i}$ is the amount of energy provided to the star by nuclear fusion reactions associated with each of the important solar neutrino fluxes, $\Phi_{i}$. The coefficients $\alpha_{i}$ are calculated accurately in [171].

The model independent determination of the solar neutrino fluxes $[172,173]$ shows that present solar neutrino data leads to accurate results for four fluxes and also the correlations between them. This information allows for a consistent global comparison of SSM fluxes with the inferred fluxes by neutrino data. Present data lead to the values for the inferred solar neutrino fluxes reported in the fourth column (labelled as "Solar") of Table 4 in Section 3. The precision of the ${ }^{7} \mathrm{Be}$ and ${ }^{8} \mathrm{~B}$ neutrino fluxes is driven by the Borexino and $\mathrm{SNO}$
(SK) neutrino experiments, while the precision of the $p p$ and pep neutrino fluxes mainly comes by the imposition of the luminosity constraint. The neutrino data directly demonstrates that the Sun shines by the $p p$ chain. The CNO cycle only contributes to the total luminosity at the percent level.

The reader may wonder how much these inferences are affected by the luminosity constraint. The idea that the Sun shines because of nuclear fusion reactions can be tested accurately by comparing the observed photon luminosity of the Sun with the luminosity inferred from measurements of solar neutrino fluxes. Moreover, this same comparison will test a basic result of the standard solar model, namely, that the Sun is in a quasi-steady state in which the current energy generation in the interior equals the current luminosity at the solar surface. The free flux analysis, without imposing luminosity constraint, permits an estimation of the solar luminosity inferred by neutrino data, which agrees with the directly measured one within $15 \%(1 \sigma)$.

\section{Future Solar Neutrino Experiments}

7.1. The Near Future: Improvement of pep Measurements and CNO Detection. In the last decades, the intensive study of ${ }^{8} \mathrm{~B}$ and, more recently, ${ }^{7} \mathrm{Be}$ solar neutrinos made possible fundamental steps forward in the solution of the solar neutrino puzzle and the determination of the neutrino mixing parameters. Nevertheless, many key features of the oscillation models (like the transition between the vacuum dominated sub-MeV region and the spectral region between 1 and $3 \mathrm{MeV}$, where matter effects become relevant) still have to be tested or verified with better accuracy and precision (see Figure 18, taken from [174]).

The apparent partial deficit of events in the low energy part of the ${ }^{8} \mathrm{~B}$ spectrum suggested the introduction of new theoretical models (as discussed in Section 6). Also for these reasons, the experimental efforts in the last years focused on the detection of neutrinos of ever decreasing energies, to fully confirm the validity of the MSW-LMA solution and verify the fluxes predicted by SSMs, discriminating between different versions of these models. The fluxes of the medium and high energy neutrinos of the $p p$ chains $\left({ }^{7} \mathrm{Be},{ }^{8} \mathrm{~B}\right.$, and hep) are predicted with quite large uncertainties, mainly due to the uncertainties in nuclear cross-sections and solar opacity (Table 5). The $p p$ and pep fluxes, instead, are strongly correlated between themselves and their values are predicted with the highest precision because SSMs predict that $p p$ chain reactions are responsible for more than $99 \%$ of the 


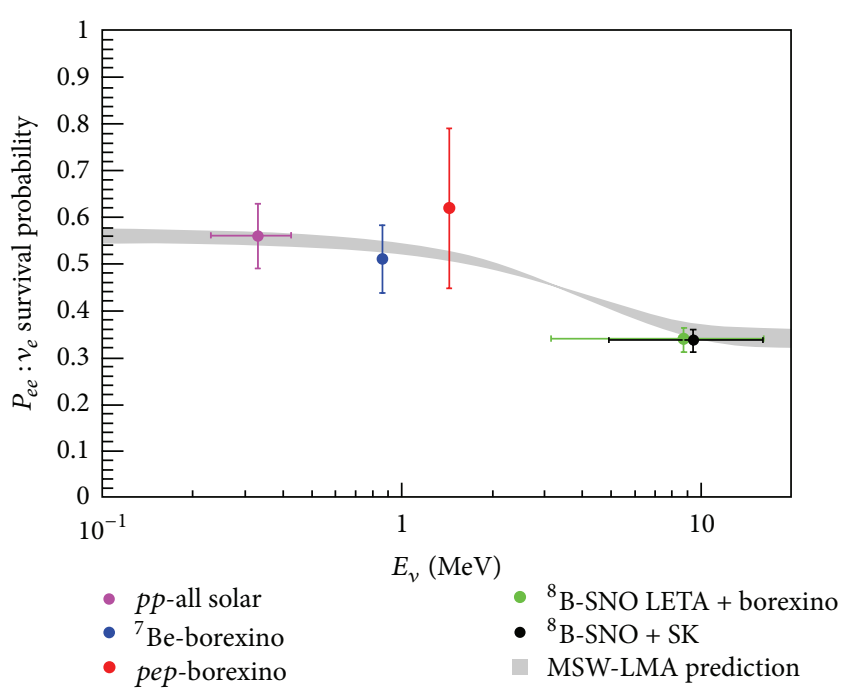

FIGURE 18: The $v_{e}$ survival probability is represented as a function of neu-trino energy. The gray band represent the MSW-LMA prediction. The higher survival probability region at low energies is where vacuum-dominated oscillations occur. As the neutrino energy increases, matter effects become important and the lower survival probability at high energies is due to matter-enhanced oscillations. The reported data correspond to solar neutrino flux measurements performed by different experiments. Taken from [174].

energy powering the Sun [80]. Therefore, the measurements of these components would be the most stringent test of the SSM. The tight correlation between pep and $p p$ neutrinos is theoretically well established and, therefore, even in the pessimistic hypothesis that $p p$ neutrinos could not be measured with the desired accuracy, a significant improvement in the pep neutrinos measurement with respect to data presently available would make it possible to reduce significantly the $15 \%$ indetermination on the solar luminosity (see Section 6.2) and to test indirectly the SSM's predictions that almost 100\% of solar energy is produced by nuclear burning.

As already mentioned, water Čerenkov detectors, which played a fundamental role in the solution of the solar neutrino problem, are characterized by a low photon yield [175, 176] and therefore can detect only the higher part of the spectrum (hep and ${ }^{8} \mathrm{~B}$ neutrinos with a threshold around $3.5 \mathrm{MeV})$. The radiochemical experiments $[153,177]$ are limited, instead, by their ability to measure only the integrated neutrino rate above the charge-current interaction threshold (down to $0.23 \mathrm{MeV}$ for the Gallium experiments), without the possibility to discriminate between the different spectrum components. Therefore, an important contribution should come from the present and future organic liquid scintillator detectors, planned to perform low energy solar neutrino spectroscopy. To reach this goal, they will take advantage from the high values of light yield (about $10^{4}$ photons per $\mathrm{MeV}$ of deposited energy) and from the possibility to assemble very large masses of high purity materials. The excellent levels of radiopurity, reached, for instance, at Borexino, and the typical geometry of these detectors (which are unsegmented and can be easily adapted to the definition of a fiducial volume) are fundamental to reduce the impact of the background, that is so critical due to the feebleness of the low energy signal.

In the near future, significant contributions are expected from Borexino and SNO+ [178] experiments (the main problem still surviving seems to be the reduction of ${ }^{210} \mathrm{~Pb}$.) Borexino has already proved its importance in this kind of analysis performing the first measurements of pep and $\mathrm{CNO}$ neutrinos (even if the level accuracy is not yet the desired one) and further reducing, with the purification campaign started since July 2010, the level of contamination from almost all of the main radioactive background sources. The purification efforts are still ongoing and should make possible a further improvement on the accuracy of the signal extraction. Also, a novel technique [179] would allow to determine the ${ }^{210} \mathrm{Bi}$ background. Preliminary calculations show that the $\mathrm{CNO}$ signal could be extracted from the ${ }^{210} \mathrm{Bi}$ background with one year of data taking. The $\mathrm{SNO}+$ experiment, that should start taking data soon in the SNOLAB, should take advantage from the location (about two times deeper underground than the Gran Sasso laboratory), with the consequent lower muon flux and a strongly reduced ${ }^{11} \mathrm{C}$ rate. Moreover, thanks to the detector mass (about three times larger than in Borexino), it should be able to reach a higher counting rate. This could determine a fundamental improvement at least in the case of the pep neutrino measurement, where a $5 \%$ uncertainty is expected, to a level that should make possible a significant test of the MSW transition region.

In the more optimistic scenarios, it may be also possible to attack the main problem of measuring the lowest energy parts of the solar neutrino spectrum, that is, the $p p$ neutrinos and the $0.38 \mathrm{MeV}$ Berillium line. In any case, the presence in organic scintillators of an intrinsic ${ }^{14} \mathrm{C}$ background will make this very low energy measurements an extremely hard task and they may require the introduction of new techniques, like the ones we are going to describe in the next subsections.

7.2. The Far Future: Experimental Challenges. The challenge for all future experiments aimed at measuring the low energy part of solar neutrino spectrum is that of assembling experimental devices with low energy thresholds suitable to detect a low rate signal in a region characterized by different potential sources of radioactive background. This difficult experimental task is common also to the experiments looking for neutrinoless double $\beta$ decay or for dark matter signals (search for signatures of WIMPs, a stable or long-lived weakly interacting elementary particle, produced in the early Universe, whose existence is predicted in extensions of the standard model). In fact, some of the solar neutrino experiments planned for the future are multipurpose experiments designed also for the other above-quoted topics.

They are all characterized by a very large detector target mass and by the need to reach very high levels of radiopurity. The common feature is that of using scintillator detectors, but they differ for the chosen active scintillator material, which can vary from traditional organic scintillators (developed 
with the use of innovative technological devices) to new materials, like the noble gases.

7.2.1. Noble Liquid Detectors: CLEAN and XMASS. One of the possible future frontiers is the idea to use scintillation detectors with liquid noble gases, like xenon, argon, and neon. These materials have the advantage of being relatively inexpensive, easy to obtain, and dense and it is not too difficult to build large homogeneous detectors of this kind; moreover, they can be quite easily purified, offer very high scintillation yields (about 30-40 photons $/ \mathrm{keV}$ ), and do not absorb their own scintillation light.

A first example is offered by the CLEAN/DEAP family, a series of detectors based entirely on scintillation in liquid neon (LNe) and liquid argon (LAr). They have been realized using a scaleable technology in order to reach increasing sensitivities in the different prototypes realized and installed in the SNOLAB (Pico-CLEAN, Micro-CLEAN, DEAP-I, Mini-CLEAN and CLEAN/DEAP) with the aim to search for dark matter and to perform (through the analysis of elastic neutrino-electron and neutrino-nucleus scattering) a realtime measurement of the $p p$ solar neutrino flux. The final detector CLEAN (cryogenic low energy astrophysics with noble gases) [180] (see Figure 19) will be made by a stainless steel tank, of about 6 meters of diameter, filled with 135 metric tons of cryogenic liquid neon; only the central part of it, surrounded isotropically by a series of photomultipliers, will constitute the detector fiducial volume. An external tank of water, 10 metres wide and 12 metres high, will act as $\gamma$-ray shielding, neutron shielding, and muon veto. According to Monte Carlo simulations, there should be a production of 15000 photons/MeV and it should be possible to reach a $100 \%$ photon wavelength shifter efficiency and a statistical uncertainty on the $p p$ measurements of the order of $1 \%$.

A precise measurement of the $p p$ component and of the ratio between $p p$ and ${ }^{7} \mathrm{Be}$ fluxes would be essential to test the predictions of SSMs. A high accuracy on the $p p$ neutrino flux would also make possible a better determination of the $\theta_{12}$ mixing angle, which, complemented with the results from previous solar neutrino experiments and from KamLAND (essential for the $\Delta m_{12}^{2}$ measurement), would be fundamental to test the consistency of the LMA solution also in the region of transition between vacuum dominated and matter enhanced oscillations. Finally, CLEAN could in principle try to measure also the $\mathrm{CNO}$ neutrino flux, through the analysis of neutrino spectrum from 0.7 to $1.0 \mathrm{MeV}$, with an estimated accuracy between $10 \%$ and $15 \%$.

An interesting alternative to the use of neon is offered by liquid xenon scintillator detectors [181], which take advantage of the fact that among liquid rare gases xenon has the highest stopping power for penetrating radiation (thanks to its high atomic number, $A \simeq 131$, and density, $\rho=3 \mathrm{~g} / \mathrm{cm}^{3}$ ) and also the highest ionization and scintillation yield. The technological improvements of the last twenty to thirty years made possible significant improvements in the cooling and purification techniques of this kind of detectors and in the possibility of assembling large mass detectors, of the order of some

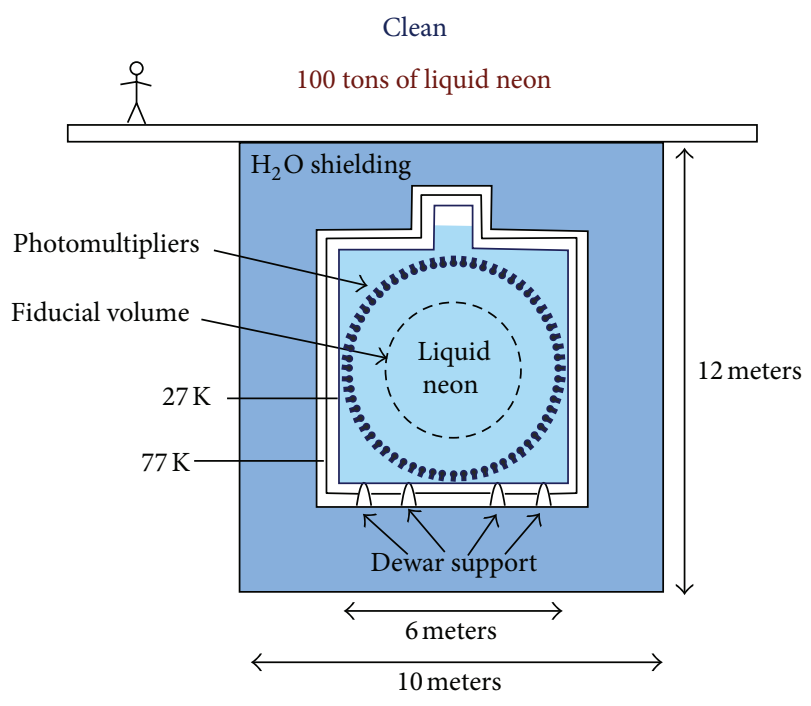

FIGURE 19: Scheme of the CLEAN detector. Taken from [180].

tons (like in the case of MEG [182] experiment, studying the $\mu \rightarrow e \gamma$ decay).

The XMASS experiment (see Figure 20) is a multipurpose low background and low energy threshold experiment that will use a large massive liquid xenon detector and has been designed to look for WIMPs (dark matter candidates), search for neutrinoless double $\beta$ decay, and study the $p p$ and the ${ }^{7} \mathrm{Be}$ solar neutrinos. After two preliminary phases, during which smaller prototypes have been realized and installed in the Kamioka mine [183], and the first data on double beta decay and dark matter have been taken, the full XMASS detector (that will measure also solar neutrinos) will have a total mass of 20 metric tons, with a fiducial volume of 10 metric tons. Special efforts are required mainly to lower the background, by reducing the radioactive contamination in the parts used for detector construction (with special attention to the photomultipliers and the copper material used for PMT holder), constructing a larger pure water active shield (for muons and mainly neutrons and $\gamma$ rays), and, above all, developing a distillation system for xenon in order to reduce the contamination by ${ }^{85} \mathrm{Kr}$, the major source of radioactive background inside the detector.

Another interesting experimental project based on the noble gases liquid scintillator technique is that of DARWIN (dark matter wimp search with noble liquids) [185], which brings together different European and US research groups working on existing experiments and on the study for a future multiton scale LAr and LXe dark matter search facility in Europe. The main goal of the experiment is to look for a WIMP signal and to demonstrate its dark matter nature, taking advantage from the fact of performing the measurement with multiple different targets operating under similar conditions. In this way, it should be possible to estimate the dependence of the rate with the target material and, therefore, to better determine the WIMP candidate mass and to distinguish between spin independent and spin dependent 


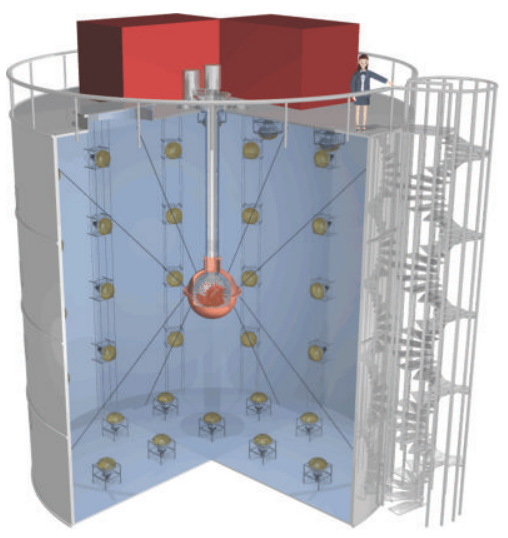

(a)

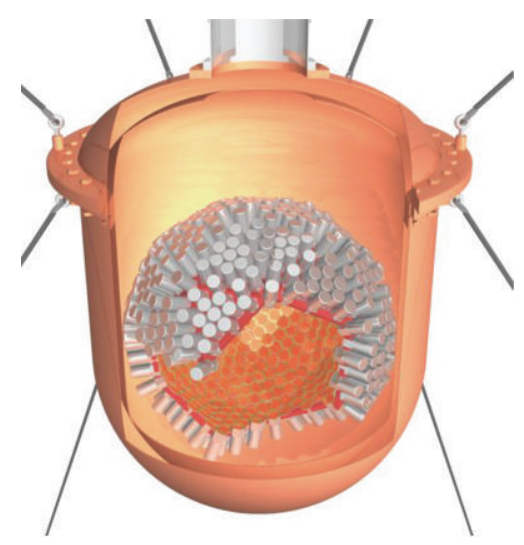

(b)

FIGURE 20: Schematic view of the full XMASS facility (a) and details of the inner detector (b) from which one can see the particular configuration of the hexagonal photomultiplier tubes. Taken from $[183,184]$.

couplings. The energy region of the nuclear recoil spectrum, below $200 \mathrm{keV}$, that should be investigated by this future experiment is of particular interest also for the study of the $p p$ solar neutrinos and, in fact, the elastic scattering on electrons by the low energy component of the neutrino spectrum would be one of the main background sources for WIMP searches in liquid xenon detectors, as shown in Figure 21.

DARWIN officially started in 2010; a technical design study should be ready in Spring 2013 and the start of the first physics run is expected by mid- 2017.

7.2.2. Multikiloton Scale Liquid Scintillators: Example LENA. The Borexino experiment demonstrated the great potential of the liquid-scintillator technique for the detection of low energy solar neutrinos. Thanks to this experience, a nextgeneration neutrino detector has been proposed: LENA (low energy neutrino astronomy) [186]. LENA is a multipurpose detector aiming to study supernova neutrinos, diffuse supernova neutrino background, proton decay, atmospheric neutrinos, long-baseline neutrino beams, geoneutrinos, and, last but not least, solar neutrinos. The LENA project foresees a cylindrical detector with a diameter of $30 \mathrm{~m}$ and a length of

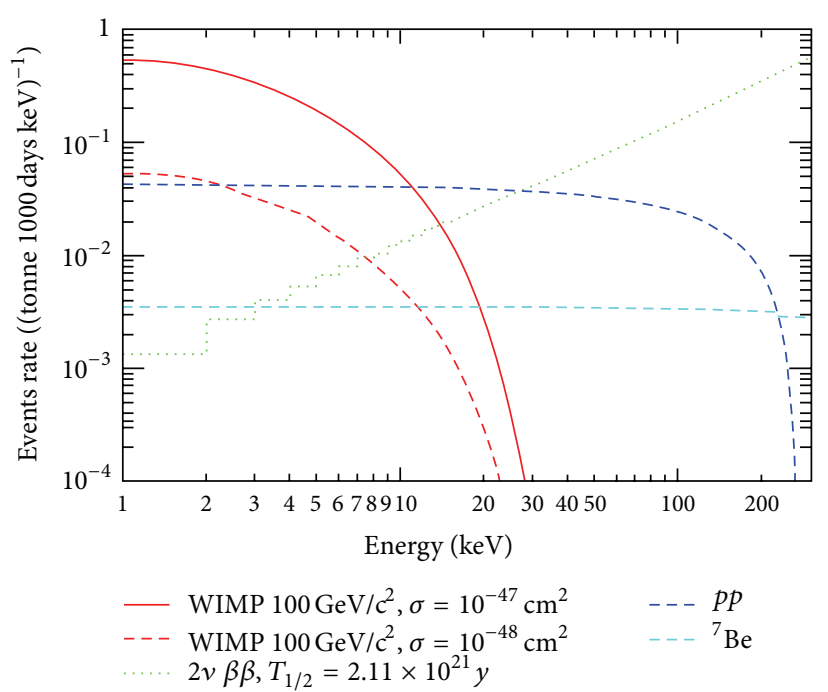

FIGURE 21: Expected nuclear recoil spectrum from WIMP scatters in LXe for a spin-independent WIMP-nucleon cross-section of $10^{-47} \mathrm{~cm}^{2}$ (red solid) and $10^{-48} \mathrm{~cm}^{2}$ (red dashed) and a WIMP mass of $100 \mathrm{GeV} / \mathrm{c}^{2}$, along with the differential energy spectrum for pp (blue) and ${ }^{7} \mathrm{Be}$ (cyan) neutrinos, and the electron recoil spectrum from the double beta decay of ${ }^{136} \mathrm{Xe}$ (green). Assumptions are $99.5 \%$ discrimination of electronic recoils, $50 \%$ acceptance of nuclear recoils, and $80 \%$ flat analysis cuts acceptance. Taken from [185].

about $100 \mathrm{~m}$. Inside the detector is foreseen an internal part (with a diameter of about $26 \mathrm{~m}$ ) containing about 50 kilotons of liquid scintillator, separated from a nonscintillating buffer region by a nylon barrier. Outside, a tank (made in steel or concrete) separates the inner detector from an outer water tank; it is used both for shielding and as an active muon veto. To collect the scintillation light, about 45,000 photomultipliers (with a diameter of $20 \mathrm{~cm}$ ) are mounted to the internal walls of the detector. To increase the optically active area, the photomultipliers tubes are equipped with conic mirrors, the corresponding surface coverage is about $30 \%$. Figure 22 shows a schematic overview of the current LENA design.

Among the favored solvent for the liquid scintillator in LENA, the LAB (linear alkylbenzene) is currently the preferred one. It has a high light yield and large attenuation length and it has also the advantage of being a nonhazardous liquid. The attenuation lengths is on the order of 10 to $20 \mathrm{~m}$ (at a wavelength of $430 \mathrm{~nm}$ ) and the photoelectron yield could be greater than 200 photoelectrons per $\mathrm{MeV}$ (with a scintillator mixture containing $2 \mathrm{~g} / \mathrm{L} \mathrm{PPO}$ and $20 \mathrm{mg} / \mathrm{L}$ bisMSB as wavelength shifters). Studies have been carried out to test the large-scale light transport and the differences in scintillator response for $\alpha, \beta$, and $\gamma$ particles. An alternative solvent option is the well-studied PXE [187] or a mixture of PXE and dodecane.

As already pointed out, Borexino has splendidly demonstrated the potential of the detection technique with liquid scintillator based detectors for the solar neutrino detection. This technique offers the opportunity for a spectrally resolved measurement of the solar neutrino spectrum in the all energy range. 


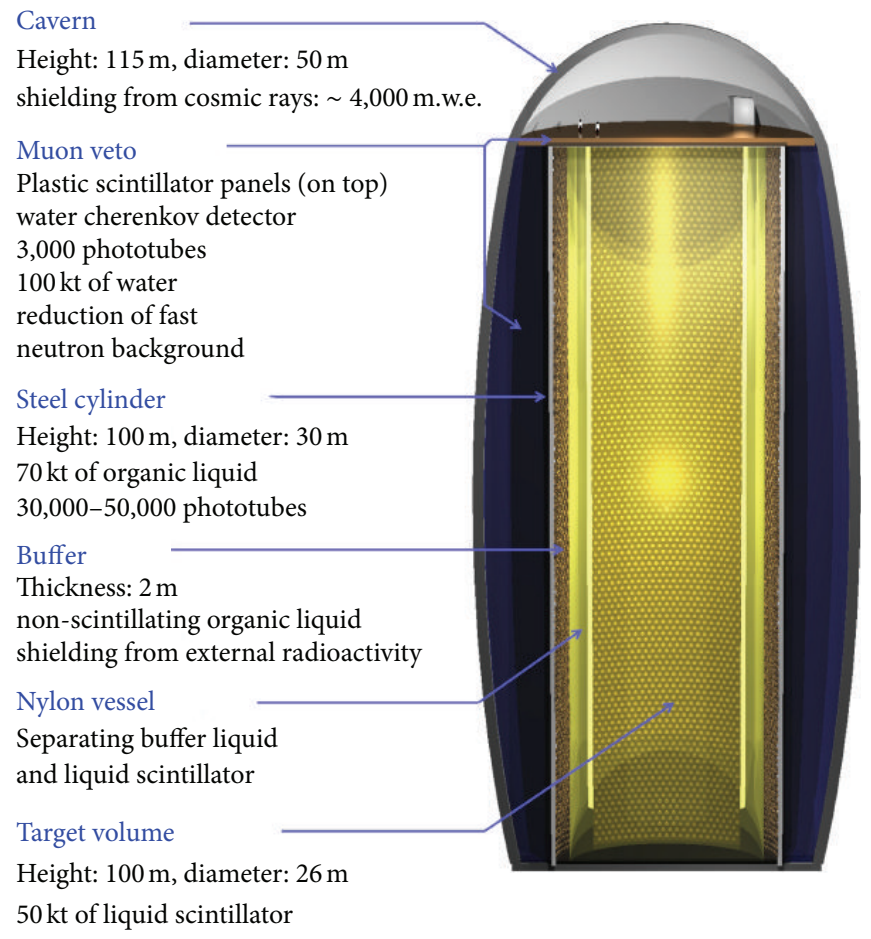

FIGURE 22: Schematical view of the LENA detector. From [186].

TABLE 9: Expected solar neutrino rates in LENA (channel $v e \rightarrow e v$ ). The estimates are derived from the existing Borexino analyses [154, 188] as well as expectation values for the respective energy windows (EW) of observation [189-191]. The quoted fiducial masses, $m_{\mathrm{fid}}$, in LAB are based on a Monte Carlo simulation of the external $\gamma$-ray background in LENA. Table taken and adapted from [186].

\begin{tabular}{lccc}
\hline Source & EW $[\mathrm{MeV}]$ & $m_{\text {fid }}[\mathrm{kt}]$ & Rate $[\mathrm{cpd}]$ \\
\hline$p p$ & $>0.25$ & 30 & 40 \\
$p e p$ & $0.8-1.4$ & 30 & $2.8 \times 10^{2}$ \\
${ }^{7 \hat{i}_{i} \hat{L}} \mathrm{Be}$ & $>0.25$ & 35 & $1.0 \times 10^{4}$ \\
${ }^{8 \hat{i}_{i} \hat{L}} \mathrm{~B}$ & $>2.8$ & 35 & 79 \\
$\mathrm{CNO}$ & $0.8-1.4$ & 30 & $1.9 \times 10^{2}$ \\
\hline
\end{tabular}

Because the smaller ratio of surface to volume compared to the Borexino detector in LENA it is very likely to reach the excellent background conditions of Borexino (A smaller ratio of surface to volume decreases the chance that the scintillator is contaminated with radioimpurities.) Monte Carlo simulations of the gamma background due to the uranium, thorium, and potassium from the photomultipliers glass show that a fiducial volume of the order of $30 \mathrm{ktons}$ is achievable for solar neutrino studies; LENA will be able to address topics both in neutrino oscillations and in solar physics thanks to its unprecedented statistics. A high statistics can be obtained in short times and in both Pyhsalmi and Frejus underground laboratories, where the detector could be hosted and where the cosmogenic background of ${ }^{11} \mathrm{C}$ will be significantly lower than in Borexino.

Monte Carlo simulations show that for pep, CNO, and low-energy ${ }^{8} \mathrm{~B}-\nu$ s detection a fiducial mass of $\sim 30 \mathrm{kton}$ is necessary, while the fiducial mass for ${ }^{7} \mathrm{Be}-v \mathrm{~s}$ and high-energy $(E>5 \mathrm{MeV}){ }^{8} \mathrm{~B}-v$ s could be enlarged to $35 \mathrm{kton}$ or more.

In Table 9 the expected rates in 30 kton for the neutrinos emitted in the $p p$ chain and the CNO-bicycle are reported, using the most recent solar model predictions. This evaluation refers to a detection threshold set at about $250 \mathrm{keV}$.

7.2.3. New Techniques with Organic Scintillators: LENS. The main goal of the Low Energy Neutrino Spectroscopy (LENS) detector is the real-time measurement of solar neutrinos as a function of their energy, focusing, on particular, in the analysis of the lowest energy neutrinos coming from the proton-proton fusion (i.e., the $p p$ neutrinos), which represent the main contribution and the less known component of the pp-chain of fusion reactions inside the Sun.

In order to make an energy spectrum measurement on low energy neutrinos, it is necessary to reach a low threshold for the charged current (CC) process and to be able to 
discriminate the background from radioactive decays. The CC process employed in LENS is the neutrino induced transition of ${ }^{115} \mathrm{In}$ to an excited state of ${ }^{115} \mathrm{Sn}$ :

$$
\begin{gathered}
\nu_{e}+{ }^{115} \mathrm{In} \longrightarrow{ }^{115} \mathrm{Sn}^{*}+e^{-} \quad\left(E=E_{\nu}-114 \mathrm{keV}\right) \\
{ }^{115} \mathrm{Sn}^{*}(\tau=4.76 \mu \mathrm{s}) \longrightarrow{ }^{115} \mathrm{Sn}+\gamma(498 \mathrm{keV})+\gamma(116 \mathrm{keV}) .
\end{gathered}
$$

Thanks to that it is possible to detect low energy neutrinos with a threshold of $114 \mathrm{keV}$ and measure their energy, following an idea that has been investigated since the 1970 s [192].

The primary interaction and the secondary cascade enable a triple coincidence, correlated in space and time. LENS employs as detection medium a liquid scintillator chemically doped with natural indium $\left({ }^{115} \mathrm{In}=95.7 \%\right)$. In order to exploit the spatial correlation, the volume of the detector is segmented into cubic cells $(7.5 \mathrm{~cm})$ by clear foils (Teflon FEP) that have a lower index of refraction than the liquid scintillator. By internal reflection, the scintillation light produced in a cell is channeled in the directions of the 6-cell faces. The collected channeled light is read out at the edge of the detector by photomultiplier tubes.

LENS should be able to determine the low energy solar neutrino fluxes with an accuracy $\leq 4 \%$, testing neutrino and solar physics with a global precision better than the present one and also looking for any inconsistency in the LMA conversion mechanism [193].

\section{Remarks on the Present Status and Future Outlooks}

In this paper we went through the main results obtained in the study of solar neutrinos and we focused our attention mainly on the last decade advancements. Since the discovery of neutrino oscillations and the solution to the long standing solar neutrino problem at the dawn of the century, solar neutrino physics has evolved quickly and has become a mature field. It took advantage from the great amount of data of the different working phases of experiments like Super-Kamiokande and $\mathrm{SNO}$, corroborated by the analogous studies of the reactor experiment KamLAND and recently sustained also by the first "low energy" real-time measurements performed by Borexino.

A coherent picture emerges from all of these experimental achievements and from the global analyses performed by the single experimental collaborations and in other parallel theoretical and phenomenological studies and we can say that the basic properties and the values of the parameters driving solar neutrino oscillations, $\Delta m_{12}^{2}$, and $\theta_{12}$ are now known with quite a satisfactory accuracy (Tables 7 and 8) and the validity of the MSW-LMA solution is well established (Figure 18). Nevertheless, there are still important aspects of the oscillation mechanism on which we would like to shed more light. In particular the vacuum to matter transition regime requires further study (see also Section 9).
Important parallel advancements took place in the study of neutrino oscillations during the very last years; in particular a series of reactor short baseline experiments have confidently established that the mixing angle $\theta_{13}$ is different from zero, opening interesting opportunities for future experiments looking for leptonic CP violation (the details have been given in Section 6).

From an astrophysical point of view, current solar neutrino data have put us on the verge of fulfilling the original dream of Davies and Bahcall and use solar neutrinos as a probe of the nuclear fusion processes that power the Sun and the vast majority of stars. The four relevant neutrino fluxes produced in the different pp-chains are, thanks to the latest efforts from Borexino, now very well constrained from experimental data $\left({ }^{7}\right.$ Be has the largest uncertainty, $4.5 \%$, if the solar luminosity constraint is used in the analysis of the data). Standard solar model predictions are in excellent agreement with solar fluxes, regardless of the solar composition assumed in the construction of the model. In fact, the agreement is so good for both high- $Z$ and low-Z solar models (Table 4) that it is very unlikely that neutrino fluxes from pp-chains will be able to discriminate between solar compositions. Borexino has also recently established the most stringent upper limit on CNO fluxes which is now only a factor of 1.5 larger than solar model predictions. CNO fluxes carry a wealth of information about the solar core and are, for solar physics, the most coveted prize. The latest developments, both experimentally and in background subtraction techniques, place Borexino at the doors of this momentous measurement. In this regard, potentiality of $\mathrm{SNO}+$ is unmatched, but at present the primary solar neutrino measurements are planned to take place after the double beta decay measurements are carried out for at least 4 years. Some of the possibilities that a measurement of CNO fluxes would offer are discussed in the next section, together with some of the main questions which are still open.

\section{Open Questions in Solar Neutrino Physics}

9.1. The Metallicity Problem. The solar abundance or solar metallicity problem has been around for some time now. In analogy with the solar neutrino problem, there have been attempts (although in most cases, it is fair to say, of somewhat less radical nature) to solve it by introducing modifications to the input physics of SSMs. To mention a few of them, we could remember the following:

(i) large enhancement of $\mathrm{Ne}$ abundance $[194,195]$ is important because of its contribution uncertainty and a weak bond in solar abundances because its abundance is determined rather indirectly [44];

(ii) increased element diffusion rates $[67,196]$;

(iii) accretion of metal-poor materials leading to a "twozone" solar model in terms of composition [64, 196, 197].

Also solar models including some sort of prescriptions to account for rotation and other dynamical effects have been put forward; however, their performance is quite poor. 
So far, all attempts of finding a solution to all the manifestations of the solar abundance problem have failed. In some cases, $Y_{S}$ can be brought into agreement with helioseismology, in other cases $R_{\mathrm{CZ}}$ and the sound speed profile, but a simultaneous solution to all the problems has not yet been found.

The exceptions are the two obvious ones: (a) the low$Z$ solar abundances actually underestimate the true metal content of the Sun; (b) an increase of radiative opacities by the right amount (15\% to $20 \%$ at the base of the convective zone down to about $3 \%$ in the solar core) to compensate for the decrease induced by the low- $Z$ abundances. The drawback to this idea is that current state-of-the-art radiative opacity calculations differ by only 2 to $3 \%$ at the base of the convective envelope, much lower from what would be required by low $-Z$ models.

It has indeed been shown that by increasing the radiative opacity in low-Z SSMs the agreement with helioseismology can be restored to match results from high-Z SSMs [198]. Additionally, the ${ }^{7} \mathrm{Be}$ and ${ }^{8} \mathrm{~B}$ fluxes of a low-Z SSMs with increased opacity coincide with those from a high- $Z$ model [199]. As good as this may seem, it shows the intrinsic degeneracy between composition and opacities.

Recently, a novel approach, the linear solar models (LSM), that relate changes in solar observables to modifications in the input physics by the calculation of kernels based on SSMs has been developed by [200] (We remark that LSMs offer an efficient way of studying the response of the solar structure to changes in any of the physical ingredients entering solar model calculations that does not require the construction of solar models with the varied physics.). LSMs have been applied in particular to the solar abundance problem and the changes required in the radiative opacity to restore the agreement between low- $Z$ models and helioseismology [201] quantitatively, result similar to those quoted above.

By using ${ }^{8} \mathrm{~B}$ and now ${ }^{7} \mathrm{Be}$ as thermometers of the solar core [79], CNO neutrinos represent a unique way to break this degeneracy and provide an independent determination of the $\mathrm{CNO}$ abundances, particularly the $\mathrm{C}+\mathrm{N}$ abundance in the solar core. Keeping in mind the antagonism between solar interior and solar atmosphere models that the solar abundance problem has established, results from $\mathrm{CNO}$ fluxes will be of the outmost relevance for solar, and by extension stellar, physics.

9.2. The Vacuum to Matter Transition? Solar neutrino experiments already measured the two-extreme-flavor conversion regimes, the vacuum term domination and matter term domination. There is no direct experimental evidence of the transition from one to the other. In fact, the lower energetic ${ }^{8} \mathrm{~B}$ neutrinos are sensitive to the rise of the spectrum from matter combination towards vacuum, but the data (still very uncertain) seem not to show it. More data coming from SuperKamiokande, Borexino, and $\mathrm{SNO}+$ experiments will further explore the conversion in this regime.

The precise measurement of low energy neutrinos like pep, exploiting the fact that are more energetic than ${ }^{7} \mathrm{Be}$ neutrinos, will also help to see small solar matter effects in the flavor conversion. This matter effects will be more precisely determined by the comparison of pep and pp neutrino measurements. In fact, the low energy neutrinos that are better suited to test matter effects are the CNO neutrinos. While the CNO neutrinos energy is around the pep neutrinos energy, the former are produced at higher temperatures and therefore at higher densities. The larger matter density where neutrinos are produced leads to larger matter effects for CNO neutrinos than for pep neutrinos. In fact, matter effects produce a significant spectral tilt of CNO neutrinos ( $10 \%)$, which might be a good handle to separate the signal for background.

The determination of the vacuum to matter transition has a significant impact on the determination of the solar mass splitting derived by solar data, which adds to the implications of Earth-matter effects measured by comparing the neutrino fluxes during the day and night. The good match of the independently determined solar mass splitting by solar neutrino experiments and by reactor experiments leads to the best test on nonstandard neutrino physics to solar neutrinos. There are many possibilities but the two scenarios more studied are the addition of new neutral current interactions $[168,202]$ which modify the amplitude of matter effects and therefore shift the effective mass splitting and the existence of a sterile neutrino which adds a new state with the appropriate mass splitting [169] to produce deviations of the flavor conversion in the 1$3 \mathrm{MeV}$ range.

9.3. What Else Can We Learn from CNO Fluxes? The most fundamental information the CNO fluxes carry is the most obvious one: that the CNO-bicycle operates in stars and it is a viable process for hydrogen fusion. It must not be forgotten that neutrinos are the only direct evidence of nuclear reactions being the source of energy in solar (stellar) interiors. For the Sun, models predict a marginal contribution to the total energy budget from CNO reactions, $0.8 \%$ and $0.4 \%$ for high $-Z$ and low $Z$ solar models. However, CNO becomes the dominant mode for hydrogen burning in stars with masses right above the solar value. Detection of CNO neutrinos will provide direct evidence that $\mathrm{CNO}$ reactions actually take place in nature, as originally envisioned by Bethe [203]; it has been a long wait.

The second important aspect of $\mathrm{CNO}$ neutrinos is the information they provide about the abundance of metals in the solar core. Knowing the abundance of CNO elements in the solar core is important by itself. In particular, a "perfect" measurement of the combined ${ }^{13} \mathrm{~N}+{ }^{15} \mathrm{O}$ flux translates into a determination of the solar $\mathrm{C}+\mathrm{N}$ abundance with $\sim 10 \%$ uncertainty [79], and the dominant sources of uncertainties are experimental and can be potentially reduced. Assuming we know the solar surface abundance of the same elements, that is, let us forget for the time being about the solar abundance problem, we can then put constraints on mixing mechanisms that may have created composition gradients during the evolution of the Sun. SSMs predict that the number density of $\mathrm{C}+\mathrm{N}$ is enhanced in the solar core, at present day, by $\sim 16 \%$ with respect to the surface due to the effects of the microscopic diffusion. And, although helioseismology shows that models with diffusion work much better than models 
without, there is no direct evidence of how efficient diffusion is. In fact, there have been suggestions that the standard prescription [204] may be too efficient in the Sun [205] and that diffusion rates should be lowered by $\sim 15 \%$. Solar CNO neutrinos could provide a test for the efficiency of the diffusion.

There are other possibilities that might create a contrast between the solar core and surface composition. Recently, it has been shown that the Sun has a peculiar composition when compared to "solar twins," that is, stars almost identical to the Sun in their surface properties [206, 207]. The authors found that the Sun is enhanced in volatile elements with respect to the solar twins that show no sign of harbouring planets by about $20 \%$. In fact, they have associated this fact to the presence of rocky planets in the solar system, where refractory elements are locked, and the occurrence of an accretion episode of volatile-enriched material [207] after rocky cores is formed in the protoplanetary disk. If this was true, then the Sun would have an envelope that is richer in CNO than its interior. If a measurement of the ${ }^{13} \mathrm{~N}+{ }^{15} \mathrm{O}$ flux would yield as a result a core composition where the abundance of $\mathrm{C}+\mathrm{N}$ would be comparable or less than the surface value, then we would have an extremely exciting piece of evidence about the earlier phases of planet formation in the solar system [79].

\section{Acknowledgments}

C. Pena-Garay is supported in part by the Spanish MICINN Grants FPA-2007-60323 and FPA2011-29678, the Generalitat Valenciana Grant PROMETEO/2009/116, and the ITN INVISIBLES (Marie Curie Actions, PITN-GA-2011-289442). A. Serenelli is supported by the European Union International Reintegration Grant PIRG-GA-2009-247732 and the MICINN Grant AYA2011-24704. The authors are all grateful to the editors of this special issue on neutrino physics for their invitation and support.

\section{References}

[1] S. M. Bilenky, C. Giunti, and W. Grimus, "Phenomenology of neutrino oscillations," Progress in Particle and Nuclear Physics, vol. 43, no. 1, pp. 1-86, 1999.

[2] G. Altarelli and F. Feruglio, "Models of neutrino masses and mixings," New Journal of Physics, vol. 6, pp. 1-39, 2004.

[3] A. Strumia and F. Vissani, "Implications of neutrino data circa 2005,” Nuclear Physics B, vol. 726, no. 1-2, pp. 294-316, 2005.

[4] A. Strumia and F. Vissani, "Neutrino masses and mixings and...," http://arxiv.org/abs/hep-ph/0606054.

[5] R. N. Mohapatra, S. Antusch, K. S. Babu et al., "Theory of neutrinos," submitted, http://arxiv.org/abs/hep-ph/0412099.

[6] T. Hambye, Y. Lin, A. Notari, M. Papucci, and A. Strumia, "Constraints on neutrino masses from leptogenesis models," Nuclear Physics B, vol. 695, no. 1-2, pp. 169-191, 2004.

[7] R. N. Mohapatra and A. Y. Smirnov, "Neutrino mass and new physics," Annual Review of Nuclear and Particle Science, vol. 56, pp. 569-628, 2006.

[8] R. Barbieri, P. Creminelli, and A. Strumia, "Neutrino oscillations and large extra dimensions," Nuclear Physics B, vol. 585, no. 1-2, pp. 28-44, 2000.
[9] N. Arkani-Hamed, S. Dimopoulos, G. R. Dvali, and J. MarchRussell, "Neutrino masses from large extra dimensions," Physical Review D, vol. 65, no. 2, Article ID 024032, 2002.

[10] G. Altarelli and F. Feruglio, "Tri-bimaximal neutrino mixing from discrete symmetry in extra dimensions," Nuclear Physics B, vol. 720, no. 1-2, pp. 64-88, 2005.

[11] G. C. Branco, R. González Felipe, F. R. Joaquim, and M. N. Rebelo, "Leptogenesis, CP violation and neutrino data: What can we learn?" Nuclear Physics B, vol. 640, no. 1-2, pp. 202-232, 2002.

[12] E. K. Akhmedov, M. Frigerio, and A. Y. Smirnov, "Probing the seesaw mechanism with neutrino data and leptogenesis," Journal of High Energy Physics, vol. 309, p. 21, 2003.

[13] T. Hambye, Y. Lin, A. Notari, M. Papucci, and A. Strumia, "Constraints on neutrino masses from leptogenesis models," Nuclear Physics B, vol. 695, no. 1-2, pp. 169-191, 2004.

[14] J. N. Bahcall, "Solar neutrinos. I. Theoretical," Physical Review Letters, vol. 12, pp. 300-302, 1964.

[15] R. J. Davis, "Solar neutrinos. II. Experimental," Physical Review Letters, vol. 12, pp. 302-305, 1964.

[16] R. J. Davis, D. S. Harmer, and K. C. Hoffman, "Search for neutrinos from the sun," Physical Review Letters, vol. 20, pp. 12051209, 1968.

[17] L. Miramonti and F. Reseghetti, "Solar neutrino physics: historical evolution, present status and perspectives," Rivista del Nuovo Cimento, vol. 25, no. 7, pp. 1-128, 2002.

[18] B. Pontecorvo, "Mesonium and anti-mesonium," Journal of Experimental and Theoretical Physics, vol. 33, p. 549, 1957, Soviet Physics, vol. 6, pp. 429, 1958.

[19] B. Pontecorvo, "Inverse beta processes and nonconservation of lepton charge," Journal of Experimental and Theoretical Physics, vol. 34, p. 247, 1958, Soviet Physics, vol. 7, pp. 172, 1958.

[20] K. S. Hirata, T. Kajita, T. Kifune et al., "Observation of ${ }^{8}$ B solar neutrinos in the Kamiokande-II detector," Physical Review Letters, vol. 63, pp. 16-19, 1989.

[21] Y. Fukuda, T. Hayakawa, E. Ichihara et al., "Measurement of the solar neutrino energy spectrum using neutrino-electron scattering," Physical Review Letters, vol. 82, pp. 2430-2434, 1999.

[22] W. Hampel, J. Handta, G. Heusser et al., "GALLEX solar neutrino observations: results for GALLEX IV," Physics Letters B, vol. 447, pp. 127-133, 1999.

[23] M. Altmann, M. Balatab, P. Bellic et al., "Complete results for five years of GNO solar neutrino observations," Physics Letters $B$, vol. 616, pp. 174-190, 2005.

[24] J. N. Abdurashitov, T. J. Bowles, M. L. Cherry et al., "Measurement of the solar neutrino capture rate by SAGE and implications for neutrino oscillations in vacuum," Physical Review Letters, vol. 83, pp. 4686-4689, 1999.

[25] Q. R. Ahmad, R. C. Allen, T. C. Andersen et al., "Measurement of the rate of $v_{e}+d \rightarrow p+p+e^{-}$interactions produced by ${ }^{8} \mathrm{~B}$ solar neutrinos at the sudbury neutrino observatory," Physical Review Letters, vol. 87, Article ID 071301, 2001.

[26] S. Fukuda, Y. Fukuda, M. Ishitsuka et al., "Solar ${ }^{8}$ B and hep neutrino measurements from 1258 days of super-kamiokande data," Physical Review Letters, vol. 86, pp. 5651-5655, 2001.

[27] J. N. Bahcall, M. C. Gonzalez-Garcia, and C. Peña-Garay, "Global analysis of solar neutrino oscillations including SNO CC measurement," Journal of High Energy Physics, vol. 8, p. 14, 2001.

[28] G. L. Fogli, E. Lisi, D. Montanino, and A. Palazzo, "Modeldependent and independent implications of the first Sudbury 
Neutrino Observatory results," Physical Review D, vol. 64, Article ID 093007, 2001.

[29] Q. R. Ahmad, R. C. Allen, T. C. Andersen et al., "Direct evidence for neutrino flavor transformation from neutral-current interactions in the Sudbury Neutrino Observatory," Physical Review Letters, vol. 89, no. 1, Article ID 011301, 6 pages, 2002.

[30] S. N. Ahmed, A. E. Anthony, E. W. Beier et al., "Measurement of the total active ${ }^{8} \mathrm{~B}$ solar neutrino flux at the sudbury neutrino observatory with enhanced neutral current sensitivity," Physical Review Letters, vol. 92, no. 18, Article ID 181301, 6 pages, 2004.

[31] B. Aharmim, S. N. Ahmed, J. F. Amsbaugh et al., "Independent measurement of the total active ${ }^{8} \mathrm{~B}$ solar neutrino flux using an array of ${ }^{3} \mathrm{He}$ proportional counters at the sudbury neutrino observatory," Physical Review Letters, vol. 101, Article ID 111301, 5 pages, 2008.

[32] Q. R. Ahmad, R. C. Allen, T. C. Andersen et al., "Measurement of day and night neutrino energy spectra at SNO and constraints on neutrino mixing parameters," Physical Review Letters, vol. 89, no. 1, Article ID 011302, 5 pages, 2002.

[33] R. Davis, "Memories of a Nobel laureate," CERN Courier, vol. 42, no. 10, p. 15, 2002.

[34] K. Eguchi, S. Enomoto, K. Furuno et al., "First results from KamLAND: evidence for reactor antineutrino disappearance," Physical Review Letters, vol. 90, Article ID 021802, 6 pages, 2003.

[35] J. N. Bahcall, M. Pinsonneault, and S. Basu, "Solar models: current epoch and time dependences, neutrinos, and helioseismological properties," The Astrophysical Journal, vol. 555, no. 2, p. 990, 2001.

[36] A. Piepke, "KamLAND: a reactor neutrino experiment testing the solar neutrino anomaly," Nuclear Physics B, vol. 91, pp. 99104, 2001.

[37] A. de Gouvea and C. Peña-Garay, "Solving the solar neutrino puzzle with KamLAND and solar data," Physical Review D, vol. 64, Article ID 0113011, 8 pages, 2001.

[38] P. Aliani, V. Antonelli, M. Picariello, and E. Torrente-Lujan, "KamLAND and the determination of neutrino mixing parameters in the post SNO-NC era," New Journal of Physics, vol. 5, p. 2, 2003.

[39] T. Araki, K. Eguchi, S. Enomoto et al., "Measurement of neutrino oscillation with KamLAND: evidence of spectral distortion," Physical Review Letters, vol. 94, Article ID 081801, 8 pages, 2005.

[40] S. Abe, T. Ebihara, S. Enomoto et al., "Precision measurement of neutrino oscillation parameters with KamLAND," Physical Review Letters, vol. 100, no. 22, Article ID 221803, 5 pages, 2008.

[41] A. Gando, Y. Gando, K. Ichimura et al., "Constraints on $\theta_{13}$ from a three-flavor oscillation analysis of reactor antineutrinos at KamLAND," Physical Review D, vol. 83, Article ID 052002, 11 pages, 2011.

[42] J. N. Bahcall, W. F. Huebner, S. H. Lubow, P. D. Parker, and R. K. Ulrich, "Standard solar models and the uncertainties in predicted capture rates of solar neutrinos," Reviews of Modern Physics, vol. 54, pp. 767-799, 1982.

[43] J. N. Bahcall, Neutrino Astrophysics, Cambridge University Press, 1989.

[44] K. Lodders, H. Palme, and H.-P. Gail, "Abundances of the elements in the solar system," submitted, http://arxiv.org/abs/ 0901.1149.

[45] B. Beeck, R. Collet, M. Steffen et al., "Simulations of the solar near-surface layers with the CO5BOLD, MURaM, and Stagger codes," Astronom \& Astrophys, vol. 539, article 121, 11 pages, 2012.
[46] M. Asplund, N. Grevesse, A. J. Sauval, and P. Scott, "The chemical composition of the sun," Annual Review of Astronomy and Astrophysics, vol. 47, pp. 481-522, 2009.

[47] M. Asplund, N. Grevesse, and J. Sauval, "The solar chemical composition," in Cosmic Abundances as Records of Stellar Evolution and Nucleosynthesis, T. G. Barnes III and F. N. Bash, Eds., vol. 336, p. 25, 2005.

[48] C. Allende Prieto, D. L. Lambert, and M. Asplund, “The forbidden abundance of oxygen in the sun," The Astrophysical Journal Letters, vol. 556, p. L63, 2001.

[49] N. Grevesse and A. Noels, "Solar composition," in Origin and Evolution of Elements, N. Prantzos, E. Vangioni-Flam, and M. Casse, Eds., p. 15, 1993.

[50] N. Grevesse and A. J. Sauval, "Standard solar composition," Space Science Reviews, vol. 85, no. 1-2, pp. 161-174, 1998.

[51] E. Caffau, H. G. Ludwig, M. Steffen, B. Freytag, and P. Bonifacio, "Solar chemical abundances determined with a CO5BOLD 3D model atmosphere," Solar Physics, vol. 268, no. 2, pp. 255-269, 2011.

[52] C. A. Iglesias and F. J. Rogers, "Updated opal opacities," The Astrophysical Journal, vol. 464, p. 943, 1996.

[53] N. R. Badnell, M. A. Bautista, K. Butler et al., "Updated opacities from the opacity project," Monthly Notices of the Royal Astronomical Society, vol. 360, no. 2, pp. 458-464, 2005.

[54] J. W. Ferguson, D. R. Alexander, F. Allard et al., "Low-temperature opacities," The Astrophysical Journal, vol. 623, p. 585, 2005.

[55] E. G. Adelberger, A. García, R. G. H. Robertson et al., "Solar fusion cross sections. II. The pp chain and CNO cycles," Reviews of Modern Physics, vol. 83, pp. 195-245, 2011.

[56] E. G. Adelberger, S. M. Austin, J. N. Bahcall et al., "Solar fusion cross sections," Reviews of Modern Physics, vol. 70, pp. 12651291, 1998.

[57] J. Christensen Dalsgaard, "Helioseismology," Reviews of Modern Physics, vol. 74, pp. 1073-1129, 2002.

[58] J. Christensen Dalsgaard, W. Dappen, S. V. Ajukov et al., "The current state of solar modeling," Science, vol. 272, pp. 1286-1292, 1996.

[59] S. Basu and H. M. Antia, "Seismic measurement of the depth of the solar convection zone," Monthly Notices of the Royal Astronomical Society, vol. 287, no. 1, pp. 189-198, 1997.

[60] S. Basu and H. Antia, "Constraining solar abundances using helioseismology," The Astrophysical Journal Letters, vol. 606, no. 1, p. 85L, 2004.

[61] S. Basu et al. "Solar internal sound speed as inferred from combined BiSON and LOWL oscillation frequencies," Monthly Notices of the Royal Astronomical Society, vol. 292, no. 2, pp. 243251, 1997.

[62] A. G. Kosovichev, J. Schou, P. H. Scherrer et al., "Structure and rotation of the solar interior: initial results from the Mdi medium-L program," Solar Physics, vol. 170, no. 1, pp. 43-61, 1997.

[63] S. Basu, W. J. Chaplin, Y. Elsworth, R. New, and A. M. Serenelli, "Fresh insights on the structure of the solar core," Astrophysical Journal Letters, vol. 699, no. 2, pp. 1403-1417, 2009.

[64] A. M. Serenelli, W. C. Haxton, and C. Peña-Garay, "Solar models with accretion. I. Application to the solar abundance problem," The Astrophysical Journal, vol. 743, p. 24, 2011.

[65] H. M. Antia and S. Basu, "Determining solar abundances using helio seismology," Astrophysical Journal Letters, vol. 644, no. 2, pp. 1292-1298, 2006. 
[66] W. J. Chaplin, A. M. Serenelli, S. Basu, Y. Elsworth, R. New, and G. A. Verner, "Solar heavy-element abundance: constraints from frequency separationjiatios of low-degree p-modes," Astrophysical Journal Letters, vol. 670, no. 1, pp. 872-884, 2007.

[67] J. Montalban, A. Miglio, A. Noels, and N. Grevesse, "In SOHO 14 Helio- and asteroseismology: towards a golden future," ESA Special Publication, vol. 559, p. 574, 2004.

[68] S. Turck-Chièze, S. Couvidat, L. Piau et al., "Surprising sun: a new step towards a complete picture?" Physical Review Letters, vol. 93, Article ID 211102, 4 pages, 2004.

[69] J. N. Bahcall, S. Basu, M. H. Pinsonneault, and A. M. Serenelli, "Helioseismological implications of recent solar abundance determinations," Astrophysical Journal, vol. 618, no. 2, pp. 10491056, 2005.

[70] F. Delahaye and M. H. Pinsonneault, "The solar heavy-element abundances. I. Constraints from stellar interiors," Astrophysical Journal, vol. 649, no. 1, pp. 529-540, 2006.

[71] A. M. Serenelli and S. Basu, "Determining the initial helium abundance of the sun," Astrophysical Journal Letters, vol. 719, no. 1, pp. 865-872, 2010.

[72] A. M. Serenelli, S. Basu, J. W. Ferguson, and M. Asplund, "New solar composition: the problem with solar models revisited," Astrophysical Journal Letters, vol. 705, no. 2, pp. L123-L127, 2009.

[73] I. W. Roxburgh and S. V. Vorontsov, "The ratio of small to large separations of acoustic oscillations as a diagnostic of the interior of solar-like stars," Astronomy \& Astrophysics, vol. 411, pp. 215220, 2003.

[74] G. Bellini, J. Benziger, D. Bick et al., "First evidence of pep solar neutrinos by direct detection in borexino," Physical Review Letters, vol. 108, Article ID 051302, 6 pages, 2012.

[75] D. D. Clayton, Principles of Stellar Evolution and Nucleosynthesis, University of Chicago Press, 1984.

[76] J. N. Bahcall and M. H. Pinsonneault, "What do we (not) know theoretically about solar neutrino fluxes?" Physical Review Letters, vol. 92, no. 12, Article ID 121301, 2004.

[77] A. Formicola, G. Imbrianib, H. Costantini et al., "Astrophysical $S$-factor of ${ }^{14} \mathrm{~N}(\mathrm{p}, \gamma){ }^{15} \mathrm{O}$," Physics Letters B, vol. 591, pp. 61-68, 2004.

[78] M. Marta, A. Formicola, Gy. Gyürky et al., "Precision study of ground state capture in the ${ }^{14} \mathrm{~N}(\mathrm{p}, \gamma){ }^{15} \mathrm{O}$ reaction," Physical Review C, vol. 78, Article ID 022802, 4 pages, 2008.

[79] W. C. Haxton and A. M. Serenelli, "CN cycle solar neutrinos and the Sun's primordial core metallicity," Astrophysical Journal Letters, vol. 687, no. 1, pp. 678-691, 2008.

[80] J. N. Bahcall, A. M. Serenelli, and S. Basu, "10,000 Standard solar models: a monte carlo simulation," Astrophysical Journal, Supplement Series, vol. 165, no. 1, pp. 400-431, 2006.

[81] J. N. Bahcall and A. M. Serenelli, "How do uncertainties in the surface chemical composition of the sun affect the predicted solar neutrino fluxes?” Astrophysical Journal, vol. 626, no. 1, pp. 530-542, 2005.

[82] P. C. de Holanda and A. Y. Smirnov, "Solar neutrinos: global analysis with day and night spectra from SNO," Physical Review $D$, vol. 66, Article ID 113005, 10 pages, 2002.

[83] P. C. de Holanda and A. Y. Smirnov, "LMA MSW solution of the solar neutrino problem and first KamLAND results," Journal of Cosmology and Astroparticle Physics, vol. 2003, no. 2, p. 1, 2003.

[84] J. N. Bahcall, M. C. Gonzalez-Garcia, and C. Peña-Garay, "Before and after: how has the SNO neutral current measurement changed things?" Journal of High Energy Physics, vol. 207, p. $54,2002$.
[85] J. N. Bahcall, M. C. Gonzalez-Garcia, and C. Peña-Garay, "Solar neutrinos before and after KamLAND," Journal of High Energy Physics, vol. 302, p. 9, 2003.

[86] G. L. Fogli, E. Lisi, A. Marrone, D. Montanino, A. Palazzo, and A. M. Rotunno, "Solar neutrino oscillation parameters after first KamLAND results," Physical Review D, vol. 67, no. 7, Article ID 073002, 2003.

[87] S. Pascoli and S. T. Petcov, "The SNO solar neutrino data, neutrinoless double beta-decay and neutrino mass spectrum," Physics Letters B, vol. 544, pp. 239-250, 2002.

[88] M. Maltoni, T. Schwetz, and J. W. F. Valle, "Combining first KamLAND results with solar neutrino data," Physical Review D, vol. 67, Article ID 093003, 2003.

[89] P. Aliani, V. Antonelli, M. Picariello, and E. Torrente-Lujan, "The Neutrino mass matrix after Kamland and SNO salt enhanced results," http://arxiv.org/abs/hep-ph/0309156. In press.

[90] P. Aliani, V. Antonelli, M. Picariello, and E. Torrente-Lujan, "Neutrino mass parameters from Kamland, SNO, and other solar evidence," Physical Review D, vol. 69, no. 1, Article ID 013005, 7 pages, 2004.

[91] A. Bandyopadhyay, S. Choubey, S. Goswami, and D. P. Roy, "Implications of the first neutral current data from SNO for solar neutrino oscillation," Physics Letters B, vol. 540, no. 1-2, pp. 14-19, 2002.

[92] V. Barger, D. Marfatia, K. Whisnant, and B. P. Wood, "Imprint of SNO neutral current data on the solar neutrino problem," Physics Letters B, vol. 537, no. 3-4, pp. 179-186, 2002.

[93] S. M. Bilenky, C. Giunti, J. A. Grifols, and E. Massó, "Absolute values of neutrino masses: status and prospects," Physics Reports, vol. 379, no. 2, pp. 69-148, 2003.

[94] B. Aharmim, S. N. Ahmed, A. E. Anthony et al., "Electron energy spectra, fluxes, and day-night asymmetries of ${ }^{8} \mathrm{~B}$ solar neutrinos from measurements with $\mathrm{NaCl}$ dissolved in the heavy-water detector at the Sudbury Neutrino Observatory," Physical Review C, vol. 72, Article ID 055502, 47 pages, 2005.

[95] M. B. Smy, Y. Ashie, S. Fukuda et al., "Precise measurement of the solar neutrino day-night and seasonal variation in SuperKamiokande-I," Physical Review D, vol. 69, Article ID 011104, 5 pages, 2004.

[96] P. Langacker, S. T. Petcov, G. Steigman, and S. Toshev, "Implications of the mikheyev-smirnov-wolfenstein (MSW) mechanism of amplification of neutrino oscillations in matter," Nuclear Physics B, vol. 282, pp. 589-609, 1987.

[97] S. T. Petcov, "Exact analytic description of two-neutrino oscillations in matter with exponentially varying density," Physics Letters B, vol. 200, pp. 373-379, 1988.

[98] S. T. Petcov, "Describing analytically the matter-enhanced twoneutrino transitions in a medium," Physics Letters B, vol. 406, pp. 355-365, 1997.

[99] P. I. Krastev and S. T. Petcov, "Resonance amplification and T-violation effects in three-neutrino oscillations in the earth," Physics Letters B, vol. 205, pp. 84-92, 1988.

[100] P. I. Krastev and S. T. Petcov, "Testing the vacuum oscillation and the MSW solutions of the solar neutrino problem," Nuclear Physics B, vol. 449, pp. 605-627, 1995.

[101] M. Chizhov, M. Maris, and S. T. Petcov, "On the oscillation length resonance in the transitions of solar and atmospheric neutrinos crossing the earth core," submitted, http://arxiv.org/ abs/hep-ph/9810501.

[102] P. C. de Holanda, W. Liao, and A. Y. Smirnov, "Toward precision measurements in solar neutrinos," Nuclear Physics B, vol. 702, no. 1-2, pp. 307-332, 2004. 
[103] A. N. Ioannisian, N. A. Kazarian, A. Y. Smirnov, and D. Wyler, "Precise analytical description of the Earth matter effect on oscillations of low energy neutrinos," Physical Review D, vol. 71, no. 3, Article ID 033006, 8 pages, 2005.

[104] E. K. Akhmedov, M. A. Tortola, and J. W. F. Valle, "A simple analytic three-flavour description of the day-night effect in the solar neutrino flux," Journal of High Energy Physics, vol. 405, p. 57, 2004.

[105] A. Friedland, C. Lunardini, and C. Peña-Garay, "Solar neutrinos as probes of neutrino-matter interactions," Physics Letters B, vol. 594, pp. 347-354, 2004.

[106] W. Liao, "Precise formulation of neutrino oscillation in the earth," Physical Review D, vol. 77, Article ID 053002, 11 pages, 2008.

[107] P. Aliani, V. Antonelli, M. Picariello, and E. Torrente-Lujan, "Global analysis of Solar neutrino oscillation evidence including SNO and implications for Borexino," Nuclear Physics B, vol. 634, no. 1-2, pp. 393-409, 2002.

[108] E. Torrente-Lujan, "Finite dimensional systems with random external fields and neutrino propagation in fluctuating media," Physical Review D, vol. 59, Article ID 073001, 7 pages, 1999.

[109] D. Dooling, C. Giunti, K. Kang, and C. W. Kim, "Matter effects in four-neutrino mixing," Physical Review D, vol. 61, Article ID 073011, 18 pages, 2000.

[110] J. Hosaka, K. Ishihara, J. Kameda et al., "Solar neutrino measurements in Super-Kamiokande-I," Physical Review D, vol. 73, Article ID 112001, 33 pages, 2006.

[111] C. Arpesella, H. O. Back, M. Balata et al., "Direct measurement of the ${ }^{7}$ Be solar neutrino flux with 192 days of Borexino data," Physical Review Letters, vol. 101, Article ID 091302, 6 pages, 2008.

[112] B. Aharmim, S. N. Ahmed, A. E. Anthony et al., "Low-energythreshold analysis of the Phase I and Phase II data sets of the Sudbury Neutrino Observatory," Physical Review C, vol. 81, Article ID 055504, 49 pages, 2010.

[113] B. Aharmim, Q. R. Ahmad, S. N. Ahmed et al., "Determination of the $v_{e}$ and total ${ }^{8} \mathrm{~B}$ solar neutrino fluxes using the Sudbury Neutrino Observatory Phase I data set," Physical Review C, vol. 75, Article ID 045502, 69 pages, 2007.

[114] J. P. Cravens, K. Abe, T. Iida et al., "Solar neutrino measurements in Super-Kamiokande-II," Physical Review D, vol. 78, Article ID 032002, 11 pages, 2008.

[115] K. Abe, Y. Hayato, T. Iida et al., "Solar neutrino results in SuperKamiokande-III," Physical Review D, vol. 83, Article ID 052010, 19 pages, 2011.

[116] M. Smy, "Low energy neutrino astronomy in super-kamiokande," in Proceedings of the Meeting of the American Physical Society Division of Particles and Fields at Brown University, 2011, http://arxiv.org/abs/1110.0012.

[117] G. Alimonti, C. Arpesella, H. Backc et al., "The Borexino detector at the Laboratori Nazionali del Gran Sasso," Nuclear Instruments and Methods in Physics Research A, vol. 600, pp. 568-593, 2009.

[118] C. Peña-Garay, "talk at the conference Neutrino Telescopes 2007," March, Venice, Italy, 2007, http://neutrino.pd.infn.it/ conference2007/.

[119] C. Arpesella, G. Bellinib, J. Benziger et al., "First real time detection of ${ }^{7}$ Be solar neutrinos by Borexino," Physics Letters B, vol. 658, pp. 101-108, 2008.

[120] G. Alimonti, G. Anghloher, C. Arpesella et al., "Ultra-low background measurements in a large volume underground detector," Astroparticle Physics, vol. 8, pp. 141-157, 1998.
[121] G. Alimonti, C. Arpesella, H. Back et al., "Science and technology of Borexino: a real-time detector for low energy solar neutrinos," Astroparticle Physics, vol. 16, pp. 205-234, 2002.

[122] C. Arpesella, H. O. Back, M. Balata et al., "Measurements of extremely low radioactivity levels in BOREXINO," Astroparticle Physics, vol. 18, no. 1, pp. 1-25, 2002.

[123] A. Ianni, P. Lombardi, G. Ranucci, and O. Smirnov, "The measurements of 2200 ETL9351 type photomultipliers for the Borexino experiment with the photomultiplier testing facility at LNGS," Nuclear Instruments and Methods in Physics Research A, vol. 537, pp. 683-697, 2005.

[124] A. Brigatti, A. Ianni, P. Lombardi, G. Ranucci, and O. J. Smirnov, "The photomultiplier tube testing facility for the Borexino experiment at LNGS," Nuclear Instruments and Methods in Physics Research A, vol. 537, no. 3, pp. 521-536, 2005.

[125] L. Oberauer, C. Grieb, F. von Feilitzsch, and I. Manno, "Light concentrators for Borexino and CTF," Nuclear Instruments and Methods in Physics Research A, vol. 530, no. 3, pp. 453-462, 2004.

[126] Borexino Collaboration, "The Borexino detector at the Laboratori Nazionali del Gran Sasso," Nuclear Instruments and Methods in Physics Research A, vol. 600, pp. 568-593, 2009.

[127] H. Simgen and G. Zuzel, "Ultrapure gases-from the production plant to the laboratory," in Proceedings of the Topical Workshop on Low Radioactivity Techniques (LRT '06), P. Loaiza, Ed., vol. 897, pp. 45-50, American Institute of Physics, France, 2006.

[128] J. N. Bahcall, A. M. Serenelli, and S. Basu, "10,000 Standard solar models: a Monte Carlo simulation," The Astrophysical Journal Supplement Series, vol. 165, p. 400, 2006.

[129] G. Bellini, J. Benziger, D. Bick et al., "Precision measurement of the ${ }^{7}$ Be solar neutrino interaction rate in Borexino," Physical Review Letters, vol. 107, Article ID 141302, 5 pages, 2011.

[130] S. Basu, "Astronomical Society of the Pacific Conference Series," vol. 416, pp. 193, 2009.

[131] S. Davini, Measurement of the pep and CNO solar neutrino interaction rates in Borexino [Ph.D. thesis], University of Genova, 2012.

[132] C. Galbiati, A. Pocar, D. Franco, A. Ianni, L. Cadonati, and S. Schönert, "Cosmogenic ${ }^{11} \mathrm{C}$ production and sensitivity of organic scintillator detectors to pep and CNO neutrinos," Physical Review C, vol. 71, Article ID 055805, 11 pages, 2005.

[133] G. Bellini, J. Benziger, D. Bick et al., "Muon and cosmogenic neutron detection in Borexino," Journal of Instrumentation, vol. 6, Article ID P05005, 2011.

[134] M. Deutsch, Proposal for a Cosmic Ray Detection System for the Borexino Solar Neutrino Experiment, Massachusetts Institute of Technology, Cambridge, Mass, USA, 1996.

[135] H. Back and Borexino Collaboration, "CNO and pep neutrino spectroscopy in Borexino: measurement of the deep-underground production of cosmogenic ${ }^{11} \mathrm{C}$ in an organic liquid scintillator," Physical Review C, vol. 74, Article ID 045805, 6 pages, 2006.

[136] Y. Kino, The Journal of Nuclearand Radiochemical Sciences, vol. 1, pp. 63-68, 2000.

[137] D. Franco, G. Consolati, and D. Trezzi, "Positronium signature in organic liquid scintillators for neutrino experiments," Physical Review C, vol. 83, Article ID 015504, 6 pages, 2011.

[138] TMVA Users Guide, http://tmva.sourceforge.net/docu/ \%20TMVAUsersGuide.pdf.

[139] W. Maneschg, L. Baudis, R. Dressler et al., "Production and characterization of a custom-made ${ }^{228} \mathrm{Th}$ source with reduced 
neutron source strength for the Borexino experiment," Nuclear Instruments and Methods in Physics Research A, vol. 680, pp. 161167, 2012.

[140] L. Wolfenstein, "Neutrino oscillations in matter," Physical Review D, vol. 17, pp. 2369-2374, 1978.

[141] L. Wolfenstein, "Effect of matter on neutrino oscillations," in Proceedings of the Neutrino, pp. C3-C6, Purdue University, 1978.

[142] S. P. Mikheev and A. Y. Smirnov, "Resonant amplification of neutrino oscillations in matter and solar-neutrino spectroscopy," Soviet Journal of Nuclear Physics, vol. 42, pp. 913-917, 1985.

[143] S. P. Mikheev and A. Y. Smirnov, "Resonance enhancement of oscillations in matter and solar neutrino spectroscopy," Yadernaya Fizika, vol. 42, pp. 1441-1448, 1985.

[144] S. P. Mikheev and A. Y. Smirnov, "Resonant amplification of $v$ oscillations in matter and solar-neutrino spectroscopy," Nuovo Cimento C, vol. 9, no. 1, pp. 17-26, 1986.

[145] V. E. Zakharov and E. A. Kuznetsov, "Quasiclassical theory of three-dimensional wave collapse," Soviet Physics, JETP, vol. 64, no. 4, pp. 773-780, 1986, reprinted in: Solar neutrinos: the first thirty years, edited by: J. N. Bahcall.

[146] K. Nakamura and Particle Data Group, "Review of particle physics," Journal of Physics G, vol. 37, no. 7, Article ID 075021, 2010.

[147] B. Aharmim, S. N. Ahmed, A. E. Anthony et al., "Combined analysis of all three phases of solar neutrino data from the sudbury neutrino observatory," In press, http://arxiv.org/abs/ 1109.0763 .

[148] J. N. Bahcall, M. Kamionkowski, and A. Sirlin, "Solar neutrinos: radiative corrections in neutrino-electron scattering experiments," Physical Review D, vol. 51, no. 11, pp. 6146-6158, 1995.

[149] J. Erler and M. J. Ramsey-Musolf, "The Weak mixing angle at low energies," Physical Review D, vol. 72, Article ID 073003, 2005.

[150] J. N. Abdurashitov, V. N. Gavrin, V. V. Gorbachev et al., "Measurement of the solar neutrino capture rate with gallium metal. III. Results for the 2002-2007 data-taking period," Physical Review C, vol. 80, Article ID 015807, 16 pages, 2009.

[151] M. Altmann, M. Balatab, P. Belli et al., "Complete results for five years of GNO solar neutrino observations," Physics Letters B, vol. 616, pp. 174-190, 2005.

[152] F. Kaether, Datenanalyse der Sonnenneutrinoexperiments Gallex [Ph.D. thesis], Heidelberg, Germany, 2007.

[153] B. T. Cleveland, T. Daily, R. Davis Jr. et al., "Measurement of the solar electron neutrino flux with the homestake chlorine detector," The Astrophysical Journal, vol. 496, no. 1, p. 505, 1998.

[154] G. Bellini, J. Benziger, S. Bonetti et al., "Measurement of the solar ${ }^{8} \mathrm{~B}$ neutrino rate with a liquid scintillator target and $3 \mathrm{MeV}$ energy threshold in the Borexino detector," Physical Review D, vol. 82, Article ID 033006, 10 pages, 2010.

[155] K. Abe, N. Abgrall, Y. Ajima et al., "Indication of electron neutrino appearance from an accelerator-produced off-axis muon neutrino beam," Physical Review Letters, vol. 107, Article ID 041801, 8 pages, 2011.

[156] P. Adamson, D. J. Auty, D. S. Ayres et al., "Improved search for muon-neutrino to electron-neutrino oscillations in MINOS," Physical Review Letters, vol. 107, Article ID 181802, 5 pages, 2011.

[157] G. L. Fogli, E. Lisi, A. Marrone, A. Palazzo, and A. M. Rotunno, "Evidence of $\theta_{13}>0$ from global neutrino data analysis," Physical Review D, vol. 84, Article ID 053007, 7 pages, 2011.
[158] M. Apollonio, A. Baldini, C. Bemporad et al., "Search for neutrino oscillations on a long base-line at the $\mathrm{CHOOZ}$ nuclear power station," The European Physical Journal C, vol. 27, pp. 331374, 2003.

[159] Y. Abe, C. Aberle, T. Akiri et al., "Indication for the disappearance of reactor electron antineutrinos in the Double Chooz experiment," Physical Review Letters, vol. 108, Article ID 131801, 7 pages, 2012.

[160] C. Giunti and M. Laveder, "Effect of the reactor antineutrino anomaly on the first Double-Chooz results," Physical Review D, vol. 85, Article ID 031301, 4 pages, 2012.

[161] F. P. An, J. Z. Bai, A. B. Balantekin et al., "Observation of electron-antineutrino disappearance at daya bay," Physical Review Letters, vol. 108, Article ID 171803, 7 pages, 2012.

[162] J. K. Ahn, S. Chebotaryov, J. H. Choi et al., "Observation of reactor electron antineutrinos disappearance in the RENO experiment," Physical Review Letters, vol. 108, Article ID 191802, 6 pages, 2012.

[163] D. Dwyer, for the Daya-Bay Collaboration, talk at Neutrino, 2012.

[164] G. L. Fogli, E. Lisi, A. Marrone, D. Montanino, A. Palazzo, and A. M. Rotunno, "Global analysis of neutrino masses, mixings, and phases: entering the era of leptonic CP violation searches," Physical Review D, vol. 86, Article ID 013012, 10 pages, 2012.

[165] D. V. Forero, M. Tortola, and J. W. F. Valle, "Global status of neutrino oscillation parameters after Neutrino-2012," Physical Review D, vol. 86, Article ID 073012, 8 pages, 2012.

[166] T. Schwetz, "talk at NuTURN 2012," Workshop on Neutrino at the Turning Point, Laboratori Nazionali del Gran Sasso, Italy, 2012, http://agenda.infn.it/conferenceDisplay.py? confId=4722.

[167] "talk at what is NU ?" Workshop at the Galileo Galilei Institute, Florence, Italy, 2012, http://www.ggi.fi.infn.it/.

[168] A. Friedland, C. Lunardini, and C. Peña-Garay, "Solar neutrinos as probes of neutrino-matter interactions," Physics Letters B, vol. 594, pp. 347-354, 2004.

[169] P. C. de Holanda and A. Y. Smirnov, "Solar neutrino spectrum, sterile neutrinos, and additional radiation in the Universe," Physical Review D, vol. 83, Article ID 113011, 13 pages, 2011.

[170] M. Spiro and D. Vignaud, "Solar model independent neutrino oscillatiion signals in the forthcoming solar neutrino experiments?" Physics Letters B, vol. 242, pp. 279-284, 1990.

[171] J. N. Bahcall, "The luminosity constraint on solar neutrino fluxes," Physical Review C, vol. 65, Article ID 025801, 5 pages, 2002.

[172] M. C. González-García, M. Maltoni, and J. Salvado, "Updated global fit to three neutrino mixing: status of the hints of $\theta_{13}>0$," Journal of High Energy Physics, vol. 2010, no. 4, article 56, 2010.

[173] J. N. Bahcall and C. Peña-Garay, "A road map to solar neutrino fluxes, neutrino oscillation parameters, and tests for new physics," Journal of High Energy Physics, vol. 311, p. 4, 2003.

[174] A. Chavarria, "Solar neutrinos in 2011," In press, http://arxiv .org/abs/1201.6311.

[175] J. Boger, R. L. Hahna, J. K. Rowley et al., “The sudbury neutrino observatory," Nuclear Instruments and Methods in Physics Research A, vol. 449, pp. 172-207, 2000.

[176] S. Fukuda, Y. Fukuda, T. Hayakaw et al., "The Super-Kamiokande detector," Nuclear Instruments and Methods in Physics Research A, vol. 501, pp. 418-462, 2003.

[177] J. N. Abdurashitov, V. N. Gavrin, S. V. Girin et al., "Measurement of the solar neutrino capture rate with gallium metal," Physical Review C, vol. 60, Article ID 055801, 32 pages, 1999. 
[178] C. Kraus and S. J. M. Peetersa, "The rich neutrino programme of the SNO+ experiment," Progress in Particle and Nuclear Physics, vol. 64, pp. 273-277, 2010.

[179] F. L. Villante, A. Ianni, F. Lombardi, G. Pagliaroli, and F. Vissani, "A step toward CNO solar neutrino detection in liquid scintillators," Physics Letters B, vol. 701, no. 3, pp. 336-341, 2011.

[180] D. N. McKinsey and K. J. Coakley, "Neutrino detection with CLEAN," Astroparticle Physics, vol. 22, no. 5-6, pp. 355-368, 2005.

[181] E. Aprile and T. Doke, "Liquid xenon detectors for particle physics and astrophysics," Reviews of Modern Physics, vol. 82, pp. 2053-2097, 2010.

[182] A. Baldini, "Research Proposal to INFN, The MEG experiment, search for the $u \rightarrow e \gamma$ decay at PSI," 2002.

[183] S. Moriyama, "Status of XMASS experiment," PoS IDM, vol. 2011, p. 57, 2010.

[184] K. Kobayashi, "XMASS experiment, talk given at the TeV Particle Astrophysics 2010," Paris, France, July 2010.

[185] L. Baudis, "DARWIN: dark matter WIMP search with noble liquids," Journal of Physics, vol. 375, part 1, Article ID 012028, 2012.

[186] M. Wurm, J. F. Beacomc, L. B. Bezrukov et al., "The nextgeneration liquid-scintillator neutrino observatory LENA," Astroparticle Physics, vol. 35, pp. 685-732, 2012.

[187] H. O. Backa, M. Balata, A. de Bari et al., "Study of phenylxylylethane (PXE) as scintillator for low energy neutrino experiments," Nuclear Instruments and Methods in Physics Research A, vol. 585 , no. 12 , pp. 48-60, 2008.

[188] C. Arpesella, H. O. Back, M. Balata et al., "Direct measurement of the ${ }^{7}$ Be solar neutrino flux with 192 days of borexino data," Physical Review Letters, vol. 101, Article ID 091302, 6 pages, 2008.

[189] M. Wurm, Cosmic background discrimination for the rare neutrino event search in Borexino and LENA [Ph.D. thesis], Technische Universität München, 2009.

[190] D. D'Angelo, Towards the detection of low energy solar neutrinos in BOREXino: data readout, data reconstruction and background identification [Ph.D. thesis], Technische Universität München, 2006.

[191] A. Ianni, D. Montanino, and F. L. Villante, "How to observe ${ }^{8}$ B solar neutrinos in liquid scintillator detectors," Physics Letters B, vol. 627, pp. 38-48, 2005.

[192] R. S. Raghavan, "Inverse $\beta$ decay of ${ }^{115} \mathrm{In} \rightarrow{ }^{115} \mathrm{Sn} *:$ a new possibility for detecting solar neutrinos from the Proton-Proton reaction," Physical Review Letters, vol. 37, pp. 259-262, 1976.

[193] R. S. Raghavan, "Discovery potential of low energy solar neutrino experiments," Notes for APSSAWG, March 2004.

[194] H. Antia and S. Basu, "The discrepancy between solar abundances and helioseismology," The Astrophysical Journal Letters, vol. 620, p. L129, 2005.

[195] J. N. Bahcall, S. Basu, and A. M. Serenelli, "What is the neon abundance of the sun?" Astrophysical Journal Letters, vol. 631, no. 2, pp. 1281-1285, 2005.

[196] J. A. Guzik, L. S. Watson, and A. N. Cox, "Can enhanced diffusion improve helioseismic agreement for solar models with revised abundances?" Astrophysical Journal, vol. 627, no. 2, pp. 1049-1056, 2005.

[197] M. Castro, S. Vauclair, and P. Richard, "Low abundances of heavy elements in the solar outer layers: comparisons of solar models with helioseismic inversions," Astronomy \& Astrophysics, vol. 463, pp. 755-758, 2007.
[198] J. Christensen-Dalsgaard, M. P. di Mauro, G. Houdex, and F. Pijpers, "On the opacity change required to compensate for the revised solar composition," Astronomy \& Astrophysics, vol. 494, pp. 205-208, 2009.

[199] A. M. Serenelli, "New results on standard solar models," Astrophysics and Space Science, vol. 328, pp. 13-21, 2010.

[200] F. L. Villante and B. Ricci, "Linear solar models," Astrophysical Journal Letters, vol. 714, no. 1, pp. 944-959, 2010.

[201] F. L. Villante, "Constraints on the opacity profile of the sun from helioseismic observables and solar neutrino flux measurements," The Astrophysical Journal, vol. 724, p. 98, 2010.

[202] J. Barranco, O. G. Miranda, C. A. Moura, and J. W. F. Valle, "Constraining nonstandard neutrino-electron interactions," Physical Review D, vol. 77, no. 9, Article ID 093014, 10 pages, 2008.

[203] H. A. Bethe, "Energy production in stars," Physical Review, vol. 55, pp. 434-456, 1939.

[204] A. A. Thoul, J. N. Bahcall, and A. Loeb, "Element diffusion in the solar interior," Astrophysical Journal Letters, vol. 421, no. 2, pp. 828-842, 1994.

[205] F. Delahaye, M. H. Pinsonneault, L. Pinsonneault, and C. J. Zeippen, "Helioseismic constraintson the solar $\mathrm{Ne} / \mathrm{O}$ ratio and heavy element abundances," submitted, http://arxiv.org/ abs/1005.0423.

[206] J. Meléndez, M. Asplund, B. Gustafsson, and D. Yong, "The peculiar solar composition and its possible relation to planet formation," The Astrophysical Journal Letters, vol. 704, p. L66, 2009.

[207] I. Ramírez, M. Asplund, P. Baumann, J. Meléndez, and T. Bensby, "A possible signature of terrestrial planet formation in the chemical composition of solar analogs," Astronomy \& Astrophysics, vol. 512, p. 33, 2010. 

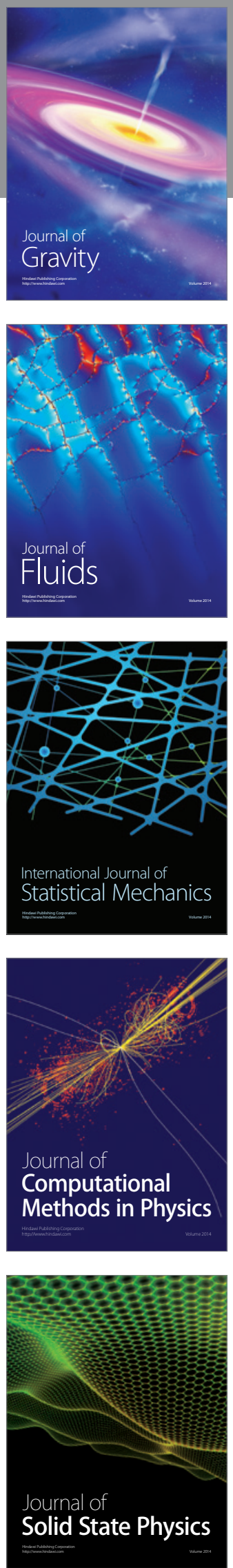

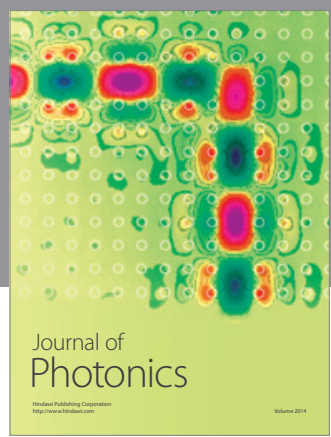

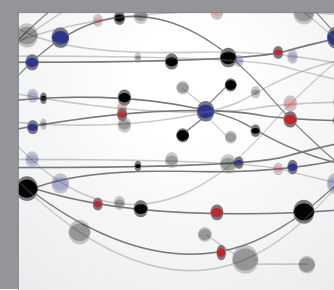

The Scientific World Journal

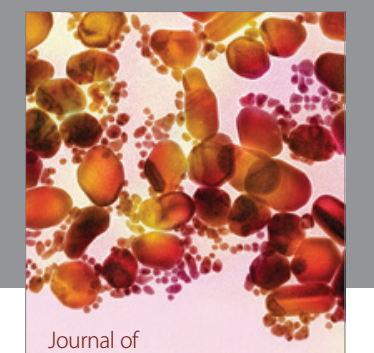

Soft Matter
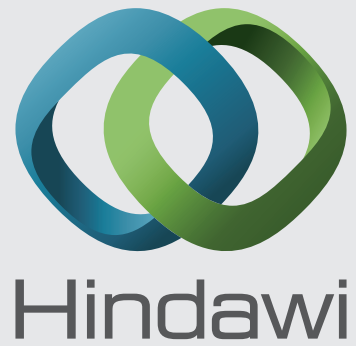

Submit your manuscripts at

http://www.hindawi.com
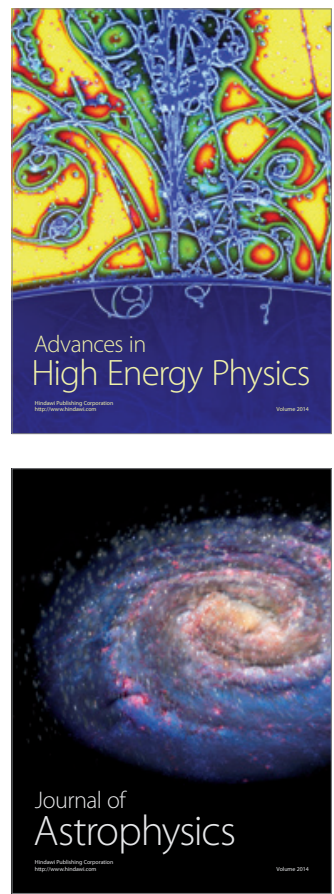
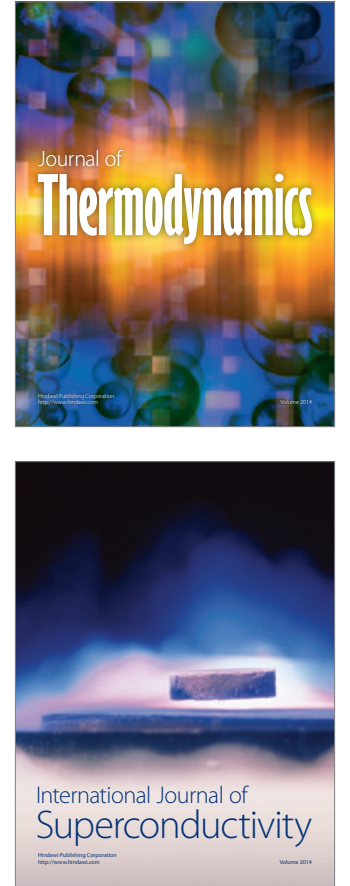
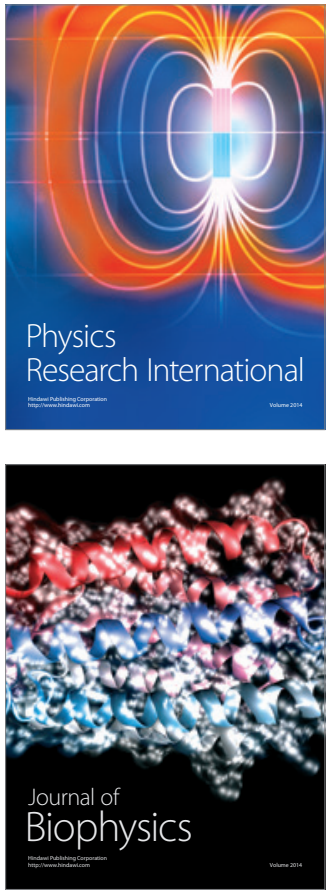
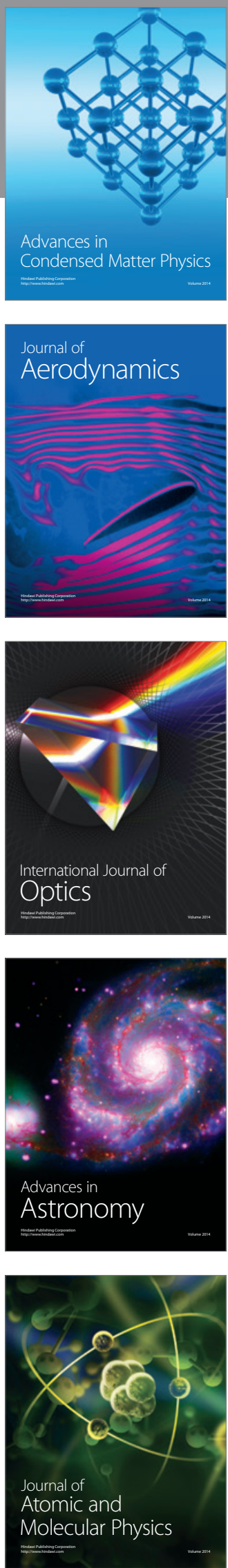